Portland State University

PDXScholar

Fall 12-10-2014

\title{
The Role of Mate Preference and Personality on Reproductive Performance in an Ex-Situ Conservation Breeding Program for the Giant Panda (Ailuropoda melanoleuca)
}

Meghan S. Martin

Portland State University

Follow this and additional works at: https://pdxscholar.library.pdx.edu/open_access_etds

Part of the Behavior and Ethology Commons, and the Other Animal Sciences Commons Let us know how access to this document benefits you.

\section{Recommended Citation}

Martin, Meghan S., "The Role of Mate Preference and Personality on Reproductive Performance in an ExSitu Conservation Breeding Program for the Giant Panda (Ailuropoda melanoleuca)" (2014). Dissertations and Theses. Paper 2096.

https://doi.org/10.15760/etd.2094

This Dissertation is brought to you for free and open access. It has been accepted for inclusion in Dissertations and Theses by an authorized administrator of PDXScholar. Please contact us if we can make this document more accessible: pdxscholar@pdx.edu. 
The Role of Mate Preference and Personality

on Reproductive Performance in an Ex-Situ Conservation Breeding Program

for the Giant Panda (Ailuropoda melanoleuca)

by

Meghan S. Martin

A dissertation submitted in partial fulfillment of the requirements for the degree of

Doctor of Philosophy

in

Biology

Dissertation Committee:

Deborah Duffield, Chair

David Shepherdson, Co-Chair

Ronald Swaisgood

Deborah Lutterschmidt

Randy Zelick

Natalie Vasey

Portland State University

2014 
(C) 2014 Meghan S. Martin 


\begin{abstract}
Successful captive-breeding and re-introduction programs must have the ability to breed a surplus of genetically suitable animals for release into the wild. Unfortunately, many individuals in captive breeding programs often do not reproduce even when they are apparently healthy and presented with genetically appropriate mates. Mate preference can affect multiple parameters of reproductive sperformance, including mating success, offspring production, survival, and fecundity. We investigated the role of mate preference and personality on the reproductive performance of male and female giant pandas (Ailuropoda melanoleuca) as measured by intromission success and litter production. We conducted these studies on giant pandas at the China Conservation and Research Center for the Giant Panda (Bifengxia, Sichuan, China.) from February-May 2011, 2012, and 2013.
\end{abstract}

In Chapter 2, we compared reproductive performance between individuals mated with preferred and nonpreferred males of the focal female (female preference), the focal male (male preference), and mutual mate preference perspective. There were four types of reproductive pairings in our mutual mate preference analyses: females and males that preferred each other (P-P), females that preferred the male but the male did not prefer the female (P-NP), females that did not prefer the male but the male preferred the female (NP-P), and females and males that did not prefer each other (NP-NP). Pairing giant pandas with a preferred partner increased the probability of intromission success and producing a cub. Of the 25 females mated to a preferred partner, $72 \%$ had successful intromission, $64 \%$ produced cubs, and $52 \%$ reared their own cubs versus $31 \%, 12 \%, 12 \%$ for females mated to their nonpreferred partner $(\mathrm{N}=16)$. Male giant panda mate 
preference showed similar results. Of the 24 males that were mated with their preferred females, $75 \%$ had successful intromission, $77.8 \%$ produced cubs, and $92.9 \%$ produced cubs that were maternally reared versus $31 \%, 60 \%, 66.7 \%$ for males mated with their nonpreferred females $(\mathrm{N}=16)$. There was a statistically significant difference in intromission success and cub production for P-P pairings versus NP-NP pairings. Of the twelve P-P mate dyads $83 \%$ had successful intromission, $90 \%$ produced cubs, and $88.9 \%$ had mothers rear their cubs. Of the four P-NP mate dyads 50\% had successful intromission, $50 \%$ produced cubs, and $100 \%$ had the mother rear their cub. Of the seven NP-P mate dyads $57 \%$ had successful intromission, $50 \%$ produced cubs, and $100 \%$ had the mothers rear their cubs. Of the three NP-NP mate dyads $0 \%$ had successful intromission resulting in $0 \%$ producing cubs and having mother-rear cubs. In addition, we took into account various life and breeding history factors that may have affected reproductive performance such as birth location, rearing, previous sexual history with the opposite sex and familiarity status directly prior to breeding. Mate pairings that were familiar with each other as measured by successfully producing a cub in the past, had increased intromission success. Females mated to heavier males had more intromissions and produced more cubs than females mated to smaller males.

In Chapter 3 and 4, we investigated the effect of personality on reproductive performance measurements. Recently, personality of individual animals has been implicated in the failure or success of captive management and breeding of threatened and endangered species. Measurements of personality included novel object tests (where behaviors of individual giant pandas were scored toward a novel object for one hour) and keeper surveys (where the primary keeper rated giant pandas on 23 adjectives describing 
personality); both measures significantly correlated with variation in long-term and specific mating dyad reproductive performance.

In Chapter 3 we evaluated the effect of personality on long-term reproductive performance. Our results show that for both male and female giant pandas, 'fearful' individuals had lower natural mating success and cub production. In addition, 'aggressive' female giant pandas had increased natural mating success but decreased cub production. Males did not show these relationships between 'aggressive' personality types and reproductive performance. Female giant pandas that were labeled as 'aggressive' had higher frequencies of positive mating behaviors but 'fearful of conspecifics' females had lower frequencies. Males labeled 'fearful of conspecifics' and with high 'playful-clever' component scores had a lower frequency of positive mating behaviors.

In Chapter 4 we further investigated the effects of personality within a specific mating dyad. Recent studies suggest that mate compatibility within personality types can improve reproductive performance measurements such as mating success, offspring survivorship, and offspring quality. We investigated the effect of personality compatibility on reproductive performance through analyzing mate dyad similarity in personality (similar versus dissimilar), specific personality combinations, and relative personality scores (male scores higher or lower than female scores). The specific personality combinations were: females and males that were both low on the personality component $\left(\mathrm{L} \_\mathrm{L}\right)$, females were low but males were high $\left(\mathrm{L} \_\mathrm{H}\right)$, females were high but males were low $\left(\mathrm{H} \_\mathrm{L}\right)$, and females and males were both high $\left(\mathrm{H} \_\mathrm{H}\right)$. Our results suggest that giant pandas appear to be assortatively mating based on aggressive, fearful, and 
stereotypical-excitable personality traits. Mate dyads that were dissimilar on the stereotypical-excitable component score were more likely to have successful intromission than pairs that were similar. L_H mate dyads on the aggressive and stereotypicalexcitable personality traits had increased intromission success and cub production than all other possible personality combinations. L_L on fearfulness had higher intromission success but not higher cub production than all other possible personality combinations. Mate dyads composed of males ranked high on the aggressive component relative to the female resulted in higher reproductive performance and cub production.

In captive breeding programs, mates are traditionally selected solely on the basis of genetic parameters to minimize loss of genetic diversity and inbreeding coefficients. Our results suggest that integrating genetic with behavioral measures of preference, breeding history factors, and personality would increase the reproductive output of the giant panda captive-breeding program. We stress the importance of considering husbandry practices to accommodate personality traits and ultimately increase reproductive performance in the captive population while simultaneously maintaining both genetic and behavioral diversity. 


\section{ACKNOWLEDGMENTS}

First and foremost I would like to thank my beautiful family without whose support, love, and humor I would not have survived this process. My talented husband, Nate Wintle, made it possible for our family to stay together while abroad in China and for this I will be forever in his debt. My charming and bright daughter made transcending cultural barriers infinitely easier and the memories she gave to her parents during this process will be forever cherished.

I have many individuals and organizations to thank for their support during this project. Without the support of Portland State University, the Oregon Zoo, San Diego Zoo, and Chinese Conservation and Research Center for the Giant Panda (CCRCGP), the completion of this dissertation would not have been possible. I thank my advisors, Debbie Duffield and David Shepherdson, and co-advisor, Ron Swaisgood, for cultivating this collaboration among institutions and helping me carry out this important research away from home. I greatly appreciate the cooperation of the keepers and staff of the CCRCGP and of Directors Li Desheng, Zhang Hemin, Zhou Xiaoping, Zhang Gui Quan,

Huang Yan, Tang Chunxiang. In addition, I need to thank Wang Cheng Dong, Wu Hong Lin, Huang Jin Yan, Huang Zhi, Luo Bo, and Wei Ming for their efforts in collecting vital parts of this research and mentoring me in giant panda reproductive behavior. Li Ruiqiang was my counterpart at the CCRCGP during my absence and greatly helped in organizing efforts by his team specifically Sophie for compiling last minute data. I would like to thank all my committee members, Debbie Duffield, David Shepherdson, Ron Swaisgood, Deborah Lutterschmidt, Randy Zelick, and Natalie Vasey 
for their support and understanding throughout the dissertation process. Thanks to Deborah Lutterschmidt for her keen insight on hormonal experimental design (even when it ultimately resulted in no product) and understanding of my completely horrific editing abilities. Randy Zelick for encouraging me to hone my skills through invited guest lectures and insightful comments on my proposal defense. Natalie Vasey for encouraging me to connect with past projects and explore new ones. Not enough thanks can be directed to Debbie Duffield whose undying patience and tolerance for terrible first drafts was crucial to shaping this final product and whose continuous support has fostered me through both my Masters degree and my PhD. Special thanks goes to Ron Swaisgood who responded to texts, emails, and skype calls at all hours to advise my efforts despite the time difference between the US and China and who took a chance on passing his lifelong contacts at the CCRCGP to an 'unknown' student. Finally thanks goes to David Shepherdson who has encouraged and mentored me through my sometimes budding, sometimes stumbling, career as a conservation biologists and without whose support I would not be the scientist I am today. I owe you all my most humble and heartfelt gratitude.

I could not have gotten as far as I have without the support of my parents, Debbie and Steve Martin, or my siblings, Ashley, Aaron and Brynn - thanks for everything you have done over the years! They encouraged me to pursue all of my goals, sometimes without fully understanding them. They instilled in me a love for animals and an analytical (yet tolerant) approach to life, for which I will be forever grateful. 
I cannot forget my numerous friends and colleagues who graded, critiqued, advised and let me complain to them about this thesis; Althea Hayden, Diana Dishman, Victoria Alla, Jan Irvahn, and Sharon Glaeser.

Last but not least, I would like to thank the foundations that financially supported this work and recognized its importance; the Oregon Zoo's Future for Wildlife grant, Animal Behavior's Student Research Grant, PDXWildlife, and the Forbes Lea Foundation. You all made this work possible. 


\section{TABLE OF CONTENTS}

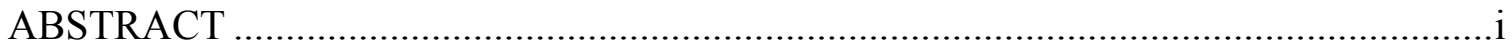

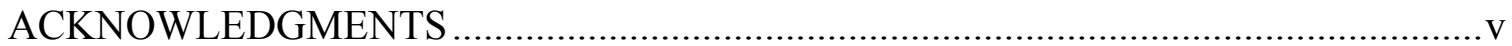

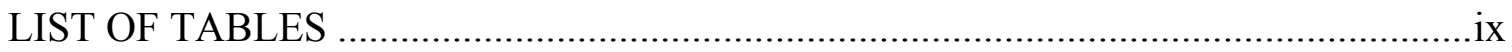

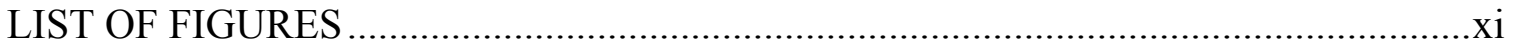

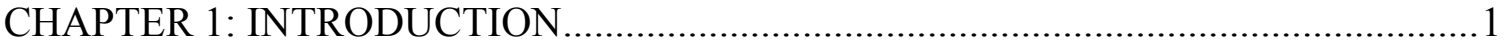

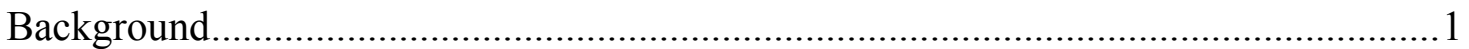

CHAPTER 2: DOES CHOICE MATTER? THE ROLE OF MATE PREFERENCE ON

REPRODUCTIVE PERFORMANCE OF MALE AND FEMALE GIANT PANDAS...13

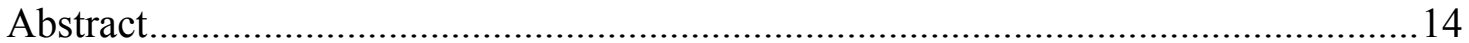

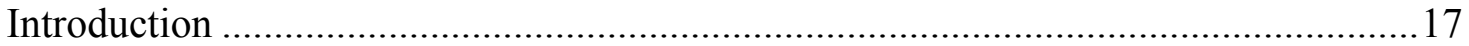

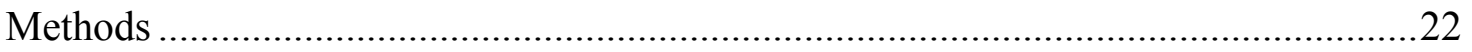

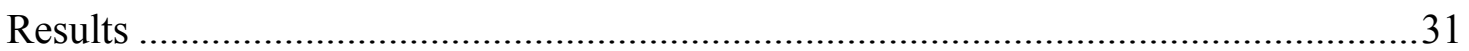

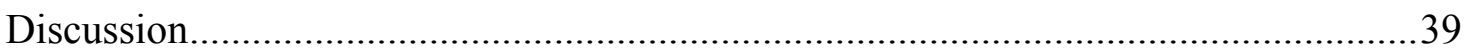

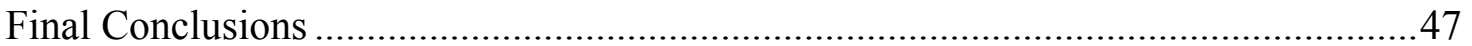

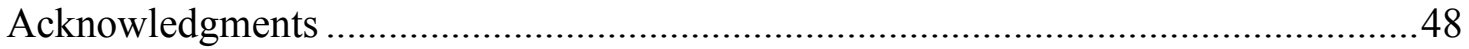

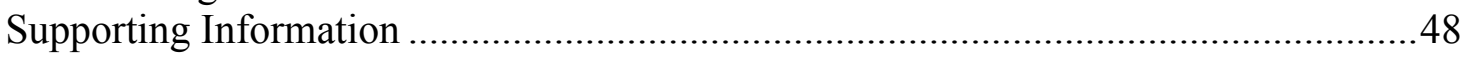

CHAPTER 3: PERSONALITY AS A PREDICTOR OF LONG-TERM

REPRODUCTIVE PERFORMANCE IN CAPTIVE GIANT PANDAS..........................58

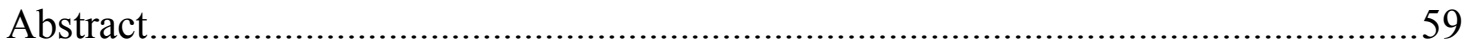

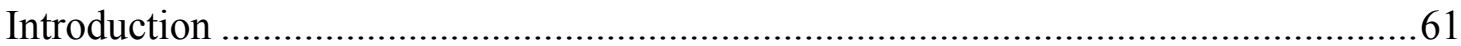

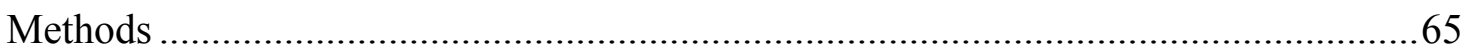

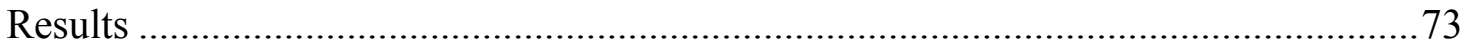

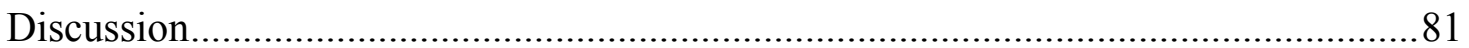

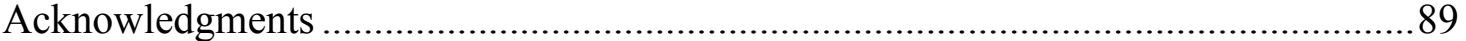

CHAPTER 4: DO OPPOSITES ATTRACT? PERSONALITY DISSIMILARITY IN BREEDING PAIRS OF CAPTIVE GIANT PANDAS INCREASE REPRODUCTIVE

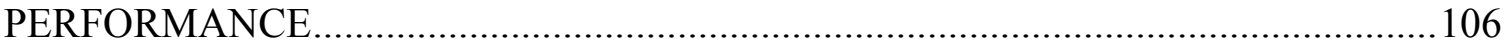

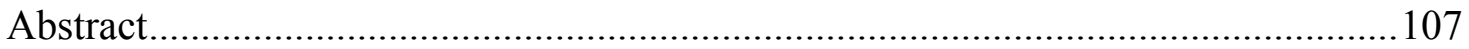

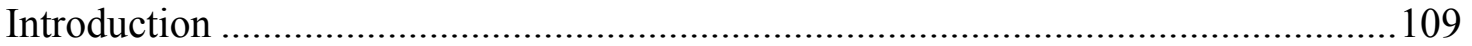

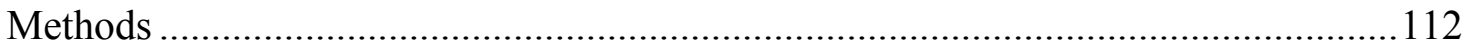

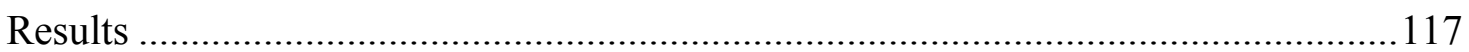

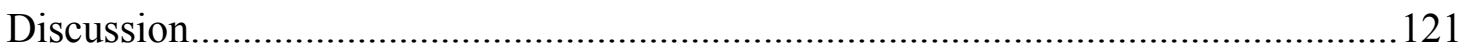

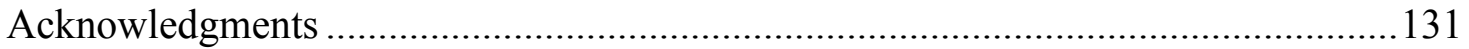

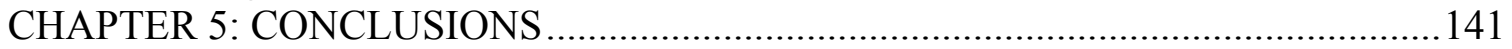

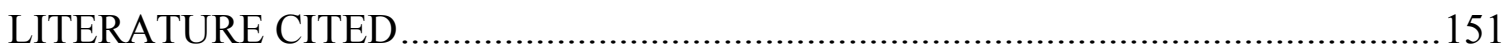

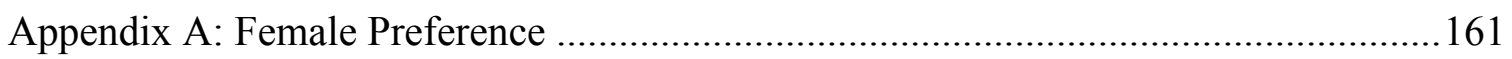

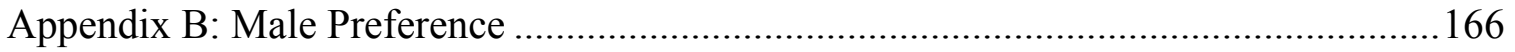

Appendix C: Personality and Long-term Reproductive performance ..............................170

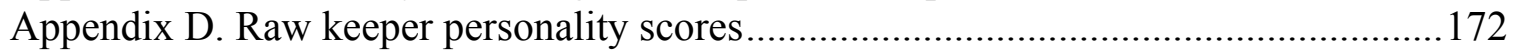




\section{LIST OF TABLES}

Table 2.1. Measures of reproductive performance for mate pairings of female giant pandas.

Table 2.2. Measures of reproductive performance for mate pairings of male giant pandas.

Table 2.3. Components of reproductive performance by mating type in giant pandas.....51

Table 3.1. Behavioral definitions of adjectives used for questionnaire ratings................90

Table 3.2. Behavioral definitions of adjectives used for questionnaire ratings................91

Table 3.3. Four major components of individual behavior variation in captive giant pandas derived from combined score results of keeper questionnaires

Table 3.4. Four major components of individual behavior variation in captive giant pandas derived from behavioral scores on ball novel object test

Table 3.5. Means and ranges for behavioral responses of giant pandas to novel objects .94

Table 3.6. Spearman rank-order correlation coefficients between quantitative behavioral responses of giant pandas to the rubber ball novel object and scores obtained from keeper questionnaires and resulting component scores.

Table 3.7. Mean and standard deviation of component scores of individual giant pandas grouped by breeding status, gender, rearing type, and provenance.

Table 3.8. Spearman rank-order correlation coefficients between average mating behaviors of giant pandas to scores obtained from keeper questionnaires.

Table 4.1. Means and ranges for female and male giant pandas ranked 'low' and 'high' based on principal components obtained from keeper ratings of giant panda personalities.

Table 4.2. Statistical analyses of reproductive performance for mate dyads of giant pandas grouped by personality similarity, combinations of personalities, and relative personalities base on component (PCA) scores for aggressive, playful-clever, fearful, and stereotypical-excitable.

Table 4.3. Values and measures of reproductive performance for mate dyads of giant pandas grouped by personality combinations based on principal component (PCA) scores for aggressive, playful-clever, fearful, and stereotypical-excitable. 
Table 4.4. Significant findings of increased reproductive performance for mate dyads of giant pandas grouped by personality similarity, combinations of personalities, and relative personalities base on component (PCA) scores for aggressive, playfulclever, fearful, and stereotypical-excitable. 


\section{LIST OF FIGURES}

Figure 2.1. Percentage of mate dyads for females' preferred and nonpreferred mates grouped by intromission success, cub production, and maternal rearing for giant pandas.

Figure 2.2. Percentage of mate dyads for females' mated with males that had previously sired one of their cubs and males that had not grouped by intromission success, cub production, and maternal rearing for giant pandas.

Figure 2.3. Mean mass of a) males introduced to females that had successful intromission and females that did not have successful intromission, b) males introduced to females that produced cubs and females that did not produce cubs, c) males introduced to females that reared their cubs and females that did not rear their cubs.

Figure 2.4. Percentage of mate dyads for males' preferred and nonpreferred mates grouped by intromission success, cub production, and maternal rearing for giant pandas.

Figure 2.5. Percentage of mate dyads for males' mated with females that had previously sired one of their cubs and females that had not grouped by intromission success, cub production, and maternal rearing for giant pandas.

Figure 2.6. Percentage of mate dyads for different combinations of male and female preference; male does not nonpreferred and female is nonpreferred (NP-NP), female prefers male but male does not prefer female (P-NP), female does not prefer male but male prefers female (NP-P), and both animals prefer each other.

Figure 3.1. Mean age of breeder and non-breeder giant pandas at the China Conservation and Research Center for the Giant Panda in 2012 and 2013. .98

Figure 3.2. Mean fearful component scores of breeder and non-breeder giant pandas....99

Figure 3.3. Fearful Component as a function of age in giant pandas..... 100

Figure 3.4a. Average keeper scores on 'aggressive toward conspecifics' questionnaire factor for female giant pandas that produced cubs and did not produce cubs

Figure 3.4b. Average keeper scores on 'curious' questionnaire factor for female giant pandas that produced cubs and did not produce cubs 
Figure 3.4c. Average keeper scores on 'fearful of people' questionnaire factor for female giant pandas that produced cubs and did not produce cubs.

Figure 3.4d. Average scores on fearful component (III) for female giant pandas that produced cubs and did not produce cubs

Figure 3.4e. Average scores on aggressive component (I) for female giant pandas that produced cubs and did not produce cubs

Figure 4.1. The percent of mate dyads resulting in a) successful intromission and b) cub production for mate pairings composed of females and males ranked similar to each other than females and males ranked dissimilar to each other on the stereotypical PCA component scores 136

Figure 4.2. The percent of mate dyads resulting in a) successful intromission and b) cub production for mate pairings of females and males that were both ranked low on aggressive component scores (L_L), of females that were ranked low and males that were ranked high $\left(\mathrm{L}_{-} \mathrm{H}\right)$, of females that were ranked high and males that were ranked low $\left(\mathrm{H} \_\mathrm{L}\right)$, and of females and males that were both ranked high $\left(\mathrm{H} \_\mathrm{H}\right) . .137$

Figure 4.3. The percent of mate dyads resulting in a) successful intromission and b) cub production for mate pairings composed of females and males that were both ranked low on fearful component scores ( $\left.\mathrm{L}_{-} \mathrm{L}\right)$, of females that were ranked low and males that were ranked high $\left(\mathrm{L}_{-} \mathrm{H}\right)$, of females that were ranked high and males that were ranked low $\left(\mathrm{H} \_\mathrm{L}\right)$, and of females and males that were both ranked high $\left(\mathrm{H} \_\mathrm{H}\right) . .138$

Figure 4.4. The percent of mate dyads resulting in a) successful intromission and b) cub production for mate pairings of females and males that were both ranked low on stereotypical-excitable component scores $\left(L_{-} L\right.$ ) , of females that were ranked low and males that were ranked high $\left(\mathrm{L}_{-} \mathrm{H}\right)$, of females that were ranked high and males that were ranked low $\left(\mathrm{H}_{-} \mathrm{L}\right)$, and of females and males that were both ranked high ( 139

Figure 4.5. The percent of mate dyads resulting in a) successful intromission and b) cub production for mate pairings composed of females mated to males ranked lower than them on aggressive component scores and females mated to males ranked higher than them on aggressive component scores 


\section{CHAPTER 1: INTRODUCTION}

\section{Background}

Captive breeding programs are considered essential for the propagation and health of endangered species. To be successful, captive breeding programs require that the majority of the reproductively mature adults in the population reproduce successfully. However, in practice many individuals fail to reproduce even when presumably fully reproductive and healthy. Historically, captive breeding programs have focused primarily on pairing animals based on the future genetic health of the population (Snyder et al. 1996a), while largely ignoring the potential importance of mate choice and mate competition (Swaisgood \& Schulte 2010). Current captive breeding methods have been largely unsuccessful at maintaining and/or growing populations of rare mammals; as of 2013, only 37 (6\%) out of 595 species in captivity were considered self-sustaining ( $A Z A$ Sustainable Zoo \& Aquarium Populations Report 2013).

Conservation breeding programs are an important tool in the recovery of the iconic endangered species, the giant panda (Ailuropoda melanoleuca). Like other endangered species, giant pandas have been notoriously reluctant to breed in captivity (Swaisgood et al. 2006; Swaisgood et al. 2003b). In 1996, Chinese researchers estimated that only $39 \%$ of captive female pandas produced offspring, $80 \%$ failed to display normal behavioral estrus, and only six living males in captivity had ever mated naturally (Lindburg et al. 1998). There are two main facilities that breed giant pandas in China, the China Conservation and Research Center for Giant Pandas in Wolong (CCRCGP, now relocated to Bifengxia following the 2008 earthquake that destroyed the original facility) 
and the Chengdu center located near the city center in Chengdu. The CCRCGP has long been China's most successful breeding facility. By 2005, the CCRCGP captive population had tripled from about 25 to more than 70 individuals (Swaisgood et al. $2003 \mathrm{~b}$ ), and the current population is in excess of 80 animals. The success of the Wolong breeding center has long been anecdotally attributed to the implementation of a natural mating program. This program involves housing animals in large natural outdoor enclosures prior to the breeding season, monitoring estrus closely to accurately predict when animals should be introduced for breeding, and familiarization of multiple males to a female prior to breeding through close proximity housing. Although these management changes are known to affect sexual motivation, the specific outcomes for reproduction are unknown due to lack of controlled experiments on reproductive performance in giant pandas.

One prominent candidate for a causal relationship between mating conditions and reproductive performance is mate preference. In most captive breeding situations breeding pairs are decided based on minimizing genetic relatedness rather than the animal's own mating preference (Asa et al. 2011). Many zoo and breeding facility programs give animals a single option for a mate and the success or failure hangs on the outcome of that one pairing. However, recent studies have shown that giving an animal a choice between two or more opposite sex conspecifics can drastically increase the reproductive performance of the choosing individual (Drickamer et al. 2003; Martin \& Shepherdson 2012). The objective of the proposed research is to determine the effect of mate preference and personality on several parameters important for reproductive performance in giant pandas. 


\section{Giant Panda Ecology}

The giant panda is a bear native to central-western and southwestern China. Historically, the giant panda could be found in nearly all regions of China, but its current range has been drastically reduced to portions of six isolated mountain ranges (Minshan, Qinling, Qionglai, Liangshan, Daxiangling, and Xiaoxiangling) in Gansu, Shaanxi and Sichuan Provinces. What is known about the giant panda's behavior in the wild is based on three long-term field studies in the Wolong Nature Reserve (Schaller et al. 1985), the Qinling mountains (Lu et al. 1994; Zhi et al. 2000), and recently in Foping Nature Reserve (Nie et al. 2012a; Nie et al. 2012b; Nie et al. 2012c). Giant pandas are solitary as adults, avoiding direct contact with conspecifics outside of the breeding season, and occupy home ranges that overlap extensively with conspecifics of both sexes (Swaisgood et al. 2003b). During the non-mating season pandas vocalize infrequently but during the mating season males will communicate long-distance via "song medleys" (Charlton et al. 2009). In contrast, throughout the year pandas rely heavily on chemical communication and will create sites used to deposit urine and scent from anogenital glands that are visited by pandas from overlapping ranges (Schaller et al. 1985; Swaisgood et al. 2003b).

In the wild, the mating season for giant pandas begins in February and continues through the end of April (Nie et al. 2012a; Nie et al. 2012b; Nie et al. 2012c). In captivity, the breeding season can extend into May, however it is rare for females to go into estrus this late (pers. obs). Females experience a single estrus each spring where they are fertile for 1-3 days (Kleiman 1983; Swaisgood et al. 2003b). Males display seasonal rut-like behavior and physiological changes (Aitken-Palmer et al. 2012; 
Kleiman 1983). Several males will locate a female in estrus and aggressively compete for a mating opportunity while the female panda climbs a nearby tree, descending once the male victor has been established (Schaller et al. 1985).

Giant pandas exhibit delayed implantation, where the fertilized embryo's development is arrested and uterine implantation is delayed (Monfort et al. 1989; Zhang et al. 2009), which can last anywhere from a few weeks to months, thus, the gestation period ranges from 97 to 161 days (Zhang et al. 2009). At birth, panda cubs weigh between 90 and 130g and are the most altricial of all eutherian mammals (Swaisgood et al. 2003b). Giant panda mothers usually give birth to singletons but twins are common in captivity and triplets were recorded recently, though this is extremely rare (Geggel 2014). Infant panda cubs are born with their eyes closed and are entirely reliant on their mothers for care. Cubs will nurse anywhere from 6 to 12 times per day in their first two weeks of life (Peng et al. 2001). The mother will not leave the cub for at least 15 days because it is critically important for her to regulate the cub's temperature, feeding, and defecation (Peng et al. 2001; Swaisgood et al. 2003b). The cub is weaned and separated from its mother at 18-24 months of age in the wild (Swaisgood et al. 2003b), however, in captivity it is typical to separate the mother and baby at 6 months in order to breed females yearly.

\section{Pre-mating and Mating Behaviors}

The giant panda has been the subject of captive and field studies for over 40 years. The courtship behavior of the giant panda has been measured via well-developed ethograms and has distinct patterns leading up to estrus for both males and females 
(Swaisgood et al. 2003b). Such established behavioral metrics make it possible to quantitatively compare responses among mate preference trials.

Swaisgood et al. (2003b) characterized detailed reports on male-female interactions throughout the female estrous cycle. These studies reported that behavior of the female giant panda changes dramatically during estrus. Prior to estrus, females generally ignore male giant pandas or respond aggressively towards them. However, as the female approaches the early stages of estrus she shows more interest in males, approaching frequently and emitting a combination of affiliative (bleat, chirp) and aggressive (moan, bark, growl) vocalizations. At peak estrus, the female increases her behaviors toward neighboring males with frequent and emphatic chirping and bleating, presentation of her hindquarters, and adopting the lordosis posture with her tail up. The onset and termination of behaviors seen during estrus in the female giant panda are shown in Figure 1. The male giant pandas increase their affiliative and sexual behavior towards the female as she approaches the fertile period of estrus. The presence of these pre-mating behaviors has been used extensively by managers to determine whether a potential mate pair is behaviorally compatible for a mating introduction. However, to my knowledge, no previous study has investigated which pre-mating behaviors correspond with successful mating ${ }^{1}$ and eventual reproductive performance ${ }^{2}$.

\footnotetext{
${ }^{1}$ Successful mating is defined throughout this paper as intromission.

${ }^{2}$ Reproductive performance means producing a cub and raising the cub.
} 


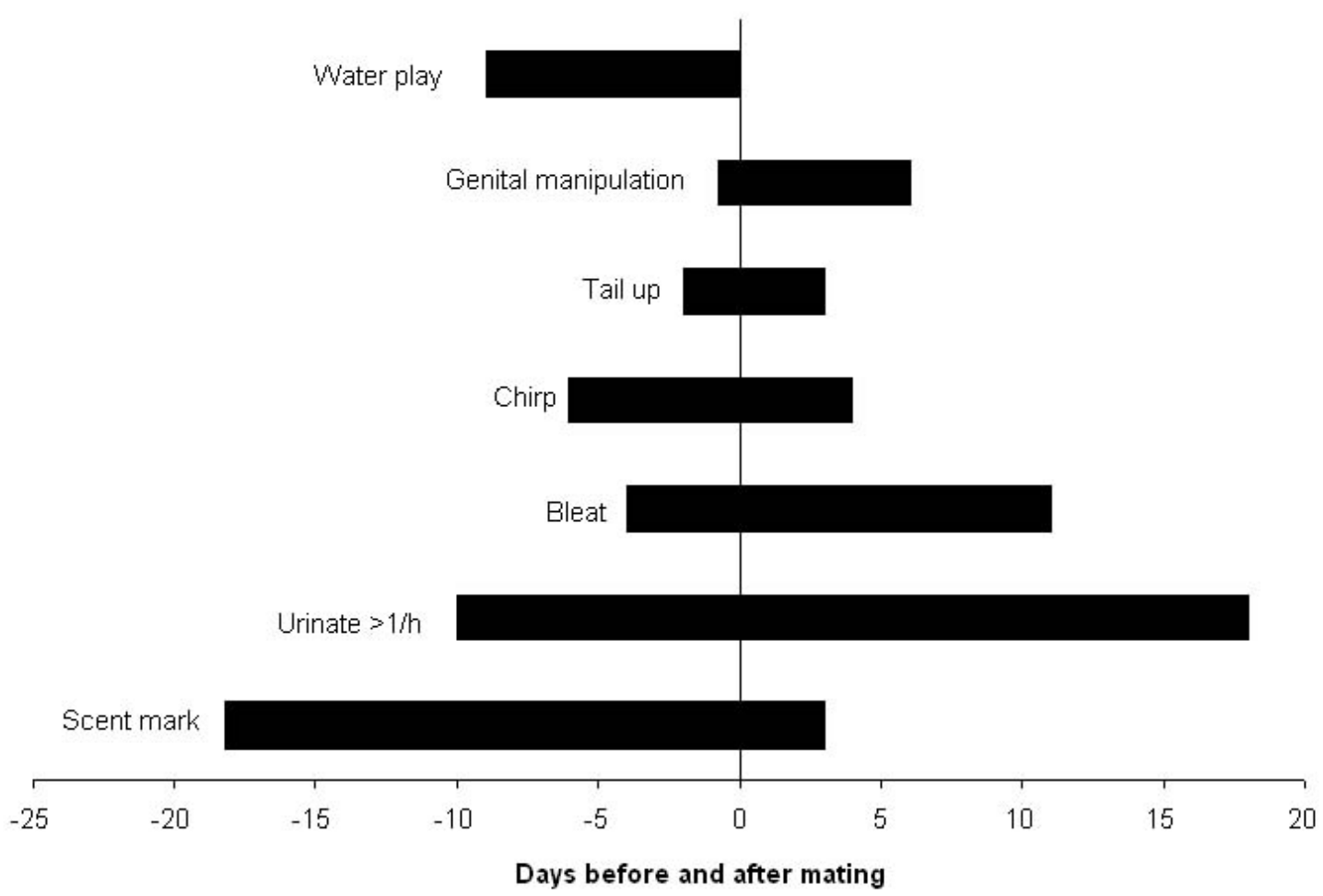

Figure 1. The onset and termination of female behaviors seen during estrus. Depicted here is the range of days in which each of these estrous behaviors were observed, with outliers excluded. Data are from 14 females and 27 estrous periods, collected over four years. Day " 0 " refers to the first day of mating, negative values to days prior to mating, and positive values to days after first mating. (from Swaisgood et al. 2003).

Mating behaviors during breeding introductions are similar to pre-mating behaviors. When males and females are introduced females will often solicit males by chirping, bleating, presenting the hindquarters, and adopting the lordosis posture with the tail up. If females are receptive to the male, males will attempt to mount the female and thrust continuously until intromission is achieved. Upon intromission, males adopt a "roll-back" position where females are pulled back into the lap of the male. Intromission is accompanied by a very distinctive, high-pitched, prolonged bleating from both the male and female panda.

Breeding managers at the CCRCGP use pre-mating behaviors to evaluate whether a pair may be suited for a mating introduction and evaluating the frequency of positive 
pre-mating behaviors has become a critical tool for determining whether a potential mate pair should be introduced for breeding. During this study approximately $38 \%$ of all breeding introductions resulted in intromission success. The percentage of intromission success for wild giant pandas is unknown making comparisons impossible.

\section{Mate Preference in Captive Breeding Programs}

Mate preference may have important consequences for conservation breeding because it can preclude breeding entirely (Swaisgood \& Schulte 2010). The reproductive performance, or lack thereof, of pairs put together to breed, especially genetically important pairs, can have significant consequences for the demographic and genetics of a captive population (Asa et al. 2011). Thus, understanding the mating system and social organization surrounding natural mating events is often crucial to the successful breeding of a species (Swaisgood \& Schulte 2010). How females and males go about selecting mates affects the success of a pairing and these selection processes can be significantly altered in the captive environment. The number of available mates, how potential mate partners signal quality, and social structures prior to mating can be drastically different in captivity compared to the wild and may have significant impacts on the choice made by the selecting individual. It is largely unknown to what extent preference mechanisms may contribute to failed mating and mate incompatibility of pairs (Swaisgood \& Schulte 2010).

Evidence is mounting that providing animals with the ability to choose their mates can greatly enhance the reproductive performance of the choosing individual, as well as affecting the viability of the resulting offspring (Drickamer et al. 2000a; Drickamer et al. 2003; Gowaty et al. 2003; Ryan \& Altmann 2001; Wedekind et al. 2001). However, this 
role has only recently been explored in zoo breeding programs (Martin \& Shepherdson 2012). In endangered Columbia Basin pygmy rabbits, we found that both familiarity with potential mates and providing the opportunity for female rabbits to mate with behaviorally preferred males increased reproductive performance (Martin \& Shepherdson 2012). These results suggested that an integrated approach to breeding assignments in the pygmy rabbit captive breeding program which combined both genetic information with behavioral measures of preference would be more successful. Subsequently, the application of this approach increased the reproductive output of the program and resulted in a surplus of animals for reintroduction into the wild (Joanne Wisnlweski pers. comm. 2014).

It seems reasonable to assume that conservation breeding programs in zoos and breeding centers have much to gain from such an integrated approach, addressing both the needs of genetic management and natural mating preferences. However, at times these goals may conflict (Swaisgood \& Schulte 2010; Wedekind et al. 2001). Allowing free mate choice (and male-male competition) may lead to unwarranted loss of genetic diversity in small populations if it results in excessive reproductive skew, with a few individuals obtaining most of the matings and contributing disproportionately to the genetic population. Still, free mate choice may also offset some of the deleterious consequences of genetic adaptation to captivity (Frankham 2008). While free mate choice may have drawbacks as well as benefits, precluding choice altogether may reduce both the quantity and quality of offspring. The challenge for the future will be to find the right balance between these two competing needs in small population management. At present, we know little about how mate preference operates in the wide variety of species 
represented in zoos, and even less about how mechanisms of mate preference could be used to manipulate breeding to increase success of genetically valuable individuals. This dissertation aims to address the question of whether mate preference, familiarity, and personality types of giant pandas affect their reproductive performance in captivity.

\section{Factors Affecting Mate Preference}

Many factors have been hypothesized to play a role in mate preference both in the wild and in captivity. I have chosen to focus my dissertation on a subset of these factors because of their prevalence and importance in current zoo research: the ability to evaluate mates (mate preference), familiarity, and personality traits.

In addition to mate preference, familiarity with mates, particularly in territorial animals, has been shown to influence female mate choice and can be altered by housing environments during breeding introductions through exposure to specific males (Cheetham et al. 2008; Wielebnowski 1999). Females can discriminate familiar from unfamiliar males and often choose their mates accordingly. Females recognize males on the basis of acoustic signals (ungulates, Reby et al. 2001; bears, Charleston et al. 2009), visual signals (fish, Zajitschek et al. 2006), and olfactory signals (rodents, Rich \& Hurst 1998; bears, Swaisgood et al. 2000). Fisher et al. (2003a) showed that female pygmy loris (Nycticebus pygmaeus) could be induced to preferentially associate with a particular

male by exposing her to his urine for several weeks. Similarly, Roberts and Gosling (2004a) manipulated the preferences of female harvest mice (Micromys minutus) by inducing males to increase olfactory signaling, thus increasing the familiarity of a particular male to the target female. In addition, during staged mate presentations, females of some bird species have been shown to increase their tendencies to affiliate as 
potential sex partners with males they have seen mating successfully with another female (Doutrelant \& McGregor 2000; Galef et al. 1998; Höglund et al. 1990). Because giant pandas have home ranges that overlap with conspecifics, familiarity may play a large role in their mate preferences. Both male and female giant pandas have been shown to differentiate between or distinguish individuals and opposite sex conspecifics based on olfactory cues left in scent marks (Swaisgood et al. 1999; Swaisgood et al. 2000), indicating that the ability to discriminate between potential mates exists. To my knowledge, familiarity of mates and how it affects mate preference has never been investigated in giant pandas.

Recently, there has been increased interest in the implications of individual differences in behavior for captive management and breeding of animals in zoos and breeding facilities (Carlstead et al. 1999a; Carlstead et al. 1999b; Gold \& Maple 1994; Mendl et al. 1992; Powell et al. 2008). For example, male black rhinoceros that were scored as being more dominant to conspecifics by their keepers were shown to have lower reproductive performance (Carlstead et al. 1999a). Similarly, Wielebnowski (1999) found that nonbreeding cheetahs were rated by their keepers as being significantly more tense or fearful than breeding cheetahs. As a complication, McDougall et al. (2006) suggests that breeding programs that ignore temperament risk leading the population towards domestication because certain personalities may be more likely to prosper in captive settings through differential reproductive performance. Therefore, the knowledge gained from personality studies can be important in optimizing captive reproduction, increasing reintroduction success and reducing the possibility of domestication (McDougall et al. 2006). In a recent study on giant pandas, Powell et al. (2008) found 
that bolder female pandas were less likely to be aggressive and were more likely to display interest in the males with which they were paired. This study also showed that giant pandas living in enclosures with only one den site were shyer, suggesting that changing enclosure features might indirectly improve reproductive performance by effecting personality. Powell et al. (2008) did not track the reproductive performance of the animals past copulation success. Thus, one of the goals for the current study is to correlate personality types with the reproductive performance of the giant panda.

Although most pandas at the CCRCGP have mated naturally, the breeding results are inconsistent. All mating attempts do not lead to successful pregnancies. As mate preference can lead to higher copulation, insemination and pregnancy rates in other species (Drickamer et al. 2000a; Martin \& Shepherdson 2012), I researched the effects of mate preference on reproductive output in pandas at the CCRCGP. To do this, we performed dichotomous choice tests on male and female giant pandas to determine mate preference and tracked various measurements of reproductive performance. The data obtained from the reproductive performance of preferred animals will be correlated with familiarity and personality of the individual bears and various breeding management techniques. Their success will be compared to the reproductive performance of nonpreferred animals. To do this, I have the following specific aims:

1. Examine mate preference and familiarity (Chapter 2) on reproductive performance of both male and female giant pandas.

2. Determine whether giant panda personality, as measured by keeper surveys and novel object enrichment tests, affects both long-term (Chapter 3) and 
individual mate pairing (Chapter 4) reproductive performance in giant pandas.

I conducted this study at the CCRCGP in Bifengxia, Sichuan, China on the giant panda. I will discuss and review the above aims in the following three chapters of this dissertation. 


\section{CHAPTER 2: DOES CHOICE MATTER? THE ROLE OF MATE PREFERENCE ON REPRODUCTIVE PERFORMANCE OF MALE AND FEMALE GIANT PANDAS}

Running head: Mate preference and reproductive performance in giant pandas

Keywords: Mate preference, mate familiarity, reproductive performance, captive breeding, giant panda, mate choice

Word Count: 9,520

Page Count: 38 


\section{Abstract}

A key component of a successful captive-breeding and re-introduction program is the ability to breed a surplus of genetically suitable animals for release into the wild. Unfortunately, many individuals often do not reproduce even when they are apparently healthy and presented with genetically appropriate mates. Mate preference can affect multiple parameters of reproductive performance, including mating success, offspring production, offspring survival, and offspring fecundity. We investigated the role of familiarity and preference on reproductive performance of male and female giant pandas (Ailuropoda melanoleuca) as measured by intromission success and litter production. We conducted these studies on giant pandas at the China Conservation and Research Center for the Giant Panda (Bifengxia, Sichuan, China) from February-May 2011, 2012, and 2013. Male and female giant pandas were housed in an alternating pattern of male, female, male, female, etc. and mate preference was determined on the basis of behavioral interactions prior to mating.

Using generalized linear mixed models, we compared reproductive performance between individuals mated with preferred and nonpreferred males from both the focal female (female preference) and the focal male (male preference) perspective. Using ANOVAs, we further investigated the effect of mutual mate preference on reproductive performance through four types of reproductive pairings; females and males that preferred each other (P-P), females that preferred the male but the male did not prefer the female (P-NP), females that did not prefer the male but the male preferred the female (NP-P), and females and males that did not prefer each other (NP-NP). Pairing giant pandas with a preferred partner increased the probability of intromission success and 
producing a cub. Of the 25 females mated to a preferred partner, $72 \%$ had successful intromission, $64 \%$ produced cubs, and $52 \%$ reared their own cubs versus $31 \%, 12 \%, 12 \%$ for females mated to their nonpreferred partner $(\mathrm{N}=16)$. Male giant panda mate preference showed similar results. Of the 24 males that were mated with their preferred females, $75 \%$ had successful intromission, $77.8 \%$ produced cubs, and $92.9 \%$ produced cubs that were maternally reared versus $31 \%, 60 \%, 66.7 \%$ for males mated with their nonpreferred females $(\mathrm{N}=16)$. There was a statistically significant difference in intromission success and cub production for P-P pairings versus NP-NP pairings. Of the twelve P-P mate dyads $83 \%$ had successful intromission, $90 \%$ produced cubs, and $88.9 \%$ had mothers rear their cubs. Of the four P-NP mate dyads 50\% had successful intromission, $50 \%$ produced cubs, and $100 \%$ had the mother rear their cub. Of the seven NP-P mate dyads $57 \%$ had successful intromission, $50 \%$ produced cubs, and $100 \%$ had the mothers rear their cubs. Of the three NP-NP mate dyads $0 \%$ had successful intromission resulting in $0 \%$ producing cubs and having mother-rear cubs.

In addition, we took into account various life and breeding history factors that may have affected reproductive performance such as birth location, rearing, previous sexual history with the opposite sex and familiarity status directly prior to breeding. Mate pairings that had successfully produced a cub in the past had increased intromission success. In addition, this familiarity measure also increased cub production for female giant pandas. Male mass was correlated with female and male reproductive performance. Females mated to heavier males had more intromissions and produced more cubs than females mated to smaller males. In captive breeding programs, mates are traditionally selected solely on the basis of genetic parameters to minimize loss of genetic diversity 
and inbreeding coefficients. Our results suggest that integrating genetic with behavioral measures of preference and breeding history factors would increase the reproductive output of the giant panda captive-breeding program. 


\section{Introduction}

Typically, captive breeding of endangered species has focused on genetic characteristics rather than behavioral characteristics such as mate preference and competition (Snyder et al. 1996b). Yet despite years of research and countless dollars into captive breeding, efforts to create self-sustaining populations of endangered species through captive breeding have been less than optimally successful. Lees and Wilcken (2009a) analyzed 87 mammal populations in zoos and found that $52 \%$ were not breeding to replacement and that $67 \%$ fell below the threshold of 200 individuals deemed necessary for genetically healthy and sustainable populations proposed by Baker (2007). In captive-breeding programs, animals are typically paired for mating to minimize inbreeding and maintain founder representation (i.e., minimize loss of genetic diversity). Such goals usually preclude mate preference (Carlstead \& Shepherdson 1994). Many zoo and breeding facility programs give animals a single option for a mate and the success or failure of reproduction hangs on the outcome of that one pairing. All too often, individual animals will forgo mating in captivity and entire lineages end with individuals that fail to mate, along with the genetic diversity scientists are striving to save. Recent studies have shown that giving an animal a choice between two or more opposite sex conspecifics can significantly increase the reproductive performance of the choosing individual (Drickamer et al. 2000a; Drickamer et al. 2003; Gowaty et al. 2003; Martin \& Shepherdson 2012). Incorporating mate preference into captive breeding programs may be an important tool to increase the success of these programs.

Mate preference has been recognized as an important factor in reproduction and evolution since Darwin (1871) posed it as a mechanism of sexual selection. Subsequent 
studies focusing on female mate preference resulted in theories such as Fisherian runaway traits (Fisher 1915), sensory bias (Boughman 2002), direct phenotypic benefits (Price et al. 1993), indicator traits (Hamilton \& Zuk 1982), and genetic compatibility (Eizaguirre et al. 2009). Recent work has started to link fitness benefits with both male and female mate preference. Drickamer et al. (2000a) showed that both male and female house mice (Mus domesticus) mated to their preferred partner showed increased fitness measurements as indicated by increased litters, higher kit survivorship than animals mated to nonpreferred partners. Viewing mate preference from a single sexes perspective can be misleading as the act of mating and intromission requires both sexes to participate. In mammals, stringent tests of mutual mate preference are rare, even though mutual mate preference may be common place (Johnstone et al. 1996). Drickamer et al. (2003) found that mutual mate preference (preferred-preferred pairings) in house mice increased the number of pups weaned, average pup mass at birth and weaning, the proportion of females in the litter, and offspring performance in dominance tests and predator avoidance tests versus mate pairings where partners were not preferred (nonpreferrednonpreferred pairings). Cumulatively, these studies suggest that there are clear fitness benefits to both male and female as well as mutual mate preference. It is easy to see how applying similar methodology to captive breeding settings, where mate preference is traditionally underemphasized, could potentially increase reproductive performance in endangered species.

Mate incompatibility impedes captive breeding programs (Gosling \& Sutherland 2000; Wielebnowski \& Brown 1998) by reducing reductive rates and excluding potential genetic contributions to the population, compromising the goals of maintaining genetic 
diversity for assurance and reintroduction source populations. It is therefore surprising that mate preferences have not figured prominently in captive breeding programs, as failure to address mate preferences can lead to reduced offspring production and viability. However, recent research has made inroads into this arena, demonstrating the potential value of systematic studies investigating how mate preferences influence these important goals of captive breeding programs. For example, in the endangered Columbia Basin pygmy rabbits, we found that both familiarity and mating with a preferred partner increased intromission success, litter production, and kit survivorship (Martin \& Shepherdson 2012). These results suggest that an integrated approach to breeding assignments in the pygmy rabbit captive breeding program, one that combines both genetic information and behavioral measures of preference, has the potential to increase the reproductive output of the captive breeding program. Taken together, the studies reviewed above spanning multiple species show that mate preference can affect copulation success, offspring production, and offspring survivorship. The species studied to date in the laboratory and in captivity have been small, short-lived species (relatively $r$-selected species) not the large, long-lived species (relatively $k$-selected species) that are more typically found in endangered species captive breeding programs.

For the recovery of the iconic endangered species, the giant panda (Ailuropoda melanoleuca), captive breeding is an important tool for augmenting declining wild populations and reestablishing extirpated populations (Gilpin \& Soule 1986). Like other endangered species, giant pandas have been notoriously reluctant to breed in captivity (Swaisgood et al. 2003a; Swaisgood et al. 2006). In 1996, Chinese researchers estimated that only $39 \%$ of captive female pandas produced offspring, $80 \%$ failed to display normal 
behavioral estrus, and only six living males in captivity had ever mated naturally (Lindburg et al. 1998). For unknown reasons (i.e. individuals seemed physiologically capable of reproduction), many giant pandas in captivity do not reproduce (Ellis et al. 2006). There is good reason to believe that giant pandas, like almost all carefully studied species, have mate preferences. Mate preference in giant pandas is often cited as a limiting factor in captive reproduction, and Zhang et al. (2004b) reported that they found one female giant panda that consistently displayed low levels of estrous behavior in the presence of some males but quickly changed to more obvious estrous behaviors when exposed to other males, suggesting strongly that female preference exists in giant pandas. Earlier research demonstrated that behavioral compatibility can be related to the success or failure of attempted pairings (Swaisgood et al. 2006; Swaisgood et al. 2003b; Zhang et al. 2004b). Swaisgood et al. (2003b) report that there is a variation in male behavior toward estrous females, with some males showing little interest in the female and not copulating, but with most males displaying consistent signs of interest in the female, approaching her frequently, sniffing at her through the cage bars, and occasionally bleating (an affiliative vocalization). These findings imply that males may also actively prefer certain females over others. A recent study at the Chengdu giant panda breeding center in Chengdu, China, showed that for successful copulation and subsequent cub production, both the male and female panda had to show a high level of courtship behavior during mating introductions (Peng et al. 2009). However, to our knowledge no study has critically investigated whether mate preference, regardless of frequency of courtship behaviors, affects reproductive performance of a mating pair.

The China Conservation and Research Center for Giant Pandas in Wolong 
(CCRCGP, now relocated to Bifengxia following the earthquake that destroyed the original facility) has long been China's most successful breeding facility. The CCRCGP offers an ideal test of the effects of mate preferences on reproductive performance in a large, long-lived species because it has a large adult breeding population in excess of forty individuals, its giant pandas often fail to mate even though physiologically capable, and the captive breeding program allows for experimental designs that can test mate preference in highly controlled breeding situations. The CCRCGP uses breeding methods that allow researchers to opportunistically collect data similar to dichotomous choice tests used in laboratory settings. During the breeding season the CCRCGP houses animals in an alternating male, female, male, female, etc. pattern such that female and male preference can be measured prior to mating attempts. Neighboring conspecifics are recommended for breeding by the Species Survival Plan ${ }^{3}$ and animals are observed for signs of behavioral incompatibility that would indicate animals should not be introduced for mating. Though the CCRCGP has utilized this system for years, no studies have been conducted that investigate correlations of mate preference with reproductive performance.

In this study, we took advantage of the current breeding arrangement at the CCRCGP and conducted dichotomous mate choice tests to investigate whether the reproductive performance of male and female giant pandas mated with preferred partners differed from individuals mated with nonpreferred partners on the basis of intromission success and litter production. We expected that individual introduced to their preferred

\footnotetext{
${ }^{3}$ A cooperatively managed breeding plan that identifies population management goals and recommendations to ensure the sustainability of a healthy, genetically diverse, and demographically varied breeding population
} 
mate would have higher reproductive performance than individuals introduced to nonpreferred mates.

\section{Methods}

\section{Study Site and Species}

Giant panda research was conducted during the breeding season (1 February-1 May) of 2011, 2012, and 2013. Due to intraspecific aggression, pandas were separated from each other except when males were introduced to female enclosures for breeding. Giant pandas were housed at the CCRCGP in concrete walled, open-air enclosures ( $8 \mathrm{~m} \mathrm{x}$ $25 \mathrm{~m}$ ) that contained various forms of environmental enrichment (e.g. climbing platforms, water features, trees, etc.). All enclosures included an indoor enclosure area $(3 \mathrm{~m} \times 8 \mathrm{~m})$ to which animals had free access during all hours except during cleaning. All enclosures were exposed to natural light only.

Anywhere from 1-2 weeks prior to breeding (as determined by manager observations of female behavior and progesterone profiles) males and females were moved to pens that consisted of an outdoor area $(20 \mathrm{~m} \times 25 \mathrm{~m})$ and indoor area $(3 \mathrm{~m} \times 5 \mathrm{~m})$. Animals were free to move between the outdoor and indoor areas. The outdoor enclosure walls were $4.5 \mathrm{~m}$ tall concrete walls that had walking platforms on top of the wall for viewing panda breeding behaviors more readily. All enclosures had three barred "howdy" windows and a circular barred gate located on the long sides of the enclosure (8 potential interaction windows, 4 per side). Thus, pandas were able to interact through cage bars with neighboring individuals in adjoining enclosures, but opportunities for physical contact were limited (See Appendix E). 
Enclosures were arranged in a large U-shape so pandas could be moved freely between pens for mating pairings. In this configuration, pandas shared walls with two other animals except for the end enclosures, which only had one neighbor. Pandas were fed five large bamboo culms ( $\sim 3$ meters long and $10 \mathrm{~cm}$ diameter) in the morning from 9:30-11:00h directly after cages were cleaned and in the afternoon at 15:00-16:00h. Panda diets were also supplemented with bread, high-fiber biscuits, carrots, and apples. Animal care and use guidelines of the American Society of Mammalogists (Animal Care and Use Committee 1998; Assurance \#: A3675-01) were followed by all facility operators.

\section{Mating Procedure}

Here we define mate pairings as the introduction of a specific male to a specific female for the purpose of breeding. In the wild and captivity, the breeding season for the panda is from February to May. We began assessing mate pairing of adult giant pandas in mid-February and continued until the end of April in 2011, 2012, and 2013 (we had two females enter estrus in May over the course of the study). The assignment of individuals to mate pairs was based on a captive breeding genetic management plan determined by breeding managers and designed to maximize genetic health of the captive population. Mating was always attempted first with the priority male on the genetic management plan even if animals appear indifferent or slightly aggressive toward the opposite sex conspecific. Potential mates were housed next to each other to determine if the pair displayed too much aggression to attempt a mate introduction. If the male and/or female displayed excessive aggression (continuous growling and swiping through the cage bars), breeding managers did not attempt a mate introduction. However, affiliative behavioral 
displays by the potential mating pair were not necessary for an introduction and pairs were often introduced and sometimes have successful copulation even when both the male and female pandas appeared ambivalent toward their potential mate partner. To determine female estrus status, urine samples of female giant pandas were collected via syringe from the cages of female giant pandas during the breeding season, $\sim 3$ days a week, until baseline levels rose and then samples were collected daily through peak estrus and hormone decline. Samples were used to determine the date of estrus. Urine samples were immediately processed by the CCRCGP for estrogens and progestins by enzymeimmunoassay previously validated on urine (McGeehan et al. 2002).

Males were introduced to female pens for mating between 9:00 and 11:00h. Mating sessions lasted on average 15.5 minutes but ranged from 3-75 minutes. If either animal's behavior was aggressive, animal care staff removed the male immediately to prevent injury or death. After a mating session, males were moved back to their enclosures and subsequently placed with a different female until all females had been mated to their recommended males. This method resulted in females being introduced to 1-4 males and having on average 4 (but as many as 9) mating opportunities each breeding season. As a fail-safe, female pandas are often artificially inseminated in addition to natural breeding. If paternity was in question, the CCRCGP established the father using DNA obtained from hair samples and amplified utilizing the polymerase chain reaction to analyze microsatellite loci after the methods of Zhang et al. (1994). All cubs used in this study had confirmed paternity.

Mating behaviors were defined as in Swaisgood and Owen (2007). All occurrences of these behaviors were recorded along with the identity of the panda performing the 
behavior. In addition, we recorded latency to contact, latency to mounting, latency to copulation, and total copulation time. Recording started as soon as females and males were given access to each other in the outdoor mating enclosures and ended once keepers attempted to separate the animals. If copulation occurred recordings were ended as soon as the male and female broke contact as female pandas become very aggressive at the termination of copulation. Behaviors were scored per mating dyad, which was defined as one male and one female giant panda paired for the purpose of mating.

We monitored several measures of reproductive performance; whether a mating attempt failed or succeeded (i.e. copulation occurred with intromission), whether cubs were produced, and whether cubs were hand raised or mother raised. We also consulted studbooks and veterinary records to determine if animals had previous natural mating experience, had successfully produced cubs in the past, age at first mating, and the number of previous years animals had natural mating experiences. During the breeding season we collected data for each focal animal on the total number of natural mate attempts conducted, the total number of mating attempts that were successful, the number of days over which mating was attempted, and the sequential number of mates the focal animal had encountered prior to the specific mate dyad being recorded.

\section{Mate Familiarity}

Studbooks and veterinary records were consulted to determine if mate dyads had previously produced litters successfully. We collected data on several factors that may indicate familiarity between mate dyads that were obtained from studbook and veterinary records or from accurate recording of enclosure moves throughout the year. Factors included: previous litter production with the potential mate (binary response variable: yes 
$=1$, no $=0$ ), familiarity status during the year prior to breeding (in days), familiarity status during the month prior to breeding (in days), familiarity status directly prior to breeding (number of contiguous days animals were neighboring each other). Familiarity status directly prior to breeding was highly variable ranging from 30 minutes to ten days. Locations for all giant pandas were tracked from January $1^{\text {st }}-$ December $31^{\text {st }}$ in 2010 , 2011, 2012, and 2013. The month of January was inconsistently collected across years so we did not include this month in our calculations. Locations were recorded on a weekly basis during the nonbreeding season (June-December) as movement of bears to different enclosures is rare, and on a daily basis during breeding season (February-May) when movement of bears to breeding enclosures is common.

\section{Mate Preference Procedure}

As described above, female and male giant pandas included in mate preference trials were housed between conspecifics of the opposite sex and at least one of these conspecifics was designated as a potential mate for that season. Females and males were observed and mate preference behavior was scored 1-3 days before an opposite sex conspecific was introduced to the focal animal for mating introductions. All occurrences of the focal animal's behaviors as defined below were scored for 30 minutes between 7:30-11:00. This is the period when pandas were most active in these facilities and also coincided with breeding manager's evaluation time.

Pre-mating behaviors were defined from the San Diego Zoo Giant Panda Behavioral ethogram (Swaisgood \& Owen 2007) based on their importance as indicators of impending estrus in females and sexual arousal in males: scent-marking, urination, water play, rolling, feeding activity, activity level, interest and interaction with opposite 
sex conspecifics, chirping, bleating, masturbation, tail-up (female only), lordosis (female only), backward walking (female only), penile erection (male only) and foot scraping (male only). Also included were behaviors that may indicate negative interactions such as aggression through attempted physical attacks, lack of interest in opposite sex conspecifics, moaning, barking, growling, roaring and avoidance.

We adapted methods from Drickamer et al. (2000a) to determine preference for neighboring potential mates. Animals were defined as preferred by the focal panda if the focal animal directed at least $60 \%$ of their total behaviors during the 30 -minute observational period toward one particular potential mate. If the focal panda did not demonstrate such a preference they were excluded from the study $(\mathrm{N}=5)$. The observations were conducted with a single-blind trial protocol, whereby observers were naive to the identity of the conspecific that would be paired with the focal panda. Focal pandas were assigned to be mated with either the preferred or non-preferred mate on the basis of which male was genetically recommended by the species survival plan.

Data measurements were subdivided into three sets: 1) female mate preference where female pandas were the focal animal $(\mathrm{N}=41), 2)$ male mate preference where male pandas were the focal animal $(\mathrm{N}=40)$, and 3) mutual mate preference obtained from data that were used in $1 \& 2$. The sample size was lower for this set because we did not always have focal behavioral scores for both individuals in a mate dyad ( $\mathrm{N}=26$ mate dyads). For the third data set, 'mutual mate preference', we divided mating dyads into four possible mating categories after Drickamer et al. (2003): 1) both giant pandas preferred each other ('P-P' indicated that the male was preferred by the female and the female was preferred by the male, 2) the female preferred the male but the male did not 
prefer the female (P-NP), 3) the female did not prefer the male but the male did prefer the female (NP-P), and 4) neither the female nor the male preferred each other (NP-NP).

Here and throughout the text and tables we designated the female preference first and the male preference second.

\section{Data Analyses}

Prior to analysis variables were examined for normality, linearity, and homoscedasticity. We used both Bayesian modeling and step-wise reduction of generalized linear mixed models (GLMMs). Bayesian modeling has recently seen increased use in the epidemiological literature and is considered a strong statistical tool because it simultaneously tests both uncertainties related to models and explanatory parameter values. In contrast, most analyses based on maximum likelihood (or least squares) estimation, such as GLMMs, involve fixing the values of parameters that may have considerable uncertainty and an impact on the final outcome of the analysis. In addition, Bayesian methods have lower requirements for sample sizes and are, therefore, desirable when dealing with endangered species studies. Thus, for data where multiple explanatory factors could be affecting a response variable, Bayesian methods are usually considered superior methods to GLMMs. However, we ran GLMMs as well to more easily compare our previous research results and because conservation biologists have traditionally used GLMMs.

We analyzed 3 measures of reproductive performance using Bayesian Model Averaging (Lenkoski et al. 2014) in R Studio (Version 0.98.981, R Studio Inc 20092013; R Version 3.0.2). The first response variable was whether a mating attempt resulted in copulation (binary response variable: yes $=1$, no $=0$; generalized linear mixed model 
with logit link function). The second response variable was whether cubs were produced (binary response variable: yes $=1$, no $=0$; model with logit link function). The third response variable was whether or not a female raised her cub or humans intervened and hand raised the cub due to maternal abandonment or incompetence (binary response variable: hand reared $=1$, mother reared $=0$; model with logit link function). The potential explanatory variables/factors that could affect the success of a mating attempt were: previous litter production with the potential mate (binary response variable: yes = 1 , no $=0$ ), familiarity status during the year prior to mating sessions (in days), familiarity status during the month prior to mating sessions (in days), familiarity status directly prior to mating sessions (in contiguous days animals were neighboring each other), mate preference status (binary response variable: preferred $=1$, nonpreferred $=0$ ), year of mating session $(2011,2012,2013)$, focal animal birth location (i.e. provenance; binary response variable: captive $=1$, wild $=0$ ), focal animal rearing (binary response variable: mother $=1$, hand $=0$ ), female age (years), female mass (kilograms), female length (centimeters), female height (centimeters), male age (years), male mass (kilograms), male length (centimeters), male height (centimeters), total number of natural mating sessions conducted that year for the focal panda, the sequential number of mates the focal animal had encountered prior to the specific mate dyad, number of days to or from peak estrus mating when the mating introduction was attempted, the focal panda's long-term previous natural mating experience (binary response variable: yes $=1$, no $=0$ ), if the focal animal had successfully produced cubs long-term (binary response variable: yes = 1, no $=0$ ), and total number of prior breeding years. Some variables showed a high degree of inter-correlation. In these cases, the variable with the most relevance to the 
hypothesis and most normal distribution was selected for analysis. For example, male mass was significantly and highly correlated with male length $(r=-0.59, p<0.001)$ and height $(r=-0.64, p<0.001)$. Thus, male mass was the only factor used to describe male size. Likewise, female mass was significantly correlated with female length $(r=0.22$, $p=0.05)$ and height $(r=-0.29, p=0.03)$, and therefore, female mass was the only factor used to describe female size. All explanatory variables were then fitted in all possible combinations to create a list of explanatory models. We used the Bayesian information criterion $(B I C)$ to rank the explanatory models.

Regression analyses generally assume that all observations are independent; however, this is not the case for pairwise data where the same individual may be involved in multiple mating attempts. To account for this dyadic non-independence, we took giant panda ID into account by incorporating it as a random effect in our analyses (Browne et al. 2001; Clarke et al. 2002). This allowed us to separate the effect of ID as a confounding variable (i.e. if an individual was a really "good breeder" versus a "bad breeder").

From the Bayesian models we selected the most explanatory factors across the first five most highly ranked models predicting reproductive performance measurements. Because GLMMs have traditionally been used in the conservation and mate preference literature we analyzed the most explanatory factors obtained from our Bayesian models in GLMMs. All GLMMs had random effects of female and male identity included in the minimal model. We ran GLMM through a step-wise exclusion method in which the least significant predictor variable $(p \geq 0.05)$ was sequentially removed from the model until the final significant model was revealed (McCullagh \& Nelder 1989). Models were 
compared using penalized log likelihood scores (Bayesian Information Criterion (BIC); Crawley 2005). Minimal models were checked by adding all terms back in, one by one, and examining model QIC scores for possible improvement in model fit.

For our mutual mate preference data analyses we tested mating dyad reproductive performance measurements using one-way ANOVAs with a single factor with the four mating types as treatment (P-P, P-NP, NP-P, NP-NP). The ANOVAs that were significant or trending toward significance $(p \leq 0.07)$ were followed by Tukey HD post hoc tests to examine mean differences between treatments.

All analyses were performed in either R Studio (Version 0.98.981; R Studio Inc. 2009-2013; R Version 3.0.2) or SPSS 19.0 for Mac OS X.

\section{Results}

\section{Female Reproductive Performance}

Our Bayesian models (Appendix A; Table A.1 and Table A.2) suggested that previous litter production, mate familiarity status directly prior to mating sessions (contiguous days animals were neighboring each other), mate preference status, focal animal rearing, male age, and male mass were the explanatory factors that most explained intromission success and cub production. To be conservative and because past studies on giant pandas had found birth location, female age, and female mass to be important factors predicting reproductive performance, we included these factors in our GLMM model in addition to the factors suggested by the Bayesian models. Complete GLMM models and removed explanatory factors for intromission success and cub production can be found in Supplementary Material (Appendix A, Table A.3 and Table A.4 
respectively).

The explanatory variable of mate preference was the most correlated variable with reproductive performance measurements on the top Bayesian model (Appendix A Table A.1 and Table A.2). However, it was the second most explanatory factor in our GLMMs. Measures of reproductive performance varied between females mated with preferred and nonpreferred males (Table 2.1). Significantly more females mated to their preferred male had successful intromission (Figure 2.1; $\beta=-2.23$, Wald Chi-Square $_{1,41}=7.22, p=$ 0.007; Appendix A, Table A.3), and produced more cubs (Figure 2.1; $\beta=4.91$, Wald Chi-Square $_{1,41}=11.35, p=0.001$; Appendix A, Table 4 in supplementary material) than females mated to nonpreferred males, but did not raise their cubs more often (Figure 2.1; $\mathrm{X}^{2}=0.45, p=0.50$ ). Mate preference had a large effect on reproductive measurements. Twenty-five females were mated with preferred males; 18 (72\%) had successful intromission, $16(64 \%)$ produced cubs, and $13(52 \%)$ reared their own cubs (Figure 2.1, Table 2.1). Of the sixteen females mated with nonpreferred males; 5 (31\%) had successful intromissions, $2(12 \%)$ produced cubs, and $2(12 \%)$ reared their own cubs (Figure 2.1, Table 2.1).

Most of our familiarity measures had no effect on whether a female copulated or produced cubs with a particular male. Familiarity status during the year prior to the mating session and familiarity status during the month prior to the mating session did not load on any of the top five Bayesian models. The number of continuous days of familiarity status directly prior to the mating session loaded on the fifth recommended Bayesian model but was not significantly associated with either intromission $(\beta=-0.24$, Wald Chi-Square $\left._{1,41}=3.73, p=0.208\right)$ or cub production $\left(\beta=0.24\right.$, Wald Chi-Square $_{1,41}$ 
$=1.10, p=0.294)$ in the GLMM.

The explanatory variable of mate pairs previously producing a litter significantly loaded on the top Bayesian model after mate preference (Appendix A, Table A.1 and Table A.2). Moreover, this factor highly predicted both female intromission success and cub production (i.e. all animals that had previous litters together had successful intromission and produced cubs; Figure 2.2), thus, we were unable to run full model GLMMs on this factor. However, a basic model GLMM could be run with previous litter production as an explanatory variable and giant panda IDs as random effects but excluding all other explanatory variables. This model revealed that mate pairs composed of males and females that had previously produced a litter were associated with increased intromission success (Figure 2.2; $\beta=23.64$, Wald Chi-Square $_{1,41}=4399.63, p<0.001$ ), increased cub production (Figure 2.2; $\beta=-2.30$, Wald Chi-Square $_{1,41}=5.89, p<0.015$ ), but not with increased maternal rearing (Figure 2.2; $\mathrm{X}^{2}=0.81, p=0.37$ ). Measures of reproductive performance varied between females mated to males that had previously sired one of their cubs and females mated to males that had not sired their cubs (Table 2.2, Figure 2.2). Of the ten females that were mated to males that had sired one of their previous cubs; $10(100 \%)$ had successful intromission, $9(90 \%)$ produced cubs, and 8 $(80 \%)$ reared their own cub. Of the 34 females mated to males that had not sired one of their previous cubs; $16(47.1 \%)$ had successful intromission, 11 (68.8\%) produced cubs, and $8(72.7 \%)$ reared their own cub.

The third most explanatory variable on our Bayesian model with relatively low correlation scores was male mass (Appendix A, Table A.1 and Table A.2). This variably consistently loaded on all top five models. This factor had the most explanatory power in 
our GLMMs. Females mated with larger males were more likely to have successful intromission (Figure 2.3; $\beta=0.10$, Wald Chi-Square $_{1,41}=11.2, p<0.001$; Appendix A, Table A.3 in supplementary material), were more likely to produce cubs (Figure $3 ; \beta=-$ 2.30, Wald Chi-Square $_{1,41}=5.89, p<0.001$; Appendix A, Table A.4 in supplementary material), but were not more likely to rear cubs (Figure 2.3; $\mathrm{U}=23, p=0.29$ ). Male age was also the third most explanatory factor for cub production with older males producing more cubs $\left(\beta=0.10\right.$, Wald $_{\text {Chi-Square }}, 41=11.2, p<0.001$; Appendix A, Table A.4 in supplementary material). However, male age and male mass were highly correlated in our data $(r=0.27, p=0.015)$.

The last and final explanatory factor that explained cub production but not intromission success in our GLMMs was whether the female panda was hand reared or mother reared. Females who were mother reared as infants produced cubs more often than females that were hand reared $\left(\beta=-3.74\right.$, Wald $_{\text {Chi-Square }}, 41=5.48, p=0.019$; Appendix A, Table A.4 in supplementary material).

No other variables were significantly correlated with female intromission success, cub production, or maternal rearing (all $p$ values $>0.05$ ).

\section{Male Reproductive Performance}

Fitness measurements with data obtained from males as focal animals showed similar patterns to female fitness as these two data sets were highly interrelated (i.e. reproductive performance measurements cannot be fully separated out into 'male' and 'female'). Our Bayesian models suggested that mate preference status, male age, birth location, and male ID might be factors that explained intromission success and cub production (Appendix B, Table B.1 and Table 2.2 respectively). To be conservative, we 
decided to include previous litter production with the potential mate, familiarity status directly prior to mating sessions (in continuous days animals were neighboring each other), female age, and female mass in our GLMM models. The explanatory factor 'focal animal rearing' was removed from our final GLMM model because all males in our data set were mother reared which resulted in no measurable variation in this factor. Complete GLMM models and removed explanatory factors for intromission success and cub production can be found in our Supplementary Material (Appendix B, Table B.3 and Table 2.4 respectively).

Mate preference was the most explanatory factor in our Bayesian models (Appendix B, Table B.1 and Table B.2) and the second most explanatory factor in our GLMMs for both intromission success and cub production. Measures of reproductive fitness varied between males mated with preferred and nonpreferred females (Table 2.2). Males mated to their preferred female had successful intromission (Figure $4 ; \beta=1.44$, Wald Chi-Square $_{1,40}=2.70, p=0.100$; Appendix B, Table B.3 in supplementary material) and produced more cubs (Figure 2.4; $\beta=1.64$, Wald Chi-Square ${ }_{1,40}=2.32, p=$ 0.128; Appendix B, Table B.4 in supplementary material) than males mated to nonpreferred females. Because only two explanatory factors, mate preference and if the mate dyad had previously produced cubs, were loading on the GLMM with the highest $B I C$ and male mate preference was highly correlated with the pair previously producing a litter $(r=-0.23, p=0.05)$ we stepped out these two factors independently from our final model. The final GLMM with 'mate pair previously producing a litter' removed showed that mate preference produced a better (i.e. lower) BIC score with a significant positive correlation with intromission $\left(\beta=1.89\right.$, Wald Chi-Square $_{1,40}=5.72, p=0.02$, BIC $=$ 
51.81) and cub production $\left(\beta=1.98\right.$, Wald $\left._{\text {Chi-Square }}, 40=5.64, p=0.02, B I C=51.35\right)$. Similar to female preference, male mate preference had a large effect on reproductive measurements. Twenty-four males were mated with preferred females; 18 (75\%) had successful intromission, $14(77.8 \%)$ produced cubs, and $13(92.9 \%)$ produced cubs that were maternally reared (Figure 2.4, Table 2.2). Of the sixteen males mated with nonpreferred females; 5 (31\%) had successful intromissions, $3(60 \%)$ produced cubs, and $2(66.7 \%)$ produced cubs that were maternally reared (Figure 2.4, Table 2.2).

Most of our familiarity measures did not predict whether a male had successful intromission or produced cubs with a given female. Familiarity status during the year prior to the mating session and familiarity status during the month prior to the mating session did not load on any of the top five Bayesian models. The number of continuous days of familiarity status directly prior to the mating session did not load on our Bayesian models and was not significantly associated with either intromission success $(\beta=-0.04$, Wald Chi-Square $\left._{1,40}=0.14, p=0.712\right)$ or cub production $\left(\beta=0.01\right.$, Wald Chi-Square $_{1,40}$ $=0.002, p=0.964)$ in the GLMMs.

The explanatory variable of mate pairs previously producing a litter did not significantly load on the top Bayesian models (Appendix B, Table B.1 and Table B.2). However, GLMMs revealed that mate pairs composed of males and females that had previously produced a litter were associated with increased intromission success (Figure 2.4a; $\beta=2.01$, Wald $_{\text {Chi-Square }}, 40=3.32, p=0.069$ ) but not increased cub production (Figure 2.4b; $\beta=-0.17$, Wald Chi-Square ${ }_{1,40}=0.03, p=0.867$ ). As before we reduced the final model to one explanatory variable because 'mate preference' and 'pair previously produced a cub' were correlated. The final GLMM with 'mate preference' 
removed showed that if a pair had previously produced a cub showed a worse fit (i.e. higher) $B I C$ score but had a significant positive correlation with intromission $(\beta=2.58$, Wald Chi-Square $_{1,40}=5.12, p=0.02$, BIC $=61.88$ ). Measures of reproductive fitness varied between males mated to females that had previously had cubs (Table 2.2, Figure 2.5). Of the eleven males that were mated to females that had their cubs previously, 10 $(90 \%)$ had successful intromission, $8(80 \%)$ produced cubs, and $8(100 \%)$ had cubs that were maternally reared. Of the 37 males mated to females that did not have their cubs previously; 16 (43\%) had successful intromission, 11 (68.8\%) produced cubs, and 8 (72.7\%) had cubs that were maternally reared.

Focal animal birth location loaded on the third and fourth Bayesian model with low correlations scores (Appendix B, Table B.1 \& Table B.2). For our cub production GLMM the explanatory factor 'focal animal birth location' had to be removed from our model because all wild-born males $(\mathrm{N}=5)$ produced cubs whereas only one captive born male $(\mathrm{N}=4)$ produced cubs. We ran a basic model GLMM with birth location as an explanatory variable and giant panda IDs as random effects but excluding all other explanatory variables. This model revealed that males from the wild were more likely to produce a cub (Figure 2.5b; $\beta=23.64$, Wald Chi-Square $_{1,40}=4399.63, p<0.001$ ) but were no more likely to have successful intromissions (Figure 2.5a; $\beta=-0.88$, Wald ChiSquare $\left._{1,40}=0.16, p=0.687\right)$.

The most explanatory factor in our cub production GLMM was male mass (though this did not load on any of our top Bayesian models). Larger males were more likely to produce cubs (Figure 2.6a; $\beta=-0.20$, Wald $_{\text {Chi-Square }}{ }_{1,40}=5.90, p=0.015$; Appendix B, Table 2 in supplementary material) but were not more likely to have intromission 
${\text { (Figure 2.6b; } \beta=-0.10, \text { Wald }_{\text {Chi-Square }}, 40}=1.74, p=0.187$; Appendix B, Table B.1). When we removed 'mate preference' leaving only male mass in the model, the resulting model showed a worse (i.e. higher) $B I C$ score but a significant correlation with cub production $\left(\beta=-0.11\right.$, Wald $_{\text {Chi-Square }}$ S $\left._{10}=5.64, p=0.02, B I C=54.11\right)$ It is likely that this correlation is a 'hold-over' from intromission success seen in female mate preference.

No other variables were significantly correlated with male intromission success, cub production, or maternal rearing (all $p$ values $>0.05$ ).

\section{Mutual Mate Preference and Fitness}

Twelve mate dyads were composed of females and males that were mated to their preferred partner; $10(83 \%)$ had successful intromission, $9(90 \%)$ produced cubs, and 8 $(88.9 \%)$ had mothers rear their cubs. Four mate dyads were composed of females that preferred their mate and males that did not prefer their mate; $2(50 \%)$ had successful intromission, $1(50 \%)$ produced cubs, and $1(100 \%)$ had the mother rear their cub.

Seven mate dyads were composed of females that did not prefer their mate and males that did prefer their mate; 4 (57\%) had successful intromission, 2 (50\%) produced cubs, and 2 $(100 \%)$ had the mothers rear their cubs. Three mate dyads were composed of females that did not prefer their mate and males that did not prefer their mate; $0(0 \%)$ had successful intromission resulting in $0(0 \%)$ producing cubs (Figure 6, Table 3 ).

Table 2.3 presents tests for differences in components of reproductive performance among the four mating types; P-P, P-NP, NP-P, NP-NP. Mutual mate preference correlated highly with reproductive measurements. ANOVAs revealed a significant difference across the four mating types in both intromission success and cub production 
but not in whether females reared their own cubs. Tukey HD post hoc analyses indicated that P-P and NP-P pairings had significantly more successful intromissions than NP-NP mate pairings. Thus, pairings where males preferred females but females did not prefer males revealed slightly higher reproductive performance (statistically the same as P-P) than the opposite scenario where females preferred males but males did not prefer females (statistically the same as NP-NP pairings). Further, Tukey HD post hoc analyses also indicated that P-P pairings had more cubs than both NP-NP and NP-P pairings. There was no significant difference in the age of females and males or masss of females for the four mating types (Table 2.3). There was a marginally significant difference in male mass between the four mating types (Table 2.3). Tukey HD post hoc analyses indicated that P-P pairings involved significantly heavier males than both NP-NP and PNP pairings (Table 2.3).

\section{Discussion}

\section{Mate preference and variation in reproductive performance measurements}

This is the first study to test female and male giant panda preference using dichotomous choice tests and associate it with reproductive performance. Female and male mate preference yielded important reproductive benefits for giant pandas; mate preference correlated positively with a pair's subsequent reproductive performance in intromission success and cub production. Therefore, both sexes showed increased reproductive performance measurements when mated with partners they preferred versus partners they did not prefer. Mate preference and previous cub production were consistently ranked as the two highest explanatory variables in our Bayesian models and 
GLMMs. Mate preference was the highest predictor in our Bayesian models and second highest explanatory factor in our GLMMs. These findings represent the second example we have found that mate preference influences reproductive performance in an endangered species (Martin \& Shepherdson 2012). Unlike pygmy rabbits (Howard et al. 1998; Martin \& Shepherdson 2012) and house mice (Drickamer et al. 2000a; Drickamer et al. 2003; Gowaty et al. 2003), we did not find increased offspring fitness as measured by hand or mother-rearing in giant pandas. We used 'hand reared' versus 'mother reared' as a proxy for offspring survival (if cubs had to be removed from the mother and hand reared we assumed they would have perished) but because giant panda cubs are extremely valuable the staff will hand raise the babies if it appears that a mother will not raise the cub. They also intervene quickly and provide veterinary care at the first sign of sickness. Therefore, there is little variation in offspring survivorship in captive giant pandas once a cub is produced. This may be why our results with respect to offspring differed from Columbia Basin pygmy rabbits (Martin \& Shepherdson 2012) and house mice (Drickamer et al. 2000a; Drickamer et al. 2003; Gowaty et al. 2003). It is possible that 'hand-reared' and 'mother reared' is not a reliable proxy for offspring survivorship in giant pandas in these breeding situations and that future studies should consider tracking cub survivorship and reproductive performance into adulthood to get more accurate measurements of offspring fitness. Our findings showing increased reproductive performance measurements when individuals are mated to preferred mates are also consistent with the results of studies on mice (Mus domesticus) (Drickamer et al. 2000b; Drickamer et al. 2003), fruit flies (Drosophila pseudoobscura) (Anderson et al. 2007), wild guinea pigs (Galea musteloides) (Hohoff et al. 2003), pigeons (Columba livia 
domestica) (Klint \& Enquist 1981), pipe fish (Syngnathus typhle) (Sandvik et al. 2000), and kestrels (Falco punctatus) (Jones et al. 1995).

Sexual selection has been cited as a major force of evolution since Darwin postulated that mate choice confers an immediate advantage to the preferred individuals (Darwin 1859). Mate choice is believed to be an important evolutionary force in species survival and conservation because it influences the level of genetic variation maintained within populations (Carson 2003) and can also affect a population's risk of extinction (Kokko \& Brooks 2003; Moller 2003). From our study it appears that both male and female pandas discriminate between sexual partners and actively engage in choice and these preferences result in differential fitness to preferred individuals. These findings could have large ramifications for the captive population and current breeding program methods. In the current captive breeding system, pandas have limited opportunity for mate choice, and this may be an important factor in successful copulations and subsequent cub production.

Our findings are in agreement with Peng et al. (2009), the only other study on giant pandas that evaluated mate preference in captivity. Peng et al. (2009) found increased likelihood of intromission and cub production when both male and female giant pandas showed high levels of courtship behavior toward each other prior to mating versus when both or one of the pandas showed low levels of courtship behavior. They concluded that the level of courtship exhibited by a pair could be inferred as mate choice, however, this study did not use dichotomous choice tests or present two opposite sex conspecifics at once as we did in our study. In addiont, our study elaborates on Peng et al.'s (2009) findings and suggests that even pairs where one partner had low courtship frequencies 
(unpublished data) and/or giant panda pairs were composed of one nonpreferred partner could result in a successful pregnancy. We explicitly tested the preference of individual pandas for two opposite sex individuals which may be why our findings are slightly different from Peng et al. (2009). In addition, our analyses allowed us to compare four possible combinations of preferred and nonpreferred mate partners in our mutual mate preference experiments; P-P, NP-P, P-NP, and NP-NP.

Our mutual mate preference experiments indicated that preferred-preferred (P-P) pairings were the most successful pairings, followed by mate pairings where either the male or the female preferred their mate but preference was not reciprocated (NP-P and PNP pairings). Nonpreferred-nonpreferred (NP-NP) pairings conferred no reproductive benefits. Our findings are similar to Drickamer et al. (2003) in house mice and Anderson et al. (2007) in Drosphilia. Though Drickamer et al. (2003) did not find higher litter production rates in house mice they did find enhanced fitness for the P-P pairings compared to NP-NP in the number of pups weaned, birth-to-weaning viability and offspring mass. Our mutual mate preference studies specifically evaluated whether male or female preference was more predictive of reproductive performance measurements. Intromission success seemed to be determined more by male preferences as NP-P mate pairings, where the female did not prefer the male but the male preferred the female, showed slightly higher intromission success than P-NP or NP-NP mate pairings (though the difference was not significant). This trend also seems to be carried through to cub production, though, again, it was not statistically significant. We suspect a larger sample size is needed to definitively conclude that mate pairings where males preferred females but not vice versa have higher cub production than NP-NP and P-NP pairings. 
Our results suggest that breeding managers should consider providing male and female giant pandas with mate choice and determine mate preference prior to introductions in order to maximize the reproductive output of the captive breeding program. We also suggest that follow-up studies should investigate male preference more thoroughly and delve into social and physiological aspects associated with a male's motivation to breed with a particular female.

\section{Further factors affecting reproductive performance measurements}

\section{Familiarity}

Production of cubs in the past by a particular mate pair correlated with successful intromission and cub production for both male and female giant pandas. This result is consistent with the finding that mate familiarity with a female increases mating success and production of offspring (Fisher et al. 2003b; Martin \& Shepherdson 2012; Roberts \& Gosling 2004b). This finding implies that if breeding managers can get a pair to successfully produce a cub once they will be more likely to breed and produce cubs in the future. On the other hand, none of our familiarity measurements during the year leading up to the attempted mate pairings (i.e. familiarity status of potential partners in neighbor location for one year, one month, or in the weeks directly prior to breeding) predicted intromission success or cub production. These findings were surprising because for giant pandas it is thought that familiarity prior to breeding greatly increases reproductive performance and, therefore, breeding managers attempt to familiarize a pair directly prior to breeding by moving giant pandas to adjacent enclosures. Our study suggests that this intensive movement of giant pandas prior to breeding may not be necessary for increased reproductive performance. 
In light of the giant panda's natural history, lack of increased reproductive performance with familiarity may not be so surprising. In the wild, giant pandas locate estrous females that climb to the tops of trees while males compete nearby. It is thought that males visit multiple females' home ranges during the breeding season, the only time period during which females tolerate conspecifics in their home range (Nie et al. 2012c). In all observed mating congregations, the dominant male of the mating competition will secure breeding rights to the female, however, it is unknown if females ever reject dominant males and/or mate with subordinates. To our knowledge, there is only one study that investigated giant panda male movements prior to breeding competitions (Nie et al. 2012b). Radio collared males in this study were found in mating aggregates within their home range and, thus, were probably males that were familiar with the estrous female. However, the radio collared males were never the dominant males in the mating aggregate. Familiarity of the remaining males was unknown and it could not be determined if these males traveled into the female's home range (and would be unfamiliar with females) or were in adjacent home ranges most of the year (Nie et al. 2012b). Thus, although we can say the familiarity of the male did not necessarily confer any reproductive benefit to the mate pair, we cannot rule out the possibility that familiarity did confer a benefit (i.e. if dominant males were familiar). Our findings suggest that familiar males in mating congregations would only have a reproductive advantage if they had previously produced a cub with the female. Detailed studies of wild male panda movements prior to the breeding season linked with genetic parentage assignments may help further elucidate the effect of familiarity on reproductive performance in the giant panda. 


\section{Morphology}

This study was not designed to control for the morphological characteristics that may determine mate preference. However, we did find significant effects of both morphology and breeding history that may inform breeding managers and future studies. For female intromission success we found no relationship between most of the morphological and breeding history explanatory factors. However, female's mated to larger, older males were more likely to have intromission with that male. This may be due to increased sexual experience of older males. In addition, male-male competition frequently favors larger males which in turn is linked to increased fitness benefits in other species (Le Boeuf 1974; Poole 1989). In addition, it has been shown that female giant pandas have the capacity to perceive vocal signatures of different conspecifics that, in turn, appear to be related to variations in male body size (Charlton et al. 2009). In addition, White et al. (2002) found evidence that handstand scent signaling may have evolved as a signal of aggressive intent because of its relationship to body size, indicating that assessing body size is important to the species. In the wild, Nie and colleagues (2012b) found that dominant males were consistently ranked by observers as the largest male and would always secure breeding rights to the female. Thus, the current study lends further support to the suggestion that male size is an important factor in reproductive performance. Our findings are also consistent with studies in the wild on other species where female preference for large males and subsequent increases in reproductive performance have been linked in elephants (Poole 1989), barnacles (Hastings 1988), bullhead trout (Bisazza \& Marconato 1988), damselfish (Hoelzer 1990), Japanese medaka (Howard et al. 1998), and beetles (Harari et al. 1999). The increased 
likelihood of males producing a cub was also correlated with male mass. Studies specifically investigating morphological features and the production of high quality sperm are few, but Howard et al. (2006) present data from young males versus older males. Their data suggest that larger and older males produce more ejaculate volume with higher sperm concentration per milliliter than smaller, younger males (Howard et al. 2006). We could theorize that larger males might produce more cubs due to higher fertilization rates obtained from larger ejaculate volumes and more sperm per ejaculate, however, further data collection on semen quality of mating males would be needed to draw a direct conclusion. Alternatively, larger males may be able to hold intromission postures for longer increasing fertilization rates or postcopulatory female physiological mechanisms favoring fertilization, implantation, and embryo survival may be enhanced for females mated to a preferred male.

\section{Past breeding experience}

We found mother reared females had significantly more cub production than females that were hand reared. This finding suggests that behavioral development may differ between hand-reared and mother-reared females with an ultimate impact on later reproductive performance when those females raise their own cubs. Snyder et al. (2003) found that hand-reared giant panda cubs were behaviorally deficient in social skills compared to mother-reared cubs. Thus, we suggest that females properly socialized by their mother may have a better capacity to produce cubs compared to hand-reared counterparts. Further investigation into how hand-rearing and mother-rearing may alter reproductive performance measurements should be conducted.

Male cub production was significantly correlated with male birth location. Males 
born in the wild produced more cubs than males born in captivity. This finding is in agreement with previous experiments that found increased breeding success of giant pandas that were wild born (Ellis et al. 2006; Lindburg et al. 1998). Ellis et al. (2006) and Snyder et al. (2003) suggest that this difference in captive versus wild males may ultimately be linked to hand-rearing versus mother-rearing of individuals. As suggested above, we recommend that future studies increase sample sizes of wild and captive born males while controlling for male mass and age to clarify relationships with reproductive performance.

\section{Final Conclusions}

The role of mate preference and familiarity in reproductive performance of the giant panda has been demonstrated experimentally through our current study. Our results are in agreement with previous findings of mate preference in endangered specie (Martin \& Shepherdson 2012), and for the giant panda demonstrates practical importance for exsitu breeding programs. These findings may be applicable to many of the endangered species currently maintained in captive-breeding programs that have low reproductive performance. Constraining choice may, at the worst, deter individuals from breeding (Møller \& Legendre 2001), or may force animals to mate with low quality males, suffering reduced genetic and direct benefits, lowered offspring quality (Drickamer et al. 2003), and a reduction in fecundity and offspring investment (Quader 2005). We suggest that managers incorporate behavioral measurements of mate preference, as well as following genetic guidelines, to determine acceptable mate pairings. Both factors are important for ex-situ breeding of endangered species. 


\section{Acknowledgments}

We thank the CCRCGP giant panda keepers, researchers and staff for all their help and support. This work was supported by an Animal Behavior Society Student Research Grant, an Oregon Zoo Future for Wildlife Grant, and a Portland State University's Forbes Lea Grant.

\section{Supporting Information}

GLMMs of female giant panda fitness measurements of intromission success (Appendix A, Table 1) and cub production (Appendix A, Table 2) and female mate preference (Appendix A, Table 3) are available at the end of this dissertation. GLMMs of male giant panda fitness measurements of intromission success (Appendix B, Table 1) and cub production (Appendix B, Table 2) are available at the end of this disseration. The authors are solely responsible for the content and functionality of these materials. Queries (other than absence of the material) should be directed to the corresponding author. 
Table 2.1. Measures of reproductive performance for mate pairings of female giant pandas.

\begin{tabular}{lcccc} 
& \multicolumn{4}{c}{ Pairing Type* } \\
\cline { 2 - 5 } Variable & Previous Litter & No Previous Litter & Preferred & $\begin{array}{c}\text { Non- } \\
\text { preferred }\end{array}$ \\
\hline Total mate pairings & 10 & 34 & 25 & 16 \\
Total successful intromissions & 10 & 16 & 18 & 5 \\
Cubs produced & 9 & 11 & 16 & 2 \\
Mother reared cubs & 8 & 8 & 13 & $1(0.71)$ \\
Mean number of cubs & $1.2(0.38)$ & $1.5(0.47)$ & $1.4(0.28)$ & $10.5(2.63)$ \\
Mean male age (years) & $13.5(4.08)$ & $11.7(2.00)$ & $13.2(2.63)$ \\
Mean female age (years) & $12.7(3.83)$ & $10.2(1.76)$ & $11.1(2.22)$ & $10.7(2.67)$ \\
Mean male body mass $(\mathrm{kg})$ & $126.6(38.18)$ & $111.0(19.04)$ & $115.8(23.16)$ & $112.4(28.11)$ \\
Mean female body mass $(\mathrm{kg})$ & $108.9(32.83)$ & $110.55(18.96)$ & $109.7(21.93)$ & $112.7(28.17)$ \\
\hline
\end{tabular}

* Parentheses are standard errors 
Table 2.2. Measures of reproductive performance for mate pairings of male giant pandas.

\begin{tabular}{lcccc} 
& \multicolumn{4}{c}{ Pairing Type $^{*}$} \\
\cline { 2 - 5 } Variable & Previous Litter & No Previous Litter & Preferred & $\begin{array}{c}\text { Non- } \\
\text { preferred }\end{array}$ \\
\hline Total mate pairings & 11 & 37 & 24 & 16 \\
Total successful intromissions & 10 & 16 & 18 & 5 \\
Cubs produced & 8 & 11 & 14 & 3 \\
Mother reared cubs & 8 & 8 & 13 & 2 \\
Mean number of cubs & $1.3(0.44)$ & $1.6(0.49)$ & $1.4(0.38)$ & $1.7(0.96)$ \\
Mean male age (years) & $13.7(4.14)$ & $11.2(1.83)$ & $12.0(2.46)$ & $11.3(2.83)$ \\
Mean female age (years) & $13.2(3.97)$ & $10.6(1.74)$ & $11.1(2.26)$ & $11.1(2.78)$ \\
Mean male body mass $(\mathrm{kg})$ & $127.8(38.54)$ & $112.6(18.51)$ & $124.1(25.32)$ & $113.3(28.31)$ \\
Mean female body mass $(\mathrm{kg})$ & $110.4(33.28)$ & $110.26(18.12)$ & $110.2(22.50)$ & $110.7(27.68)$ \\
\hline
\end{tabular}

* Parentheses are standard errors 


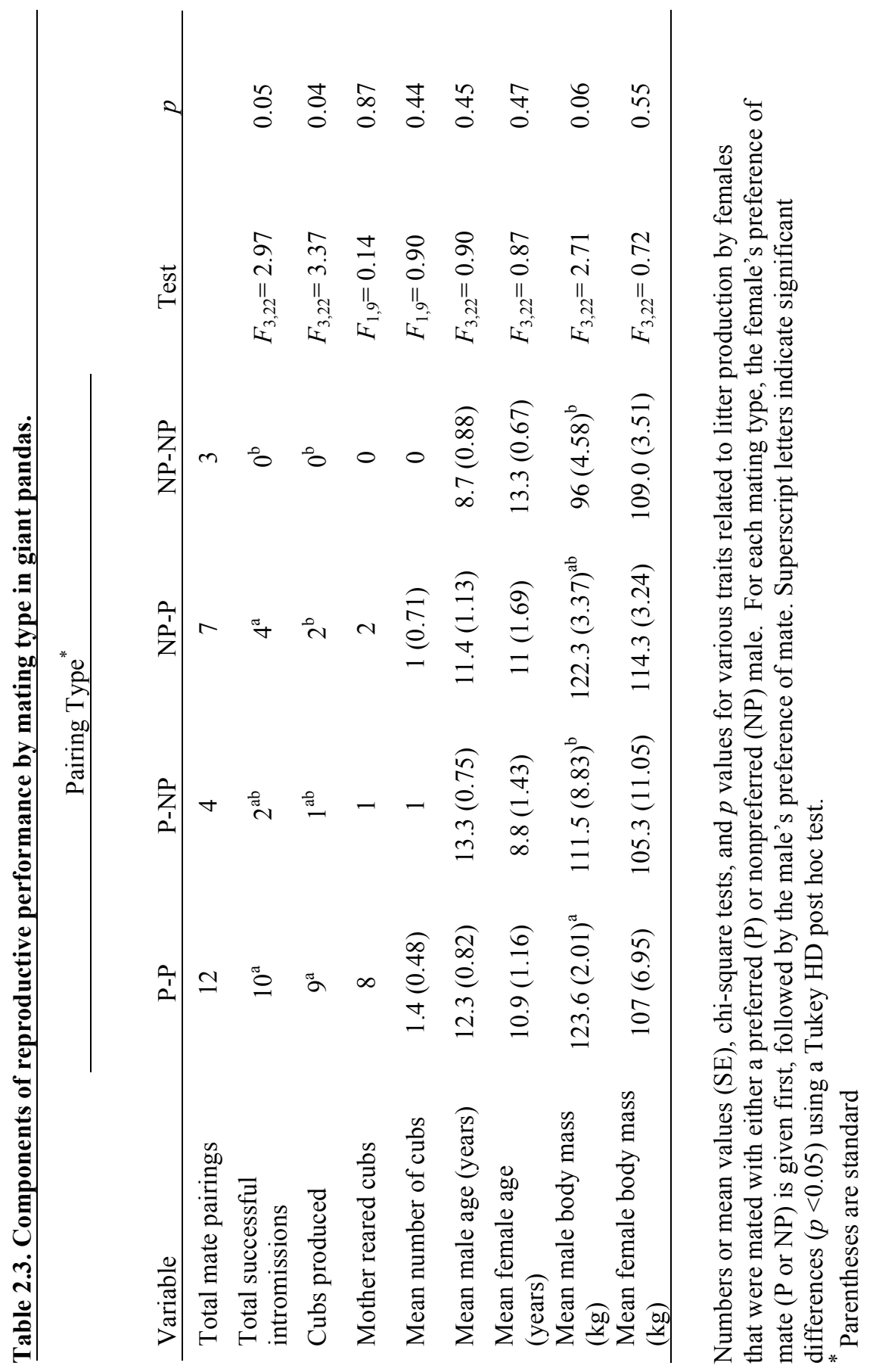




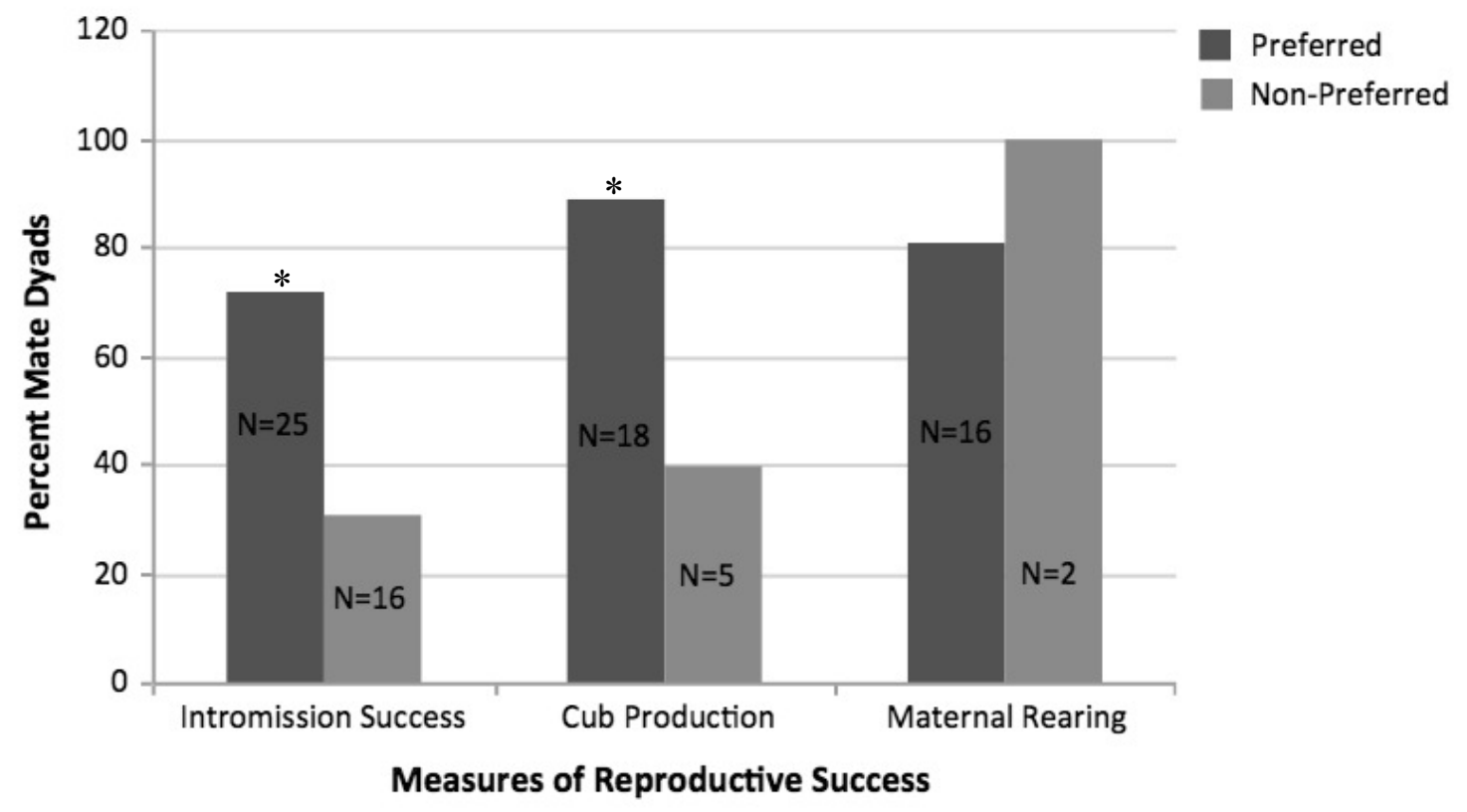

Figure 2.1. Percentage of mate dyads for females' preferred and nonpreferred mates grouped by intromission success, cub production, and maternal rearing for giant pandas. Dark bars represent dyads where the female was mated to a preferred male and light bars represent dyads where the female was mated to a nonpreferred male.

* indicates $p \leq 0.05$ for t-test. 


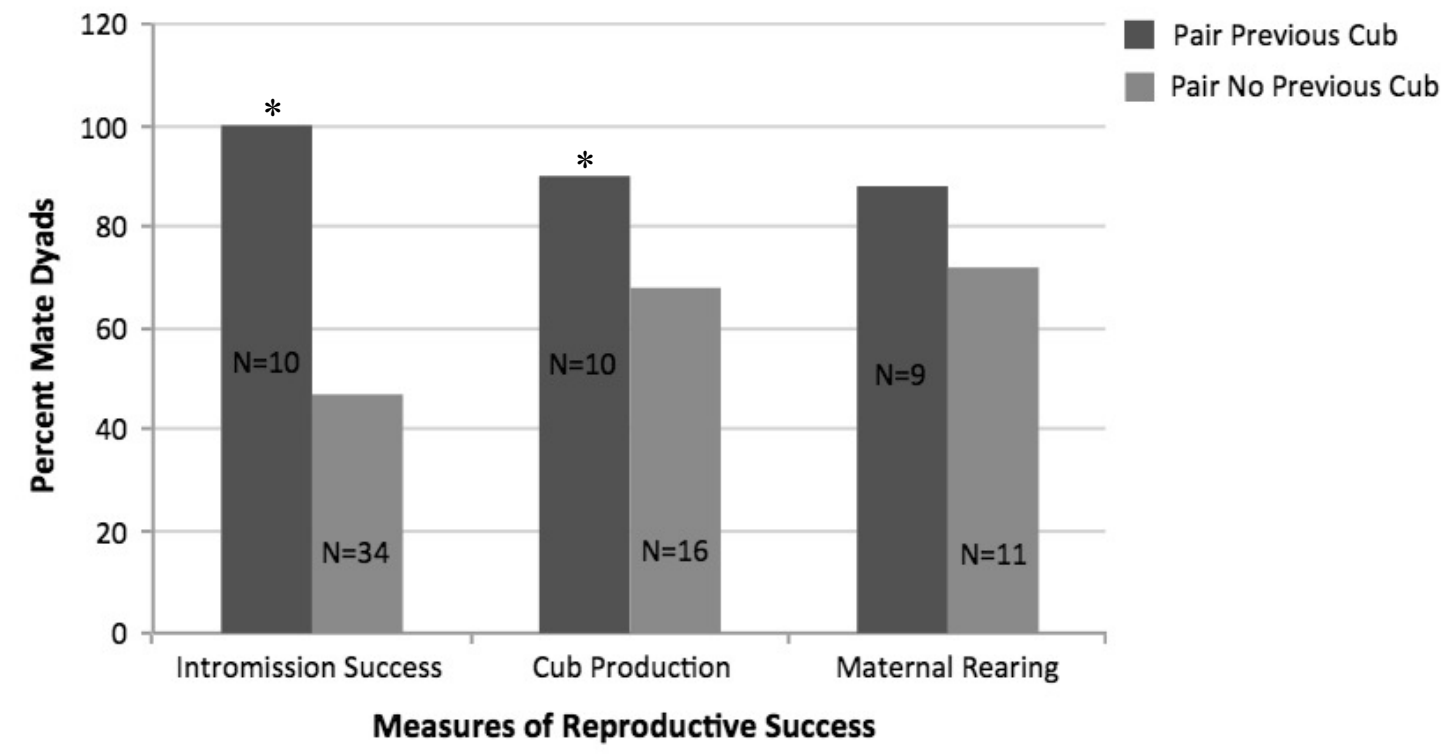

Figure 2.2. Percentage of mate dyads for females' mated with males that had previously sired one of their cubs and males that had not grouped by intromission success, cub production, and maternal rearing for giant pandas. Dark bars represent dyads where the female had previously produced a cub with that male and light bars represent dyads where the female had not previously produced a cub with that male. * indicates $p \leq 0.05$ for t-test 

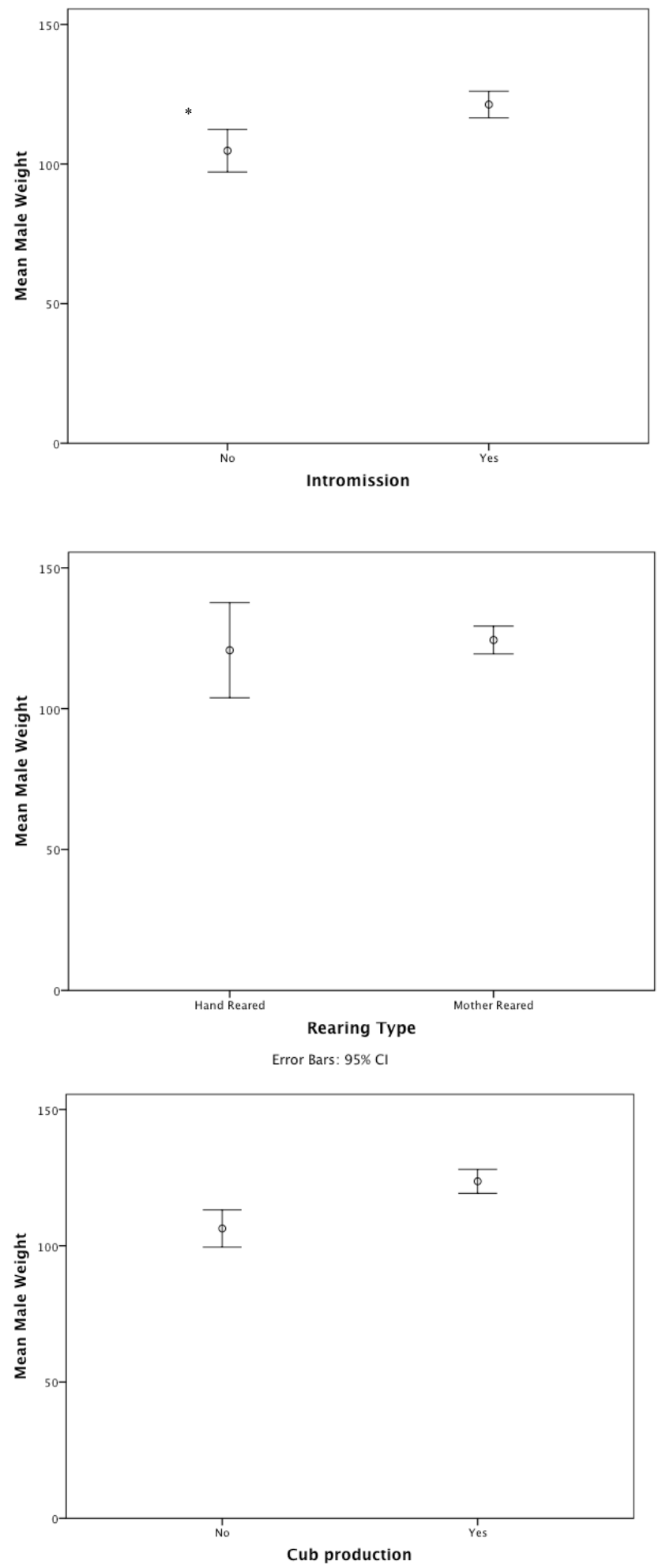

Figure 2.3. Mean mass of a) males introduced to females that had successful intromission and females that did not have successful intromission, b) males introduced to females that produced cubs and females that did not produce cubs, c) males introduced to females that reared their cubs and females that did not rear their cubs. * indicates $p \leq 0.05$ for t-test. 


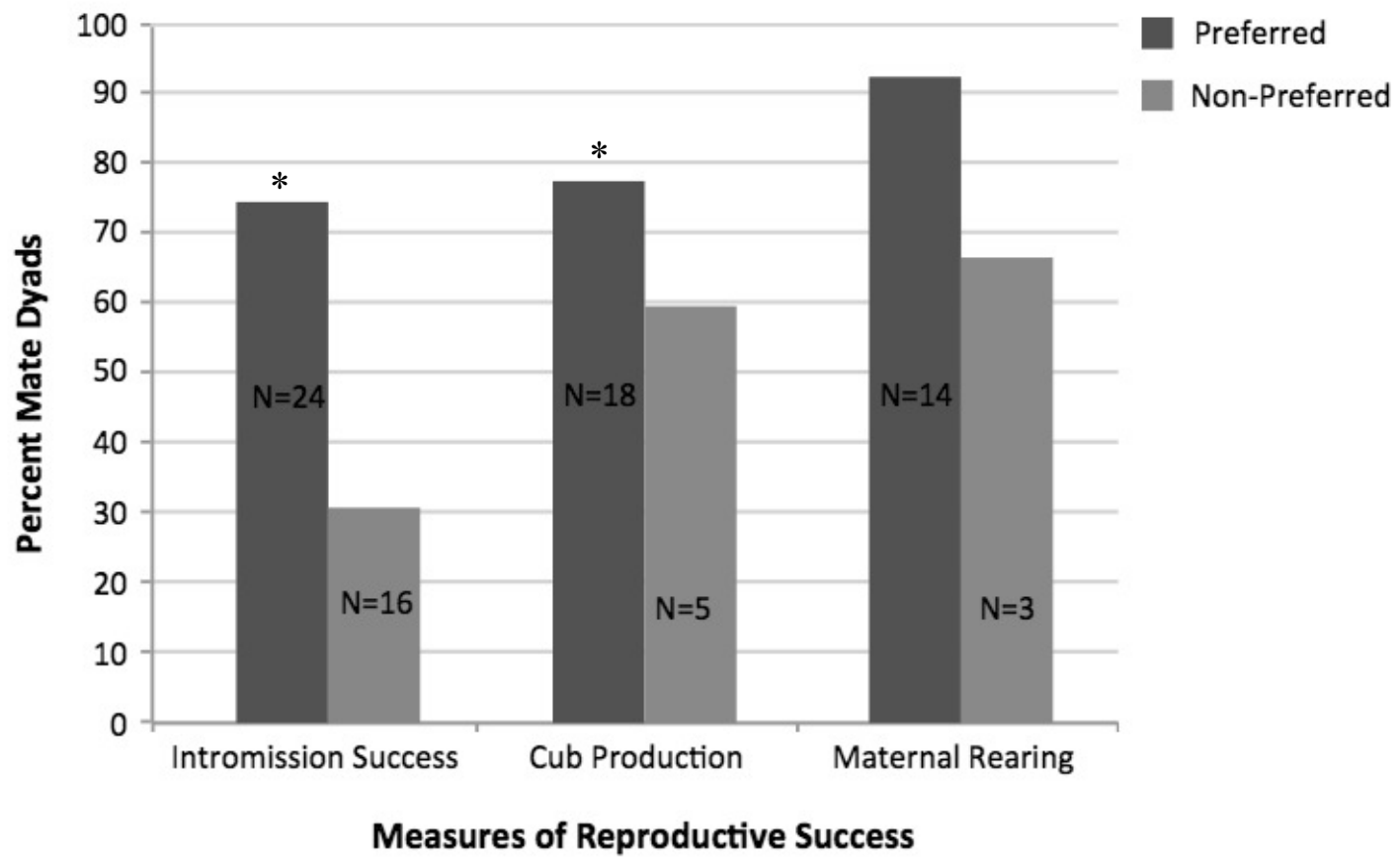

Figure 2.4. Percentage of mate dyads for males' preferred and nonpreferred mates grouped by intromission success, cub production, and maternal rearing for giant pandas. Dark bars represent dyads where the male was mated to a preferred female and light bars represent dyads where the male was mated to a nonpreferred female. * indicates $p \leq 0.05$ for t-test. 


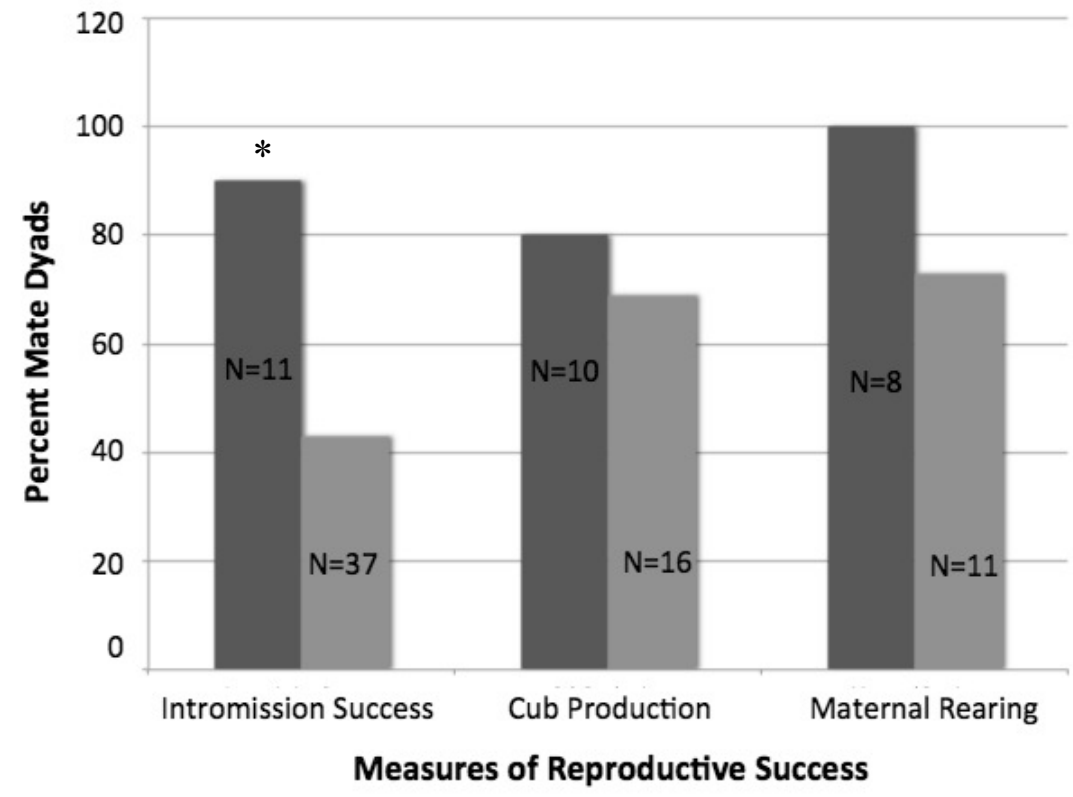

Pair Previous Cub

Pair No Previous Cub

Figure 2.5. Percentage of mate dyads for males' mated with females that had previously sired one of their cubs and females that had not grouped by intromission success, cub production, and maternal rearing for giant pandas. Dark bars represent dyads where the male had previously produced a cub with that female and light bars represent dyads where the male had not previously produced a cub with that female. * indicates $p \leq \mathbf{0 . 0 5}$ for t-test. 


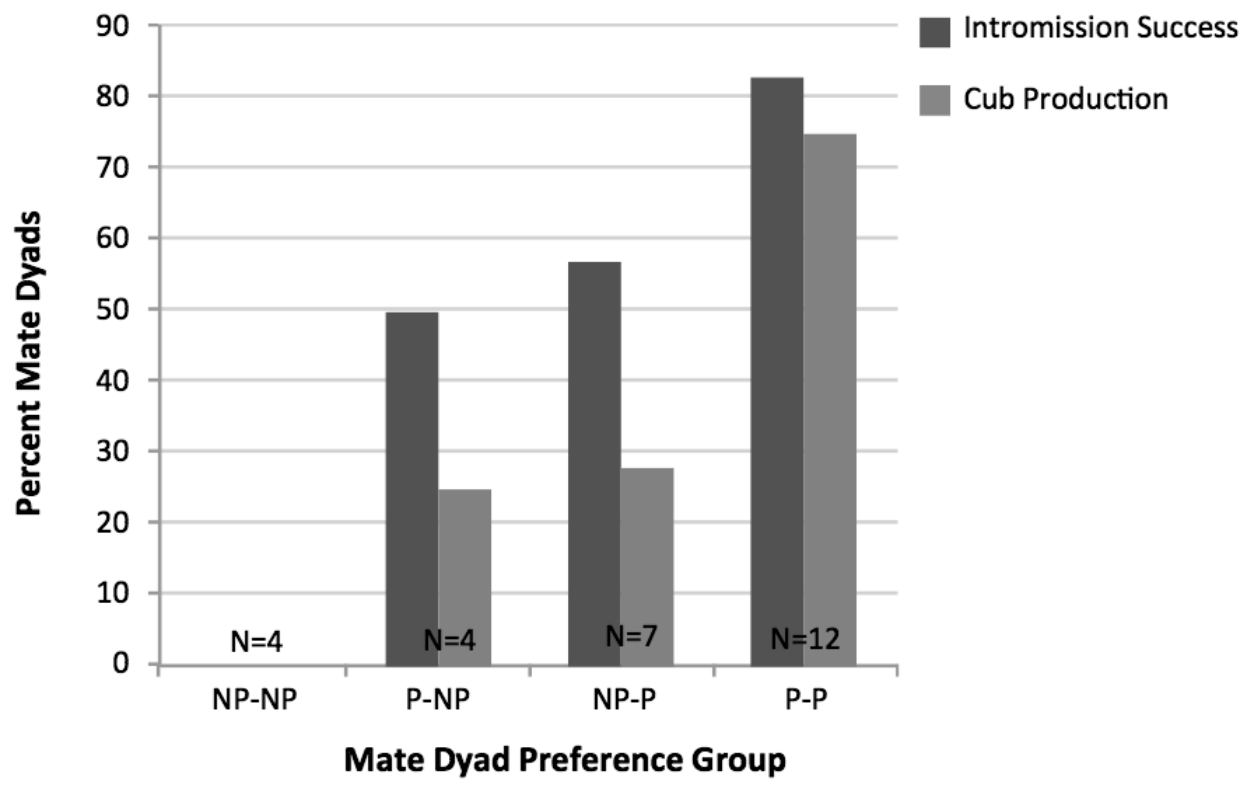

Figure 2.6. Percentage of mate dyads for different combinations of male and female preference; male does not nonpreferred and female is nonpreferred (NP-NP), female prefers male but male does not prefer female (P-NP), female does not prefer male but male prefers female (NP-P), and both animals prefer each other. Dark bars represent intromission success and light bars represent successful cub production. $\mathrm{N}$ indicates number of dyads total in the group. * indicates $p \leq 0.05$ for ANOVA. 


\section{CHAPTER 3: PERSONALITY AS A PREDICTOR OF LONG-TERM REPRODUCTIVE PERFORMANCE IN CAPTIVE GIANT PANDAS}

Running head: Personality and reproductive performance in giant pandas

Keywords: personality, temperament, reproductive performance, captive breeding, giant panda

Word Count: 8,291

Page Count: 29 


\section{Abstract}

Recently, personality of individual animals has been implicated as a factor in the failure or success of captive management and breeding of threatened and endangered species. Unintentional domestication through selection of personality types that may thrive in captive environments may lead to differential genotypes/phenotypes that diverge from those seen in natural populations. We investigated the role of personality on reproductive performance of giant pandas from mating behaviors through cub production. Measurements of personality included novel object tests and keeper surveys; both measures significantly correlated with variation in reproductive performance measurements. PCA of keeper surveys resulted in four components above the elbow of the scree plot that accounted for $53.3 \%$ of the observed variance in giant panda personality. These components were labeled according to the variables showing the highest positive loading: 'aggressive' (component I), 'playful-clever' (component II), 'fearful' (component III), 'stereotypical-excitable' (component IV). Our results show that for both male and female giant pandas, high 'fearful' individuals had lower natural mating success and cub production than low 'fearful' individuals . In addition, 'aggressive' female but not male giant pandas had increased natural mating success but decreased cub production. There was a positive correlation between a female giant panda's frequency of positive mating behaviors and having a high 'aggressive' component but a negative correlation with the raw keeper rating of 'fearful of conspecifics'. For males, the frequency of positive mating behaviors was negatively correlated with the raw keeper rating of 'fearful of conspecifics' and with the raw keeper rating of 'playful-clever' component. We stress the importance of considering husbandry 
practices to accommodate personality traits and ultimately increase reproductive performance in the captive population while simultaneously maintaining both genetic and behavioral diversity. 


\section{Introduction}

Conservation breeding to restore and/or supplement wild populations is an increasingly important tool for the recovery of threatened and endangered species. However, efforts to create self-sustaining populations of endangered species through captive breeding have been disappointing. Lees and Wilcken (Lees \& Wilcken 2009b) analyzed 87 mammal populations in zoos and found that $52 \%$ were not breeding to replacement and that $67 \%$ fell below the threshold of 200 animals recommended to sustain genetically healthy populations by Baker (2007). In captive-breeding programs, breeding pairs are typically chosen on the basis of minimizing inbreeding and maintaining founder representation. Though these goals are widely recognized as important, they have emphasized pairings based on genetic suitability largely at the expense of behavioral compatibility (Martin \& Shepherdson 2012; Rabin 2003; Swaisgood \& Schulte 2010). A potential consequence of captive breeding is unintentional domestication wherein the captive environment selects for particular genotypes/phenotypes divergent from those seen in natural populations (Christie et al. 2012). For example, in steelhead salmon, Christie et al. (2012) showed that there was a clear trade-off to successful reproduction in captivity and survival in the wild. Fish that successfully bred in captivity produced offspring that performed poorly in the wild, however, wild bred fish produced offspring poorly in captivity but had higher offspring survivorship in the wild (Christie et al. 2012). Selection on genetically heritable traits has been shown to result in rapid evolution in captive-breeding and reintroduction programs (Christie et al. 2012; Stockwell et al. 2003) as well as in the wild (Stockwell \& Ashley 2004), a phenomenon often referred to as "domestication." The idea of captivity 
domesticating wild animals and altering their behavior has been discussed in the scientific literature since Darwin (1859) introduced it in the "Origin of Species". Subsequently, scientists such as Flannery et al. (1969) and Rindos and Dunnell (1984) have elaborated on the mechanisms and consequences of domestication, but recently these areas of research have taken on a new significance as the number of endangered species continues to rise alarmingly and captive breeding as a recovery tool becomes more common. To our knowledge, only four species bred in captivity, steelhead salmon (Oncorhynchus mykiss), the oldfield mouse (Peromyscus polionotus subgriseus), Mallorcan midwife toad (Alytes muletensis), and the large white butterfly (Pieris brassicae), have been shown to change physical or behavioral traits or fecundity with multiple generations in captivity (Christie et al. 2012; Elsbeth McPhee 2004; KraaijeveldSmit et al. 2006; Lewis \& Thomas 2001). All of these are small, short-lived species. Strong tests of domestication on large, long-lived endangered mammals have not been conducted even though many of these species are bred in captivity with plans for reintroduction into the wild.

Personalities (or temperaments) are defined as consistent dispositions that modulate behavior. They are evolutionarily labile phenotypic traits, strongly linked to fitness, and known to be sensitive to anthropogenic alterations to the environment (McDougall et al. 2006; Réale et al. 2007; Sih et al. 2004). In captive environments, dramatically altered selective regimes (e.g. environmental effects of captive rearing, inbreeding, relaxed natural \& sexual selection, unintentional domestication selection) could inadvertently favor personalities pre-adapted to captive environments (for example, perhaps bold individuals have higher reproductive fitness in captivity). As a consequence, natural 
selection or unintentional domestication selection can potentially change the relative frequencies of temperament traits and possibly result in the loss of temperament diversity. As these are often linked to morphological and physiological traits, many conservationists are sounding the alarm (McDougall et al. 2006). Behavioral diversity that is reflected in personality diversity represents the potential for a population to thrive in changing environments and thus a reduction in personality types may lead to reduced adaptability and evolutionary potential (Conrad et al. 2011; McDougall et al. 2006). For aspects of personality that are heritable, captive environments, through intense selection, could lead to the rapid loss or alteration of personality types. A growing body of research indicates that personality is often genetically linked; for example, dominance in chimpanzees, (Weiss et al. 2002; Weiss et al. 2000); exploratory tendencies in great tits, (Dingemanse et al. 2002); boldness in squid, (Sinn et al. 2006); neophobia in horses, (Hausberger et al. 2004); fear in Japanese quail, (Jones et al. 1991); tameness in farmed fox, (Trut 1999); and emotionality in humans, (Jocklin et al. 1996)). For selection to occur, these traits must be both genetically inherited and have differential fitness outcomes (Réale et al. 2007)

Links between personality and fitness in the wild have been shown in yellowbellied marmots (Nowicki \& Armitage 1979), bighorns (Réale et al. 2000), and great tits (Dingemanse et al. 2004). Smith and Blumstein (2008) compiled data from multiple species to provide the first evidence that bold, aggressive individuals are more reproductively successful across species in both captivity and the wild, indicating that personality traits may have an evolutionary basis. Individual differences in behavior (i.e. personality profiles) have also been implicated in the failure or success of captive 
management and breeding of animals in zoo settings (Carlstead et al. 1999a; Carlstead et al. 1999b; Gold \& Maple 1994; Mendl et al. 1992; Powell \& Svoke 2008). For example, reproductive performance has been associated with fearfulness in cheetahs (Wielebnowski 1999) and dominance in black rhinoceroses (Carlstead et al. 1999b). Captive-breeding and the various selection pressures that are associated with it (i.e. natural, sexual, and artificial selection) could affect the expression of personality traits that may be critical to eventual reintroduction success (McDougall et al. 2006).

Giant pandas (Ailuropoda melanoleuca) have been notoriously difficult to breed in captivity and though research has increased the reproductive performance of the program in recent years, much is still unknown about the factors responsible for reproductive success and failure. It is frequently the case that only some individuals within the population breed, particularly for male giant pandas, and others fail to reproduce. Thus, investigation into the individual behavioral differences resulting in reproductive failure is a worthwhile endeavor and previous research on giant pandas indicates that personality factors may play a role in giant panda breeding. Zhang et al. (2004a) suggested that "an interaction between temperament and husbandry practices has played a major role in isolated cases of reproductive failure in female [giant pandas]" and reported that females with the most timid dispositions also experienced the greatest reproductive difficulties. Using keeper surveys, Ellis and colleagues (2006) found that aggressive individuals had higher reproduction but they combined natural mating, cub production, and litter survivorship to obtain four categories of breeding success that individual pandas could be grouped into; prime breeders, potential breeders, questionable breeders, and poor breeders. This method assumes that the same selective pressures are acting on each stage 
of reproductive performance and risks losing specificity. A recent study performed on giant pandas by Powell and Svoke (2008) found compelling evidence to suggest that zookeeper personality surveys do indeed correlate with novel object tests but they failed to establish statistically significant correlations with their small sample size $(N=4)$. A concurrent study conducted by this group suggested that bold male and female pandas showed less aggression towards mating partners (Powell et al. 2008). However, this study did not follow reproductive performance from mating behaviors through to the production of cubs so actual fitness benefits to personality traits could not be determined.

Building on these earlier studies, we investigated the role of personality on reproductive performance of giant pandas from pre-mating and mating behaviors through cub production. We pooled individual behavioral measurements in captive giant pandas assessed through novel object tests and animal keeper questionnaires to personality-type individual pandas. We sought to determine whether personality types correlated with measures of long-term reproductive performance.

\section{Methods}

\section{Study Site and Species}

Ages of giant pandas ranged from 6 to 18 years. Individuals were sexually mature and had been introduced to a member of the opposite sex on at least three different occasions for mating purposes to ensure that we were analyzing individuals who had been presented with the opportunity to mate. Housing and husbandry procedures for the breeding season can be found in Chapter 2. During the non-breeding season when personality tests were performed, animals are housed in large, naturalistic enclosures 
consisting of an open-air, naturally lit outdoor area (on average approximately $100 \mathrm{~m} \mathrm{x}$ $60 \mathrm{~m})$ and an indoor area $(3 \mathrm{~m} \times 5 \mathrm{~m})$ which animals have free access during all hours except for during cleaning. The outdoor enclosures have large, man-made, wooden structures and trees for climbing, ponds with water, and PVC pipes embedded in the ground for natural, upright bamboo presentation. The outdoor enclosure walls are $4.5 \mathrm{~m}$ tall concrete walls. Enclosures varied in the amount of physical and chemical contact individual giant pandas had with conspecifics ranging from no contact ability to barred windows between enclosures allowing limited physical and chemical contact. Animal care and use guidelines of the American Society of Mammalogists (Animal Care and Use Committee 1998; Assurance \#: A3675-01) were followed by all facility operators.

\section{Personality Typing}

We conducted personality studies on 22 female and 11 male giant pandas during the non-breeding season (May-October) of 2012 and 2013. We assessed personality of giant pandas in two ways; 1) zookeeper personality surveys and 2) novel object tests. Personality has traditionally been assessed through direct observations carried out by one observer or by various observers that have been trained and tested for inter-observer reliability. Though many scientists would claim that subjective animal keeper surveys are unreliable, recent research has shown that these surveys can predict real and important phenomena (Carlstead et al. 1999a; Carlstead et al. 1999b) and have been proven as a validated form of assessing animal personality (Carlstead et al. 1999b; Momozawa et al. 2003; Powell \& Svoke 2008; Watters \& Powell 2012; Wielebnowski 1999). The most commonly used behavioral test is the "novel object/situation test". These tests measure animal responses to some form of novelty (exposure to novel environments, stimuli, 
conspecifics, humans) or to stimuli that are known to be stressful (Powell \& Svoke 2008; Shepherdson et al. 2013; Wielebnowski 1999). Novel object tests are often used to assess personality/temperament because they directly measure an animal's response to a standardized environmental challenge and can be used to assess animal personality quickly (Marieke Cassia \& David 2012; Shepherdson et al. 2013). For this study, we modified and incorporated methodology from Wielebnowski (1999), Powell and Svoke (2008), and Shepherdson et al. (2013).

\section{Animal Keeper Surveys}

We conducted interviews with the primary keepers of each panda and had them complete a survey that included 23 behavioral adjectives that were rated on a 10-point Likert scale (Table 3.1). Before rating the pandas, the keepers were given an explanation of the rating procedure and the definitions of given adjectives. We considered the keeper who spent most time with the panda as the "primary" rater and used his/her responses in the data analyses. Keepers were instructed to score the panda compared to all pandas they have ever known.

\section{Novel Object Test}

Novel object tests were used to assess temperament and obtain objective behavioral data for comparison with keeper ratings. A total of four enrichment trials were conducted per panda using four different objects the pandas had not encountered before at the CCRCGP. These included: 1) ice blocks, 2) ice blocks with $1 / 2$ apple and $1 / 2$ carrot at the surface (fruitcicles), 3) large tubs of water with fruit and 4) a rubber ball with a diameter of $75 \mathrm{~cm}$. Ice blocks were large blocks of ice approximately $20 \mathrm{~cm}^{3}$. Fruit tubs were $75 \mathrm{~cm}$ $\mathrm{x} 60 \mathrm{~cm} \times 60 \mathrm{~cm}$ filled with water with one apple cut in half and one carrot floating in the 
water. Balls and tubs were washed in between trials and soaked in a bleach bath for 10 minutes to remove conspecific scents.

All trials were performed between 1330 and 1600 as this period was outside of morning and afternoon feeding times. Observations during this time period allowed a continuous hour of observation without interruption and could be held constant between pandas controlling for activity level changes throughout the day. Trials consisted of moving the panda into the indoor portion of the enclosure, removing all other food from the outdoor enclosure, setting up the novel object 2-3 meters away from the indoor enclosure door, and releasing the panda into the outdoor area to start the novel object trial. We scored panda behavior for one hour after introduction to the novel object. The indoor enclosure door remained closed throughout the entire behavioral scoring period. All trials were performed for each panda but no panda received more than one trial in a day (i.e. all pandas received trials over five days). Approximately $24-48$ hours prior to the enrichment presentation, we observed the panda for a one-hour control session with no enrichment present. Each control session occurred at the same time of day as the enrichment trials with which it was paired. Order of novel object presentation was randomly assigned with regard to trial order.

We used instantaneous point sampling at one-minute intervals to estimate the percentage of time spent in various activities. We recorded mutually exclusive behaviors according to an ethogram derived from Swaisgood and Owen (2007). Behaviors were then grouped into major behavioral categories (Table 3.2); including total number of different types of stereotypical behaviors, total time spent in stereotypical behavior, total point samples that were recorded under a stereotypical behavior, interacting with the 
novel object, performing active behaviors (e.g. climbing, locomoting, etc.), chemical communication behaviors, affiliative vocal communication behaviors, and aggressive vocal communication behaviors. In addition, we recorded the latency to touch the object, number of visits to the object, number of visits to the indoor enclosure door, total time spent in direct contact with the object, and total time sitting at the indoor enclosure door.

\section{Mating Procedure}

Here we define mate pairings as the introduction of a specific male to a specific female for the purpose of breeding. In the wild and captivity, breeding season for the panda is from February to May. We began assessing mate pairing of adult giant pandas in mid-February and continued until the end of April in 2012 and 2013 (we had two females enter estrus in May over the course of the study). The assignment of individuals to mate pairs was based on a captive breeding and genetic management plan determined by breeding managers and designed to maximize genetic health of the captive population. To determine female estrous status, urine samples of female giant pandas were collected via syringe from the cages of female giant pandas during the breeding season approximately three days a week until baseline levels rose and then samples were collected daily through peak estrus and hormone decline. Urine samples were immediately processed by the CCRCGP for estrogens and progestins by enzymeimmunoassay previously validated on urine (McGeehan et al. 2002). Samples were used to establish date of estrus.

Males were introduced to female pens for mating between 9:00 and 11:00 a.m. Mating sessions were defined as a pairing of one male and one female (a mating dyad) from the time males were introduced to females until males were removed from the pen. 
Mating sessions lasted on average 15.5 minutes but ranged from 3-75 minutes. If either animal's behavior was aggressive, animal care staff removed the male immediately to prevent injury or death. After a mating session, males were moved back to their enclosures and subsequently were placed with a different female until all females had been mated to their recommended males. This method resulted in females being introduced to 1-4 males and having on average 4 (but as many as 9) mating opportunities each breeding season. As a fail-safe, female pandas are often artificially inseminated in addition to natural breeding. If paternity was in question, the CCRCGP established the father using DNA obtained from hair samples and amplified utilizing the PCR to analyze microsatellite loci after the methods of Zhang et al. (1994). If paternity could not be established, mate pairings associated with the cub production were removed from analysis.

Mating behaviors were defined from Swaisgood and Owen (2007). All occurrences of these behaviors were recorded along with the identity of the panda performing the behavior. In addition, we recorded latency to contact, latency to mounting, latency to copulation, and total copulation time. Recording started as soon as females and males were given access to each other in the outdoor mating enclosures and ended once keepers attempted to separate the animals. If copulation occurred recordings were ended as soon as the male and female broke contact as female pandas become very aggressive at the termination of copulation. Behaviors were scored per mating dyad, which was defined as one male and one female giant panda paired for the purpose of mating. Because some individuals had more than one mating attempt, breeding behaviors were averaged for each individual to yield total number of mate behaviors, total number of positive mating 
behaviors, total number of negative mating behaviors, and frequency of mating behaviors.

\section{Breeding History and Physiological Measures}

We monitored several measures of past reproductive performance by consulting studbooks, veterinary staff, and husbandry records to determine whether individuals had previous natural mating experience (breeder versus non-breeder) and if animals had successfully produced cubs in the past. We only used information about cub production and natural breeding history if we could confirm natural insemination (not artificial) and paternity of the cub from breeding manager records and genetic tests. This reduced our sample size but allowed us to focus on normal reproductive behavior (Powell et al. 2008b). We collected the following information from all individuals: provenance (wild born versus captive born), rearing (mother-reared versus hand-reared), gender (male or

female), age (in years), and mass ( $\mathrm{kg})$. These factors were found to be significant factors in intromission success and cub production in a recent study on mate preference in giant pandas performed by our research group (Chapter 2).

\section{Data Analysis}

We tested differences between behaviors in different novel object trials with oneway ANOVAs. ANOVAs and post-hoc tests revealed significant differences between controls and all tests, but since the rubber ball novel-object test resulted in the most differences between control periods and other novel object tests and also had the most variation across individuals (see results), we used only rubber ball novel-object tests for further analyses. Principal component analysis was run on keeper surveys and behaviors from the rubber ball novel object test to reduce variables into a substantially smaller set 
of uncorrelated variables (Wielebnowski 1999). PCA was carried out using a Varimax rotation. Scree plots were examined and components above the elbow were retained for interpretation and were labeled according to the two variables that showed highest positive loading for this component.

All data were assessed for normality and homogeneity of variance and appropriate statistical tests were conducted. In case we were losing specificity in component scores, we also evaluated raw scores on behavioral events measured during the rubber ball novel object test. Raw scores and component scores were compared to the scores of questionnaire items obtained for each of the 33 giant pandas using Spearman rank-order correlation coefficients. Component scores of all individual giant panda were compared to the variables; gender, provenance (wild born versus captive born), rearing type (handreared versus mother-reared), breeding status (breeder versus non-breeder), and cub production status (produced cubs versus had not produced cubs). The effect of age and mass on breeding status and on component scores was examined using the following statistics: Mann-Whitney U-test (age, mass and breeding status, cub production) and Spearman rank correlations coefficient (age, mass and component scores).

To investigate which factors may be contributing most to the reproductive performance outcomes we analyzed personality characteristics using generalized linear mixed models (GLMM). The first response variable was successful natural breeding in the past (binary response variable: yes $=1$, no $=0$; generalized linear mixed model with logit link function). The second response variable was whether a giant panda had produced cubs in the past (binary response variable: yes $=1$, no $=0$; model with logit link function). The potential explanatory variables/factors that could affect the success of a 
mating attempt were: provenance (binary response variable: captive $=1$, wild $=0$ ), focal animal rearing (binary response variable: mother $=1$, hand $=0$ ), age (years), and mass (kilograms). In addition, we included the personality keeper ratings and component scores as potential explanatory factors. Due to small sample sizes and lack of power, we ran keeper scores and component scores in separate GLMM models with the natural history and physiological measure described above. We used mass as a proxy for size as length and height for shown to be highly correlated in our previous study (Chapter 2). We ran GLMM through a step-wise exclusion method in which the least significant predictor variable $(p \geq 0.05)$ was sequentially removed from the model until the final significant model was revealed (McCullagh \& Nelder 1989). Models were compared using penalized log likelihood scores (Quasi-Akaike's Information Criterion (A=QIC); Crawley 2005). Minimal models were checked by adding all terms back in, one by one, and examining model QIC scores for possible improvement in model fit.

Statistical significance was determined at the level of $p<0.05$ for all tests. All analyses were performed in SPSS 19.0 for Mac OS X.

\section{Results}

\section{Observer/Keeper Questionnaire}

PCA resulted in four components above the elbow of the scree plot that accounted for $53.3 \%$ of the observed variance (Table 3.3). These components were labeled according to the variables showing the highest positive loading: 'aggressive' (component I), 'playful-clever' (component II), 'fearful' (component III), 'stereotypical-excitable' (component IV). Component I showed high positive loadings on 'aggressive toward 
conspecifics', 'aggressive toward humans', and 'solitary'. It showed high negative loadings on 'friendly toward conspecifics', 'friendly toward humans', and 'shy' (Table 3.3). Component II showed high positive loadings on 'playful', 'self-confident', 'clever', and 'innovative' and high negative loadings on 'solitary' (Table 3.3). Component III showed high positive loadings on 'fearful of humans', 'fearful of conspecifics', 'not interested', and 'nervous'. It showed high negative loadings on 'curious', 'friendly towards humans', and 'self-confident' (Table 3.3). Component IV showed high positive loadings on 'stereotypical', 'excitable', 'vigilant' and 'nervous' (Table 3.3). All negative loadings for this variable were low.

\section{Novel-Object Tests}

PCA scree plots did not reveal an obvious elbow but resulted in five components above eigan values of 1 that accounted for $87.77 \%$ of the observed variance (Table 3.4). These components were labeled according to the variables showing the highest positive loading: 'stereotypical-active' (component I), 'door directed' (component II), 'object directed' (component III), 'chemical communication' (component IV), and 'shy' (component V). Component I showed high positive loadings on 'total active behaviors', 'total time spent in stereotypical behavior', and 'number of stereotypical point samples'. It did not show high negative loadings on any behaviors (Table 3.4). Component II showed high positive loadings on 'total time spent at the shift door' and 'total number of visits at the shift door' and did not have any high negative loadings (Table 3.4). Component III showed high positive loadings on 'total time spent with the novel object' and 'total number of object interactions' and did not have any high negative loadings (Table 3.4). Component IV showed high positive loadings on 'total number of scent 
marks' and 'total number of chemical communication point samples' and did not have any high negative loadings (Table 3.4). Component V showed high positive loadings on 'latency to touch and object' and 'total number of negative vocal communications' and did not have any high negative loadings (Table 3.4).

When all novel object tests were grouped together within an individual panda, we found significant differences between individual giant pandas in behavioral responses during novel object tests. Giant pandas showed differences between individuals in total number of different stereotypes performed $\left(\mathrm{F}_{30,120}=1.69, p=0.03\right)$, total time spent in stereotypical behavior $\left(\mathrm{F}_{30,120}=1.60, p=0.04\right)$, total number of stereotypical point samples collected over one hour $\left(\mathrm{F}_{30,120}=2.36, p=0.001\right)$, total number of point samples performing active behaviors $\left(\mathrm{F}_{30,120}=2.33, p=0.001\right)$, total number of aggressive vocal communications $\left(\mathrm{F}_{30,120}=1.62, p=0.04\right)$, latency to touch the object $\left(\mathrm{F}_{30,120}=1.44, p=\right.$ $0.06)$, total number of visits to the indoor enclosure door $\left(\mathrm{F}_{30,120}=4.65, p<0.001\right)$, total time sitting at the indoor enclosure door $\left(\mathrm{F}_{30,120}=3.6, p<0.001\right)$. There was no significant difference across pandas in total number of interactions with the object $\left(\mathrm{F}_{30,120}\right.$ $=0.87, p=0.66)$, total number of chemical communication point samples $\left(\mathrm{F}_{30,120}=1.35\right.$, $p=0.13)$ and total number of vocal communication point samples $\left(\mathrm{F}_{30,120}=0.93, p=\right.$ $0.57)$, and total time spent with the novel object $\left(\mathrm{F}_{30,120}=1.01, p=0.46\right)$.

ANOVAs were conducted to analyze differences in behavioral responses across novel objects. Ice blocks and ball novel objects showed the largest ranges across behaviors (Table 3.5). Ball novel objects were either first or second largest range for 10 of the 13 measured behaviors, ice blocks for 8 of the 13 , fruitcicles for 5 of the 13 , and water tubs with fruit for 5 of the 13 . There was significant differences between novel 
object test in total number of different stereotypes performed $\left(\mathrm{F}_{4,145}=2.36, p=0.05\right)$, total number of visits to the object $\left(\mathrm{F}_{4,145}=13.9, p<0.001\right)$, total number of interactions with the object $\left(\mathrm{F}_{4,145}=23.48, p<0.001\right)$, and total time spent with the object $\left(\mathrm{F}_{4,145}=\right.$ 17.58, $p<0.001)$. Tukey HD post hoc tests revealed total number of different stereotypical behaviors performed during the fruitcicle novel object test varied significantly from the control $(p=0.05)$. There were no other significant differences between other novel object tests in total number of different stereotypical behaviors $(p>$ 0.05). Tukey HD post hoc tests revealed total number of visits to the ball novel object was significantly different than the water tub with fruit $(p=0.04)$ and the fruitcicle $(p=$ 0.02). There were no other significant differences between other novel object tests in total number of visits to the novel object $(p>0.05)$. Tukey HD post hoc tests revealed total number of interactions with the ball novel object was significantly different from the water tub with fruit $(p<0.001)$ and fruitcicle novel objects $(p<0.001)$. There were no other significant differences between other novel object tests in total number of interactions $(p>0.05)$. Tukey HD post hoc tests revealed total time spent with the fruitcicle novel object was significantly different from the ball $(p<0.001)$, water tub with fruit $(p<0.001)$, ball $(p<0.001)$, and ice block $(p<0.001)$. There were no other significant differences between other novel object tests in total spent with the novel $\operatorname{object}(p>0.05)$.

Several personality characteristics from the keeper questionnaires correlated significantly with the direct measures of behavior obtained during the novel object test. Latency to touch the object was negatively correlated with 'vigilant' and 'stereotypical' keeper ratings and positively correlated with 'innovative' keeper ratings (Table 3.6). In 
addition, latency to touch the object was positively correlated with the 'aggressive' (I) component and negatively with the 'stereotypical-excitable' (IV) component (Table 6). Total number of visits to the novel object positively correlated with 'active' keeper ratings and negatively with 'calm' keeper ratings (Table 3.6). Total time spent with the novel object positively correlated with 'aggressive to conspecifics' and 'solitary' keeper ratings as well as the 'aggressive' component (Table 3.6). Total time spent at the indoor enclosure door negatively correlated with 'aggressive to people' and 'clever' keeper ratings and the 'aggressive' component (Table 3.6). Total number of chemical communication point samples was negatively correlated with 'vigilant', 'aggressive to people', 'stereotypical', and 'excitable' keeper ratings and with the 'fearful' component (Table 3.6). Total number of aggressive vocalizations negatively correlated with 'vigilant' keeper ratings (Table 3.6). No other correlations could be found between keeper questionnaire ratings, component scores, and novel object behaviors. We used the PCA components obtained from the keeper surveys to evaluate personality on reproductive performance.

\section{Breeding History and Physiological Measures}

There was a significant difference between breeders and non-breeders in age. Breeders showed an average age of $\bar{x} \pm \mathrm{SD}=13.71 \pm 4.16$ years $(\mathrm{n}=14)$ and nonbreeders of $\bar{x} \pm \mathrm{SD}=8.67 \pm 3.5$ years $(\mathrm{n}=18)(\mathrm{U}=95.5, p=0.002$; Figure 3.1). Comparison of component scores of breeders versus non-breeders showed significant differences for the 'fearful' component (III). Non-breeders were significantly more 'fearful' on component scores $(\bar{x} \pm \mathrm{SD}=0.35 \pm 1.16)(\mathrm{U}=90, p=0.009)$ than breeders ( $\bar{x} \pm \mathrm{SD}=-0.15 \pm 0.80$; Table 3.7, Figure 3.2). The significant differences in component 
scores of breeders and non-breeders held up even when female $(\mathrm{U}=77, p=0.011)$ and male scores $(\mathrm{U}=4, p=0.045)$ were examined separately. We ran correlations between the 'fearful' personality characteristic and age to further investigate the relationships between these variables. Age and the 'fearful' (III) component score showed a high positive correlation with older individuals showing more 'fearfulness' $\left(\mathrm{r}_{\mathrm{s}}=0.45, p=\right.$ 0.009; Figure 3.3). There was no significant correlation between breeder and nonbreeder status and the remaining component scores or the mass, provenance, or rearing of giant pandas (Table 3.7).

There was a significant difference in age between female giant pandas that produced cubs and females that did not. Animals that produced cubs showed an average age of $\bar{x} \pm \mathrm{SD}=13.8 \pm 4.4$ years $(\mathrm{n}=13)$ and animals that did not produce cubs showed and average age of $\bar{x} \pm \mathrm{SD}=7.6 \pm 4.0$ years $(\mathrm{n}=18)(\mathrm{U}=10.95, p=0.004)$. In addition, females that produced cubs were rated lower by keepers on 'aggressive toward conspecifics' $(\bar{x} \pm \mathrm{SD}=4.33 \pm 1.92)$ than females that did not produce cubs $(\bar{x} \pm \mathrm{SD}=$ $6.78 \pm 2.82)(\mathrm{U}=23, p=0.026$; Table 3.7, Figure 3.4a). Female giant pandas that produced cubs also scored significantly lower on 'curious' scores $(\bar{x} \pm \mathrm{SD}=4.75 \pm 1.96)$ than females that did not produce cubs (curious: $\bar{x} \pm \mathrm{SD}=7.568 \pm 2.07)(\mathrm{U}=19.5, p=$ 0.013; Figure 3.4b). In addition, females that produced cubs had lower 'fear towards human' scores $(\bar{x} \pm \mathrm{SD}=1.78 \pm 1.64)(\mathrm{U}=85, p=0.022)$ compared to females that did not produce cubs $(\bar{x} \pm \mathrm{SD}=3.58 \pm 1.78$, Figure $3.4 \mathrm{~b})$. Females that produced cubs were rated lower on the 'fearful' component score $(\bar{x} \pm \mathrm{SD}=-0.27 \pm 0.98$; Table 3.7, Figure $3.4 \mathrm{~d})$ and lower on the 'aggressive' component score $(\bar{x} \pm \mathrm{SD}=-0.17 \pm 0.44$; Table 3.7, Figure 3.4e). We further investigated correlations between the personality characteristics 
and age to elucidate relationships between these variables. Female age showed a significant positive correlation with the 'fearful towards humans' keeper rating $(r=0.54$, $p=0.01)$ and the 'fearful' component score (III) $(\mathrm{r}=0.52, p=0.02)$. These differences were not observed for males or when the sexes were combined which contrasts with Ellis et al. (2006). No other personality keeper rating scores, PCA component scores, provenance, rearing or mass were different between giant pandas that raised their cubs and giant pandas that did not.

Correlations between mating behaviors, raw personality keeper ratings, and personality PCA component scores revealed some significant relationships. "Positive mating behaviors" were defined as all affiliative behaviors seen between a mating pair (e.g. bleats, chirps, angiogenital investigation, tail-up, lordosis, etc.). "Negative behaviors" were defined as all non-affiliative behaviors seen between a mating pair (e.g. barking, growling, aggressive physical contact such as swiping, chasing, charging, etc.). When the sexes were combined, there was a negative correlation between frequency of positive mating behaviors and the keeper rating of 'friendly toward people' (Table 3.8). The frequency of negative mating behaviors displayed by a panda showed a positive correlation with age, 'vigilant' and 'curious' keeper ratings and a negative correlation with 'fearful of people' keeper ratings. Frequency of total mating behaviors was positively correlated with age, the 'vigilant' keeper rating and negatively correlated with 'fearful' of conspecifics and 'fearful of people'. When females were investigated separately from males, there was a positive correlation between the frequency of positive mating behaviors and the 'aggressive' component (I) but a negative correlation with 'fearful of conspecifics' (Table 3.8). A female's frequency of negative mating behaviors 
positively correlated with age, 'curious keeper ratings' and 'friendly toward people' rating (Table 3.8). A female's frequency of total mating behaviors showed a positive correlation with the 'friendly toward people' keeper rating (Table 3.8). For males, the frequency of positive mating behaviors was negatively correlated with 'fearful of conspecifics' and 'innovative' keeper ratings and with the 'playful-clever' component (II) (Table 3.8). There were no correlations with a male's frequency of negative behaviors and any of the personality characteristics rated by the keepers or expressed by the PCA components. However, the frequency of negative behaviors was correlated with male mass (Table 3.8). Larger males expressed higher frequency of negative mating behaviors. The frequency of a male's total mating behaviors negatively correlated with age, 'fearful of conspecifics', 'innovative' keeper ratings and the 'fearful' component (III) (Table 3.8). There were no significant correlations between panda provenance and rearing.

There was no significant difference in component scores of males and females, wild born versus captive born individuals, and mother-reared versus hand-reared individuals (Table 3.7). However, there was a trend toward females being more fearful than males $(\mathrm{U}=80, p=0.09)$, mother-reared animals being more playful than hand-reared $(\mathrm{U}=53$, $p=0.09)$, and wild-born individuals being more aggressive than captive born $(\mathrm{U}=73, p$ $=0.067)$. Mass did not differ between breeders and non-breeders $(\mathrm{U}=30.5, p=0.272)$. No significant correlation was found between age and the remaining component scores (component I: $\mathrm{r}_{\mathrm{s}}=-0.006, p=0.97$, component II: $\mathrm{r}_{\mathrm{s}}=-0.31, p=0.084$, component IV: $\left.\mathrm{r}_{\mathrm{s}}=-0.199, p=0.274\right)$.

GLMMs revealed that age was correlated with increased natural mating success. 
The final model implicated age as being the most significant factor explaining natural mating success with older individuals mating more $\left(\beta=-0.36\right.$, Wald Chi-Square $1_{1,32}=$ 7.80, $p=0.005, B I C=38.97$; Appendix C, Table C.1). GLMMs revealed that both age and the 'aggressiveness' component were correlated with increased cub production. The final model implicated age as being the most significant factor explaining cub production with older individuals producing more cubs $\left(\beta=-5.24\right.$, Wald $_{\text {Chi-Square }}, 31=3.32, p=$

0.14; Appendix C, Table C.2). The 'aggressive' component was the only other factor that significantly correlated with cub production $\left(\beta=2.24\right.$, Wald Chi-Square $_{1,31}=1.75, p=$ 0.19). Because neither variable weighted significantly, we reduced the final model to one explanatory variable and compared BIC scores. The final GLMM with only age included showed a worse fit (i.e. higher) BIC score but had a significant negative correlation with cub production $\left(\beta=-0.36\right.$, Wald $\left._{\text {Chi-Square }}, 31=0.14, p=0.008, B I C=28.8\right)$. The final GLMM with only the 'aggressive' component included showed a worse fit (i.e. higher) $B I C$ score and no significant correlation with cub production $(\beta=0.56$, Wald ChiSquare $\left._{1,31}=0.41, p=0.17, B I C=48.1\right)$. Both of these models were significantly different from the GLMM that included both terms as indicated by a difference in BIC scores greater than 10. Thus, age and the aggressive component together were the best predictors of cub production giving the model with the best fit.

\section{Discussion}

\section{Relationships between keeper surveys and novel object tests}

We found significant variation in response of giant pandas to novel objects indicating that pandas vary in their response to novel stimuli. Because these behavioral 
responses correlated with keeper questionnaire ratings this indicates that novel object tests and keeper ratings provide a valid method for assessment of individual distinctiveness in behavior resulting in component scores with enough variability to distinguish between individuals. Different novel object tests elicited different responses. Of the four novel objects tested "ball" and "fruitcicle" elicited the widest variation between individuals and are, thus, our preferred measure of personality. Several direct behavioral measures obtained through novel object tests correlated significantly with appropriate keeper rating personality items. Theoretically, we would not expect specific time point samples of behavior during novel object tests to correlate perfectly with subjective keeper surveys. Instead, keeper questionnaires were designed to capture personality types that cannot be obtained through direct behavioral observations such as novel object tests or mirror-image stimulation (Wielebnowski 1999). Therefore, it is not surprising that some of the measurable behavioral events during novel object tests were not comparable to any of the keeper ratings. Our findings are consistent with other studies in cheetahs (Wielebnowski 1999), black rhinos (Carlstead et al. 1999a; Carlstead et al. 1999b), spotted hyenas (Gosling 2001), and preliminary studies on pandas (Powell \& Svoke 2008), which demonstrate the validity of subjective observer/keeper ratings. The current study also expanded on Powell \& Sovke's (2008) investigation in giant pandas that analyzed individualized behavioral reactions to novel objects and correlations with keeper surveys. As Powell and Svoke (2008) only sampled four animals across two institutions our study significantly increased samples sizes for more robust data analyses and addressed some of the questions unanswered in their study.

In this study, we found that animals labeled 'stereotypical' approached objects 
more quickly and had fewer chemical communication behaviors. Powell and Svoke (2008) found no significant correlations with stereotypical ratings and novel object behaviors. 'Vigilant' pandas also approached objects more quickly, had fewer chemical communication behaviors, and fewer aggressive vocalizations. The rating 'stereotypical' correlated highly with the rating 'vigilant' $(\mathrm{r}=0.47, p=0.005)$, thus, we propose that 'stereotypical' animals may be more 'vigilant' about new objects in their surroundings and this resulted in the high correlation of behavioral measurements across these two personality measurements. These questionnaire items seemed to correctly identify animals that were more aware of novel objects in their environment and adjusted their behaviors accordingly.

We also found that pandas that were labeled high on the 'aggressive' component (I) and 'aggressive towards conspecifics' by keepers approached novel objects slower and spent more time with the novel objects. We did not record whether novel object interactions appeared to be of an 'aggressive' nature or a 'curious' nature and suggest that future studies incorporate this information or include additional tests (i.e mirror simulation or unfamiliar human tests) that would capture this component better (Wielebnowski 1999). 'Aggressiveness' did not appear on any of Powell and Svoke (2008) correlations between novel object tests and personality traits. Similar to our study, Powell and Svoke (2008) research methods employed novel object tests and so, probably did not capture the aggressive component well. Ellis et al. (2006) found that 'aggression' was an important predictor of long-term breeding success for giant pandas and was negatively correlated with 'friendly to people', which is evident in factors loading on the 'aggressive' component (I) in our current study. Pandas that were 
'aggressive toward people' spent less time at the indoor enclosure door and had fewer chemical communication point samples. Powell and Svoke (2008) found pandas labeled "friendly toward people" visited the indoor enclosure door more often. Taken together and with Ellis et al.'s (2006) study, we suggest that 'friendly' pandas may actively seek human interaction by visiting enclosure doors whereas 'aggressive' pandas avoid human interaction.

'Excitable' pandas and pandas that were labeled as 'fearful' via component III also displayed fewer chemical communication behaviors. Because pandas that were 'fearful', 'excitable', 'stereotypical' and 'aggressive toward people' all had fewer chemical communication point samples, there appears to be a high correlation with lower chemical communication and animals that react negatively to typical captive conditions (i.e. interactions with people and sudden changes in the environment). Powell and Svoke (2008) found that scent marking was high in self-assured pandas. Taken together, we can surmise that rates of chemical communication may provide a reliable measure of animals that are comfortable in captivity versus those that react negatively to the captive environment. We suggest that future research investigates this relationship further.

\section{Reproductive performance and personality}

PCA analysis of questionnaire ratings obtained for giant pandas yielded four major components: 'aggressive', 'playful-clever', 'fearful', 'stereotypical-excitable'. These components appear to be equivalent to personality dimensions reported in other species such as humans (Cloninger et al. 1993), gorillas (Gold \& Maple 1994), spotted hyenas (Gosling 1998), cheetahs (Wielebnowski 1999), chimpanzees (Weiss et al. 2000), and bighorn sheep (Réale \& Festa-Bianchet 2003). We found that giant pandas that were 
most 'fearful' based on the PCA component were less likely to be natural breeders than animals that were not fearful. This result was confounded by the effect of age - older individuals were also more likely to be breeders. GLMMs revealed that age was the more important factor in determining both natural mating success and cub production when personality characteristics, natural history factors, and physiological factors were analyzed together. However, the 'aggressive' component was the second most important factor in predicting cub production with less aggressive females producing more cubs. As mentioned above, Ellis et al. (2006) found that 'aggression' was an important predictor of long-term breeding success, however, in contrast with our study, they found that highly aggressive giant pandas had higher long-term reproductive performance. Our methods of analyses were slightly different from Ellis et al. (2006) so it is hard to determine why our findings differed (see below for a more equivalent comparison). Future studies (or larger sample sizes) should control for the effect of age through analyzing animals within one standard deviation from the mean of the older age group in our study.

Animals that were labeled as 'fearful' were less likely to have natural mating than animals labeled low on this component. Surprisingly, and unlike Powell et al. (2008), we did not find any correlations with the 'shy' personality trait. 'Shy' loaded negatively on the 'aggressive' component but did not surface as a significant factor in our study. However, we believe that the component 'fearful' in our study is likely measuring similar personality traits. Definitions of 'shy' and 'fearful' could easily be misconstrued by keepers to mean the same thing (i.e. being "reluctant to engage in social situations" could be easily interchanged with "retreats and hides regularly from other pandas and people"). 
If we accept that these definitions may be interchangeable then our findings are in high agreement with Powell et al. (2008) in that shy/fearful individuals are less likely to have a natural mating that produces cubs and have lower total frequencies of mating behaviors. Boldness has been linked with increased fitness in both wild and captive studies and across species (Ariyomo et al. 2013; Réale \& Festa-Bianchet 2003; Smith \& Blumstein 2008).

Though personality types of male giant pandas did not correlate with cub production, female giant pandas that were less 'fearful' and less 'aggressive' in both keeper ratings, the 'aggressive' PCA component, and 'fearful' PCA component scores produced more cubs. The 'aggressive' effect on reproductive performance was opposite of what Ellis et al. (2006) reported where higher aggression scores increased reproductive performance in giant pandas. Our data suggests that high 'aggressiveness' in females is an important trait for natural mating success (Chapter 4) but there appears to be a trade off in females where the less 'aggressive' and less 'fearful' females produce more cubs. Though it may appear that Ellis et al.'s (2006) findings contradict ours, their study combined natural mating history, cub production, and litter survival into four general breeding categories whereas we analyzed natural mating history and cub production separately. Thus, Ellis et al. (2006) may have failed to capture differences between these phases through combining the three reproductive performance measurements.

Though 'fearful' and 'aggressive' component scores were not significantly different between captive-born and wild-born or between hand-reared and mother-reared individuals, the fact that these components showed trends with mother-reared animals being more 'playful' and wild-born individuals being more 'aggressive' raises some 
important concerns especially in light of our reproductive performance findings. If wild born animals are more 'aggressive' than captive, and more 'aggressive' animals are less likely to produce cubs, then we may be measuring the first signs of domestication effects in captivity. The argument could be made that having low 'aggressiveness' and 'fearfulness' may be a learned behavior in captivity. If these behaviors are learned and not under selection we would expect no difference between wild and captive-born individuals as the wild animals should learn to be less 'aggressive' and less 'fearful'. Theoretically, the captive environment may provide selection pressures that are different from the wild environment, which may manifest in differential reproductive performance in captivity (McDougall et al. 2006).

That females who were less 'fearful' and less 'aggressive' had higher cub production rates came as no surprise because these animals are probably less anxious than their 'fearful' and 'aggressive' counterparts. Females with lower 'fearful' and 'aggressive' personalities may have developed these personalities to cope with some of the stressors uniquely related to captivity. If fearful and aggressive animals are chronically stressed, this could explain their lower reproductive rates. Chronic states of anxiety have been found to correlate with reduced pre-ovulatory LH surge (Stoebel \& Moberg 1982), lower egg production in birds (Cyr \& Michael Romero 2007), lower gamete quality in fish (Campbell et al. 1994), smaller litter sizes and higher rate of fetal reabsorption in mice (Geber 1966), and can impair placental development and increase rates of spontaneous abortion in primates (Myers 1975; Small 1982). However, as suggested by (Powell et al. 2008) and Ellis et al. (2006) 'aggressiveness' seems to be a more complex characteristic than previously suspected. 


\section{Conclusions}

The results from this study suggest that behavioral personality assessment may allow us to predict an individual's ability to reproduce in captive environments. This study suggests that less 'fearful' individuals have higher reproductive performance in captivity and that this could be quantified at both the mating stage as well as in cub production. Individuals with high 'fearfulness' scores may have more difficulty coping with the captive environments than their less 'fearful' counterparts. Thus, extended captivity may change selection pressures such that less 'fearful' individuals have higher reproductive performance. Recent literature suggests that the repercussions of such an outcome may be detrimental to survivorship of reintroduced animals as bolder individuals usually have reduced survival due to increased risk-taking behaviors that frequently placed bold individuals in positions that would compromise survival (McPhee 2003, Smith 2008, McDougall et al. 2005). However, exactly how captivity is altering personalities across generations and the implications for reintroduction success has not been well studied and further investigation into the consequences of certain personality traits on fitness is needed.

Similar to McDougal et al. (2005) we suggest that future studies should concentrate on determining the trade-offs between increasing the breeding success for the entire captive population by selecting for breeding individuals with more appropriate temperaments for captivity and providing good breeding conditions for all individuals regardless of temperament. For example, when faced with a 'fearful' non-breeding female in captivity, breeding managers have the choice to either train the animal to become less 'fearful' or alter the breeding environment to accommodate for this 
'fearfulness' through decreasing human presence or offering a more naturalistic breeding enclosure. Until relationships between fitness and personality can be more fully determined, we would caution against suggestions that managers should take efforts to reduce fearfulness in certain individuals to increase reproductive performance in captivity as (depending on whether this behavior is learned or genetic) this may lead to a reduction in variation in personality traits and select for traits that may be maladaptive for reintroduced animals (e.g. 'fearfulness' may be necessary for cub survivorship when giant pandas are susceptible to predators; McDougall et al. 2005, Smith et al. 2008). However, altering husbandry practices to accommodate for a wide variety of personality traits (i.e. offering fearful animals quieter and less distracting enclosures for breeding opportunities) could increase reproductive performance in the captive population while simultaneously maintaining both the genetic and behavioral diversity captive programs strive to obtain.

\section{Acknowledgments}

We thank the CCRCGP giant panda keepers, researchers and staff for all their help and support. This work was supported by an Animal Behavior Society Student Research Grant, an Oregon Zoo Future for Wildlife Grant, and a Portland State University's Forbes Lea Grant. We thank our hard working interns, Arina Woolery, Kim Render, Stephanie McMahon, Amelie LeRoy, and Grace Russell - without their hard work none of this research would have been accomplished. 
Table 3.1. Behavioral definitions of adjectives used for questionnaire ratings. Definitions are obtained or modified from Powell et al. (2008), Wielebnowski (1999), and Shepherdson et al. (2004).

\begin{tabular}{|c|c|}
\hline Vigilant & Pays attention to the surroundings and changes in surroundings \\
\hline Active & Moves frequently (e.g. walks, climbs, paces a lot) \\
\hline Aggressive to conspecifics & Frequently reacts hostile (e.g. attacks, growls) toward other pandas \\
\hline Aggressive to people & Frequently reacts hostile and threatening toward people \\
\hline Calm & Not easily disturbed by changes in the environment \\
\hline Curious & Readily approaches and explores changes in the environment \\
\hline Stereotypical & Shows stereotypic or unusual behaviors \\
\hline Excitable & Overreacts to changes in the environment \\
\hline Friendly to conspecifics & Social; initiates and seems to seek proximity of other pandas \\
\hline Friendly to people & $\begin{array}{l}\text { Initiates proximity; approaches enclosure bars readily and in a } \\
\text { friendly manner }\end{array}$ \\
\hline Fearful of conspecifics & Retreats and hides readily from other pandas \\
\hline Fearful of people & Retreats readily from people \\
\hline Anxious & Seems uneasy and worried about the environment or new events \\
\hline Playful & $\begin{array}{l}\text { Initiates and engages in play behavior (seemingly meaningless, but } \\
\text { non-aggressive behavior) with objects and/or other pandas }\end{array}$ \\
\hline Self-confident & $\begin{array}{l}\text { Moves in a seemingly confident, well -coordinated, and relaxed } \\
\text { manner }\end{array}$ \\
\hline Clever & $\begin{array}{l}\text { Learns quickly to associate certain events and appears to remember } \\
\text { for a long time. }\end{array}$ \\
\hline Innovative & Seems original and creative; solves problems \\
\hline Solitary & Spends time alone; avoids company \\
\hline Nervous & Shows restraint in movement and posture; easily agitated or alarmed \\
\hline Vocal & Frequently and readily vocalizes \\
\hline Bad tempered & Easily annoyed or made angry \\
\hline Not Interested & $\begin{array}{l}\text { Unresponsive to and seemingly unaware of significant } \\
\text { events/situations }\end{array}$ \\
\hline Shy & Reluctant to engage in social situations \\
\hline
\end{tabular}


Table 3.2. Behavioral definitions of adjectives used for questionnaire ratings. Behaviors are obtained or modified from Powell and Svoke (2008)

Object Interaction

Latency to touch

Total time

Total visits

Total point samples

Door directed behaviors

Total time

Total point samples

Stereotypical behaviors

Total time

Total different types

Total point samples

Total active behaviors

Total Chemical Communication

Total Vocal Communication Affiliative

Aggressive
Any behaviors that involved direct interaction with the object such as manipulating, pawing, biting, carrying, and pushing

Behavior and attention is directed toward the inner enclosure door toward food, keeper, or bedroom area and away from the object.

Animal engages in unvarying and repetitive (three times or more) acts that have no obvious goal or function.

Any behaviors that involve movement of bear including sustained locomotion in a non-stereotyped manner, searching for food, climbing, and responding to stimuli.

Any behaviors that involve scent marking, body rubbing, scent anointing, sniffing/olfactory investigation, flehmen, urinating, and defecating.

Affiliative vocalizations include: bleats, chirps and sex squeal.

Aggressive vocalizations include: chomps, huffs, moans, snorts, barks, growls, and roars. 
Table 3.3. Four major components of individual behavior variation in captive giant pandas derived from combined score results of keeper questionnaires on 33 adult giant pandas at the China Conservation and Research Center for Giant Pandas obtained through principal component analysis. Components accounted for $53 \%$ of the variance seen in individual pandas.

Component

\begin{tabular}{lrrrr} 
Variable & I & \multicolumn{1}{c}{ II } & III & \multicolumn{1}{c}{ IV } \\
\hline Vigilant & 0.10 & -0.08 & -0.08 & $0.70^{\mathrm{a}}$ \\
Active & -0.12 & 0.34 & -0.29 & 0.27 \\
Aggressive to conspecifics & $0.82^{\mathrm{a}}$ & -0.20 & -0.27 & -0.03 \\
Aggressive to people & $0.82^{\mathrm{a}}$ & -0.06 & 0.12 & 0.13 \\
Calm & -0.20 & -0.07 & 0.03 & 0.08 \\
Curious & 0.08 & 0.08 & -0.44 & 0.35 \\
Eccentric & 0.02 & -0.03 & -0.07 & $0.84^{\mathrm{a}}$ \\
Stereotypical & 0.15 & 0.04 & 0.10 & $0.85^{\mathrm{a}}$ \\
Friendly to conspecifics & -0.78 & -0.02 & -0.20 & -0.21 \\
Friendly to people & -0.48 & 0.29 & -0.53 & 0.00 \\
Fearful of conspecifics & -0.16 & -0.10 & $0.85^{\mathrm{a}}$ & -0.09 \\
Fearful of people & 0.07 & 0.13 & $0.81^{\mathrm{a}}$ & 0.04 \\
Anxious & 0.44 & 0.05 & 0.20 & 0.33 \\
Playful & -0.22 & $0.83^{\mathrm{a}}$ & -0.9 & 0.10 \\
Self-confident & 0.10 & $0.56^{\mathrm{a}}$ & -0.64 & -0.10 \\
Clever & 0.25 & $0.79^{\mathrm{a}}$ & -0.27 & -0.01 \\
Innovative & 0.21 & $0.74^{\mathrm{a}}$ & 0.05 & -0.28 \\
Solitary & $0.50^{\mathrm{a}}$ & -0.58 & 0.04 & -0.10 \\
Nervous & 0.00 & -0.40 & $0.50^{\mathrm{a}}$ & $0.50^{\mathrm{a}}$ \\
Vocal & -0.03 & 0.05 & -0.23 & 0.01 \\
Bad tempered & 0.36 & -0.03 & 0.43 & 0.38 \\
Not Interested & 0.08 & -0.33 & $0.73^{\mathrm{a}}$ & -0.11 \\
Shy & -0.46 & -0.39 & -0.03 & 0.09 \\
\hline
\end{tabular}

${ }^{\mathrm{a}}$ Component loadings $\geq 0.5$ 
Table 3.4. Five major components of individual behavior variation in captive giant pandas derived from behavioral scores on ball novel object test administered to adult giant pandas at the China Conservation and Research Center for Giant Pandas obtained through principal component analysis.

Component

\begin{tabular}{lccccc} 
Variable & I & II & III & IV & V \\
\hline Latency to Touch (sec) & 0.00 & -0.10 & -0.14 & -0.05 & $0.94^{\mathrm{a}}$ \\
Total time with novel object (min) & 0.43 & 0.02 & $0.84^{\mathrm{a}}$ & -0.13 & -0.03 \\
Total number of object interactions & 0.19 & 0.27 & $0.89^{\mathrm{a}}$ & 0.06 & -0.09 \\
Total time at shift door (min) & -0.01 & $0.95^{\mathrm{a}}$ & 0.16 & 0.02 & -0.06 \\
Number of visits to shift door & 0.05 & $0.98^{\mathrm{a}}$ & 0.03 & 0.06 & -0.06 \\
Point samples & & & & -0.00 \\
$\quad$ Total active & $0.93^{\mathrm{a}}$ & -0.02 & 0.28 & 0.01 & 0.01 \\
$\quad$ Total chemical communication & 0.18 & 0.15 & -0.20 & $0.81^{\mathrm{a}}$ & -0.10 \\
$\quad$ Total stereotypical behaviors & $0.54^{\mathrm{a}}$ & 0.32 & -0.46 & 0.15 & 0.17 \\
Total number of scent marks & -0.13 & -0.05 & 0.09 & $0.90^{\mathrm{a}}$ & -0.02 \\
Total time in stereotypical behavior & $0.92^{\mathrm{a}}$ & -0.02 & 0.26 & -0.02 & $0.80^{\mathrm{a}}$ \\
Total negative vocalizations & -0.07 & -0.03 & 0.10 & 0.48 & -0.03 \\
Total affiliative vocalizations & 0.04 & 0.06 & -0.12 & -0.09 & -0.09 \\
\hline
\end{tabular}

${ }^{\mathrm{a}}$ Component loadings $\geq 0.5$ 


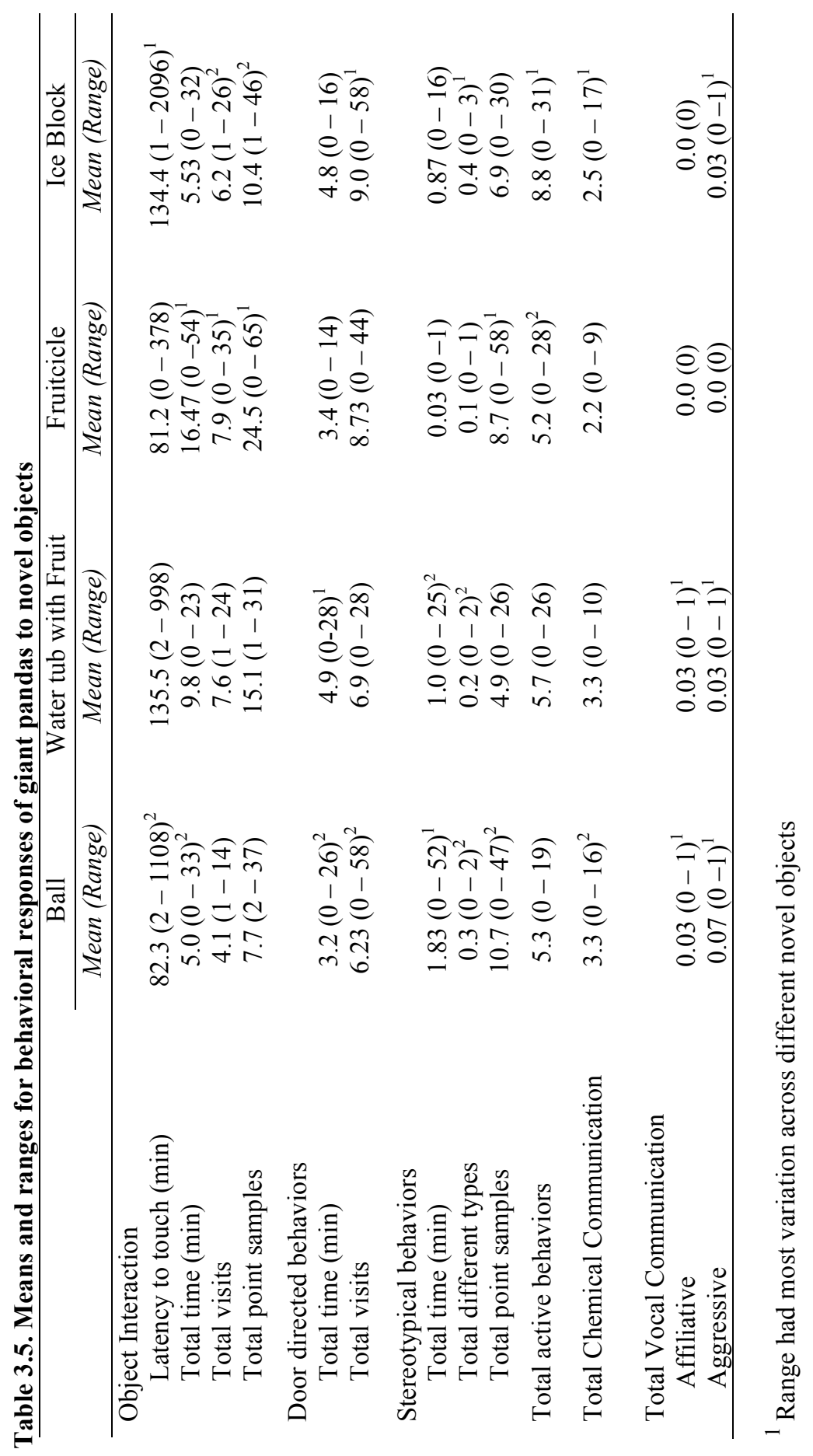




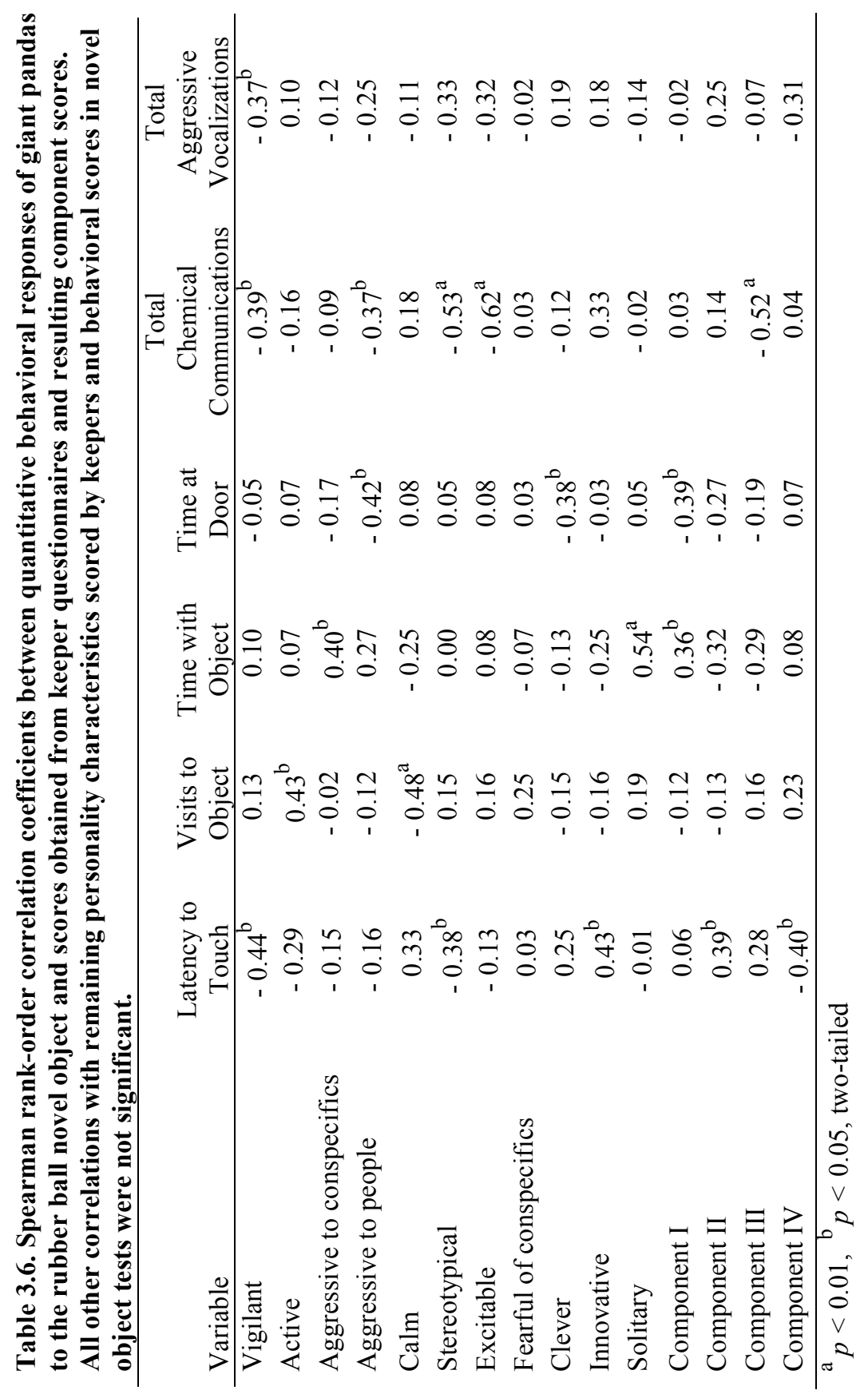




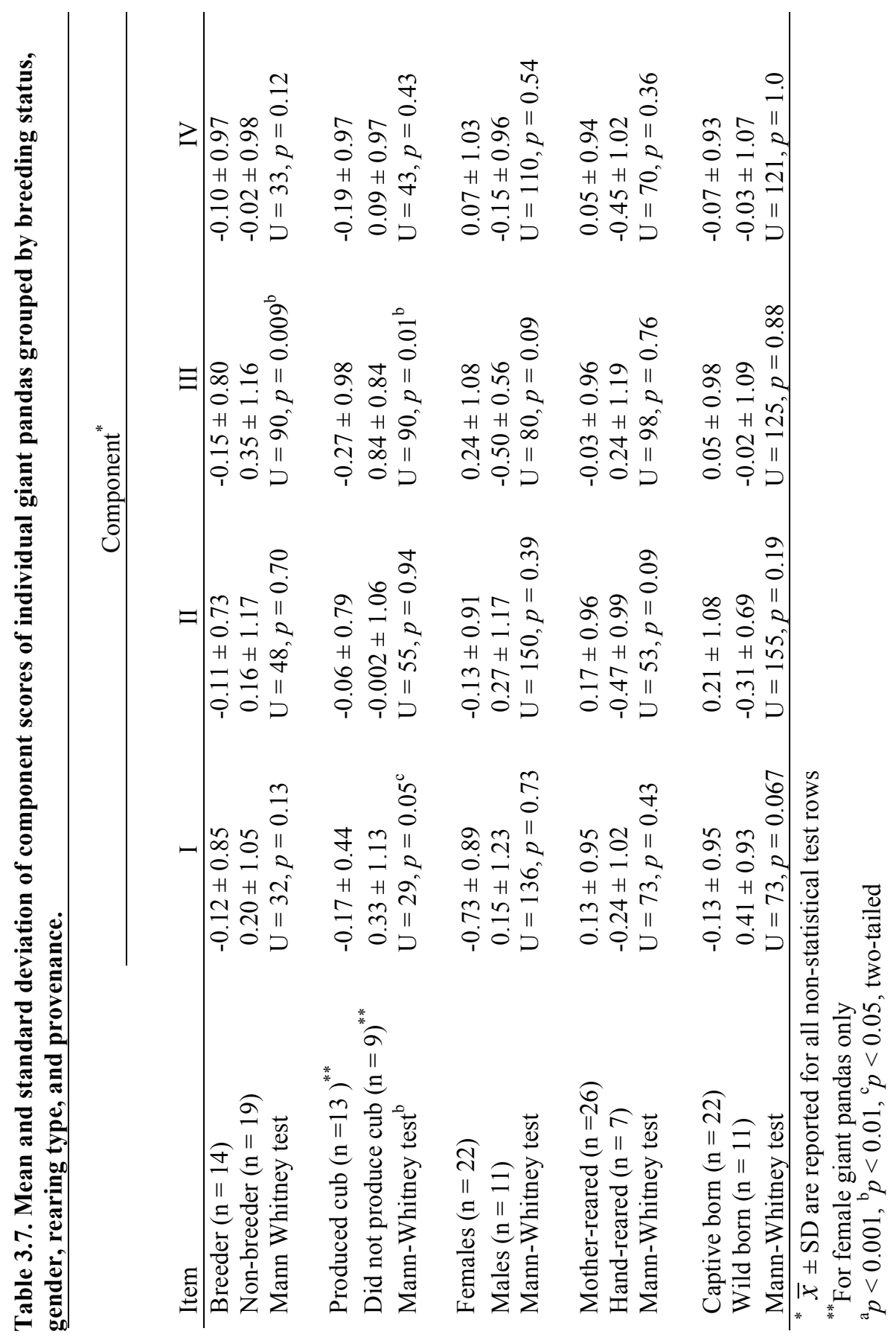


Table 3.8. Spearman rank-order correlation coefficients between average mating behaviors of giant pandas to scores obtained from keeper questionnaires. All other correlations with remaining personality characteristics scored by keepers and obtained from PCA component scores were not significant.

\begin{tabular}{|c|c|c|c|}
\hline \multirow[b]{2}{*}{ Variable } & \multicolumn{3}{|c|}{ Mating Behaviors } \\
\hline & $\begin{array}{l}\text { Average } \\
\text { Frequency } \\
\text { Positive }\end{array}$ & $\begin{array}{c}\text { Average } \\
\text { Frequency } \\
\text { Negative }\end{array}$ & $\begin{array}{c}\text { Average } \\
\text { Frequency } \\
\text { Total } \\
\end{array}$ \\
\hline \multicolumn{4}{|l|}{ Both Sexes Combined } \\
\hline Vigilant & 0.04 & -0.16 & $0.45^{\mathrm{b}}$ \\
\hline Curious & -0.36 & $0.38^{\mathrm{c}}$ & 0.09 \\
\hline Friendly toward people & $-0.39^{\mathrm{b}}$ & $0.38^{\mathrm{c}}$ & -0.19 \\
\hline Fearful of conspecifics & -0.28 & 0.08 & $-0.76^{\mathrm{a}}$ \\
\hline Fearful of people & 0.14 & $-0.45^{\mathrm{b}}$ & $-0.36^{\mathrm{c}}$ \\
\hline Vocal & -0.36 & -0.01 & -0.09 \\
\hline Age & -0.12 & -0.22 & -0.09 \\
\hline Mass & 0.26 & $0.47^{\mathrm{b}}$ & $0.52^{\mathrm{b}}$ \\
\hline \multicolumn{4}{|l|}{ Females Only } \\
\hline Friendly toward people & -0.26 & $0.60^{\mathrm{b}}$ & $0.46^{\mathrm{c}}$ \\
\hline Curious & -0.11 & $0.46^{\mathrm{b}}$ & 0.35 \\
\hline Fearful of conspecifics & $-0.68^{\mathrm{a}}$ & 0.09 & -0.07 \\
\hline Component I & $0.58^{\mathrm{b}}$ & -0.16 & 0.14 \\
\hline Age & -0.22 & -0.26 & -0.20 \\
\hline Mass & -0.06 & $0.48^{\mathrm{c}}$ & 0.40 \\
\hline \multicolumn{4}{|l|}{ Males Only } \\
\hline Fearful of conspecifics & $-0.91^{\mathrm{a}}$ & 0.03 & $-0.91^{\mathrm{a}}$ \\
\hline Innovative & $-0.73^{c}$ & 0.10 & $-0.69^{c}$ \\
\hline Component II & $-0.65^{\mathrm{c}}$ & 0.57 & -0.55 \\
\hline Component III & -0.58 & 0.05 & $-0.67^{\mathrm{c}}$ \\
\hline Age & 0.26 & 0.31 & 0.27 \\
\hline Mass & 0.57 & $0.66^{\mathrm{c}}$ & $0.71^{\mathrm{c}}$ \\
\hline
\end{tabular}

${ }^{\mathrm{a}} p<0.001,{ }^{\mathrm{b}} p<0.01,{ }^{\mathrm{c}} p<0.05$, two-tailed 


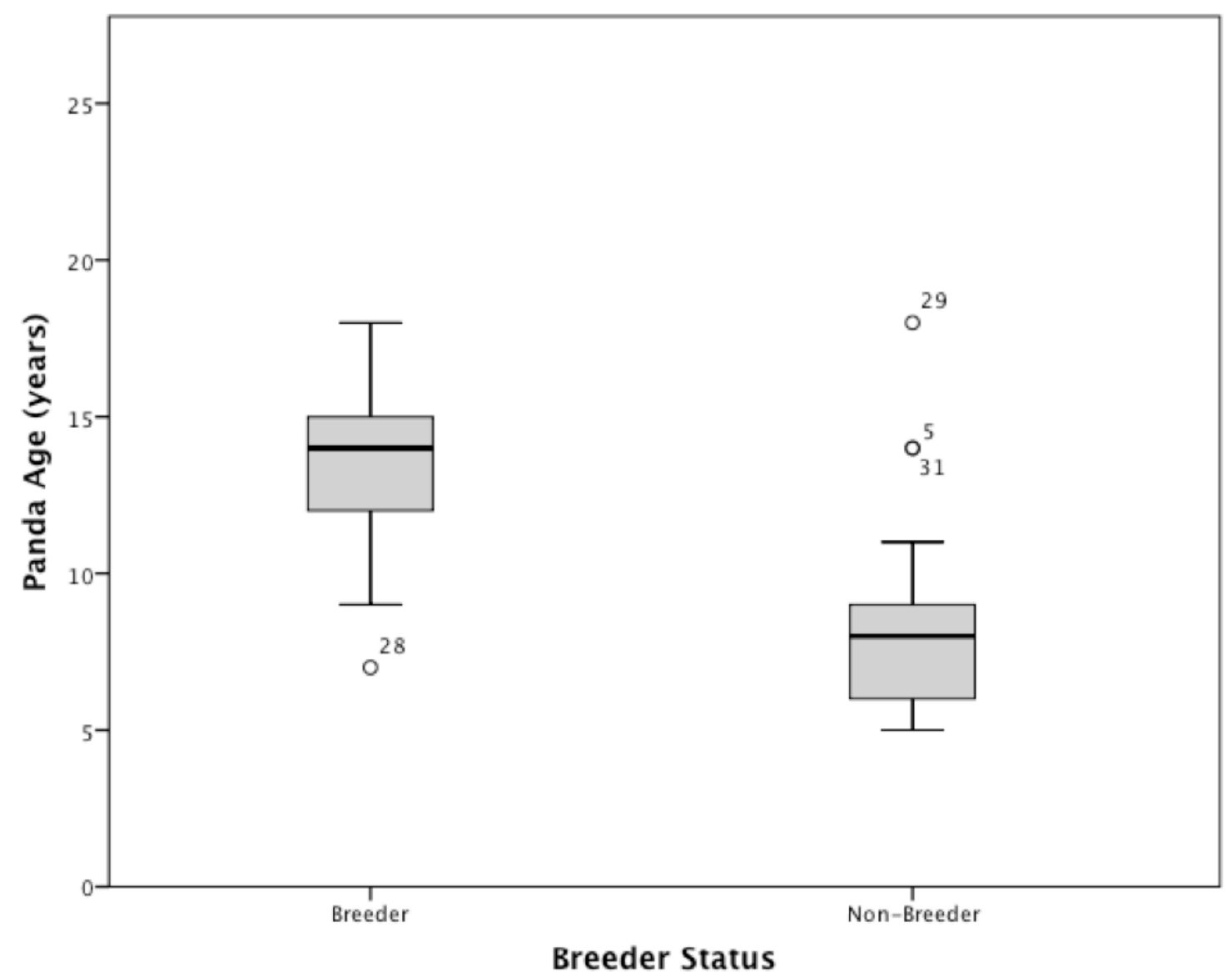

Figure 3.1. Mean age of breeder and non-breeder giant pandas at the China Conservation and Research Center for the Giant Panda in 2012 and 2013. 


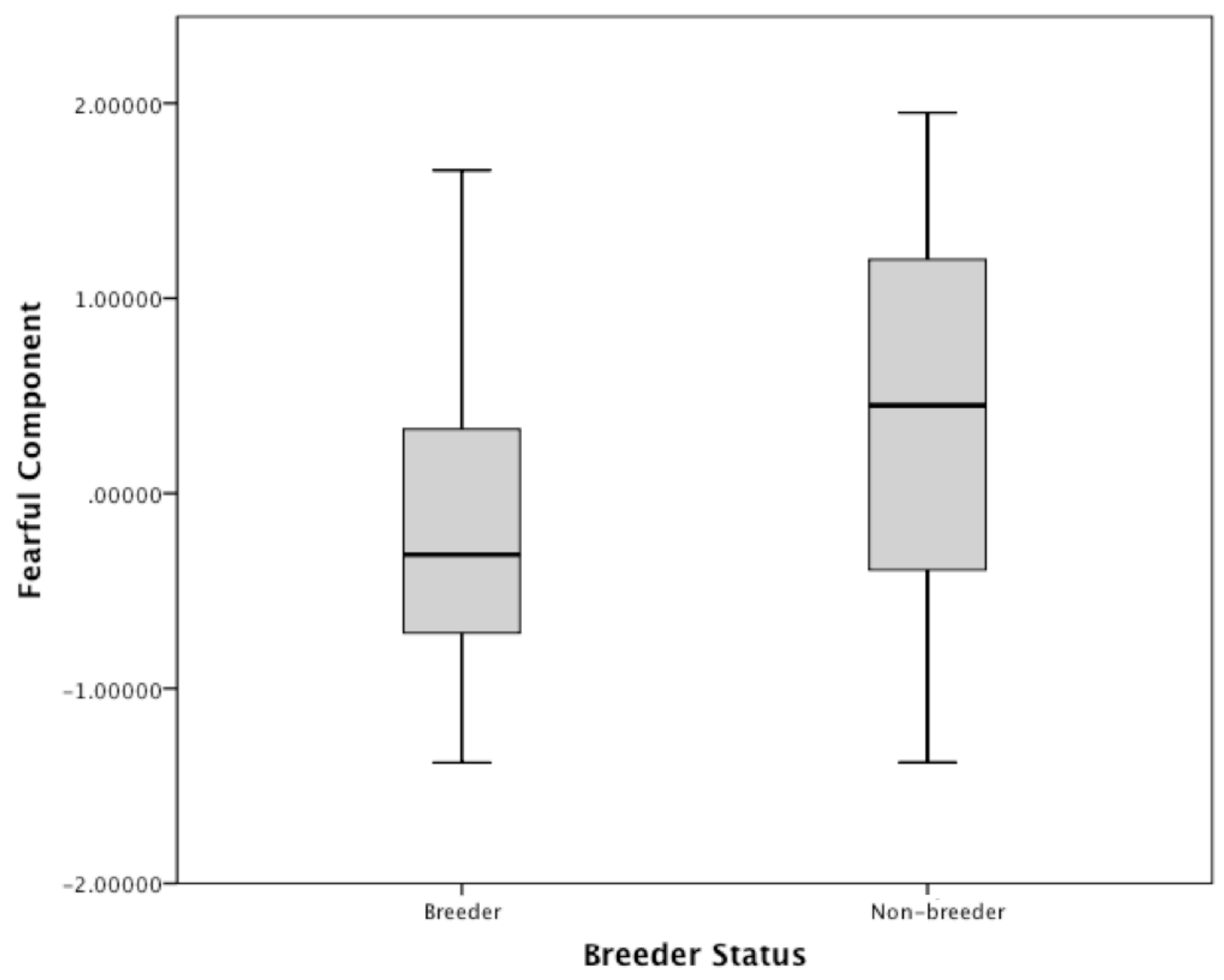

Figure 3.2. Mean fearful component scores of breeder and non-breeder giant pandas at the China Conservation and Research Center for the Giant Panda in 2012 and 2013. 


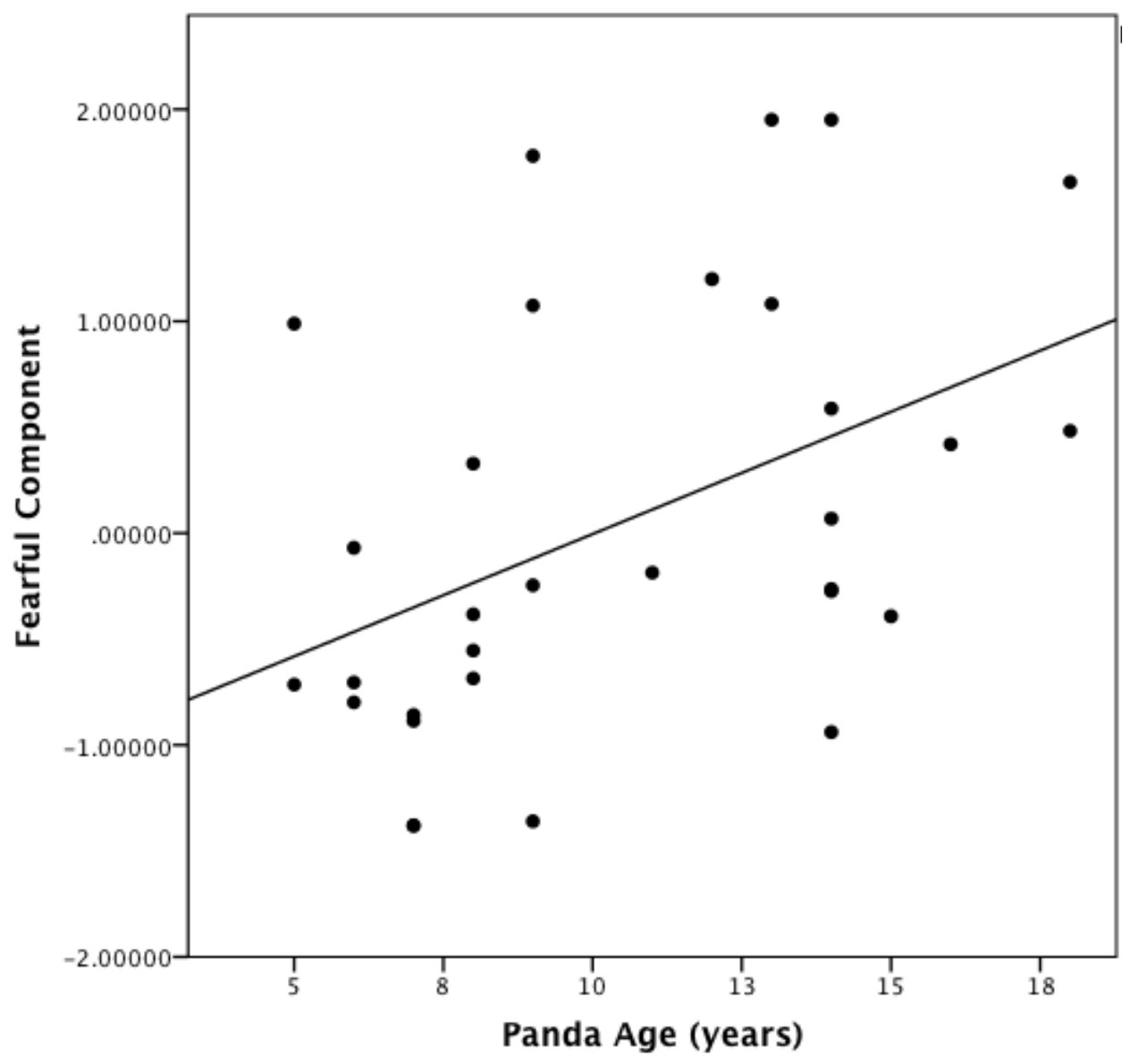

Figure 3.3. Fearful Component as a function of age in giant pandas at the China Conservation and Research Center for the Giant Panda in 2012 and 2013. 


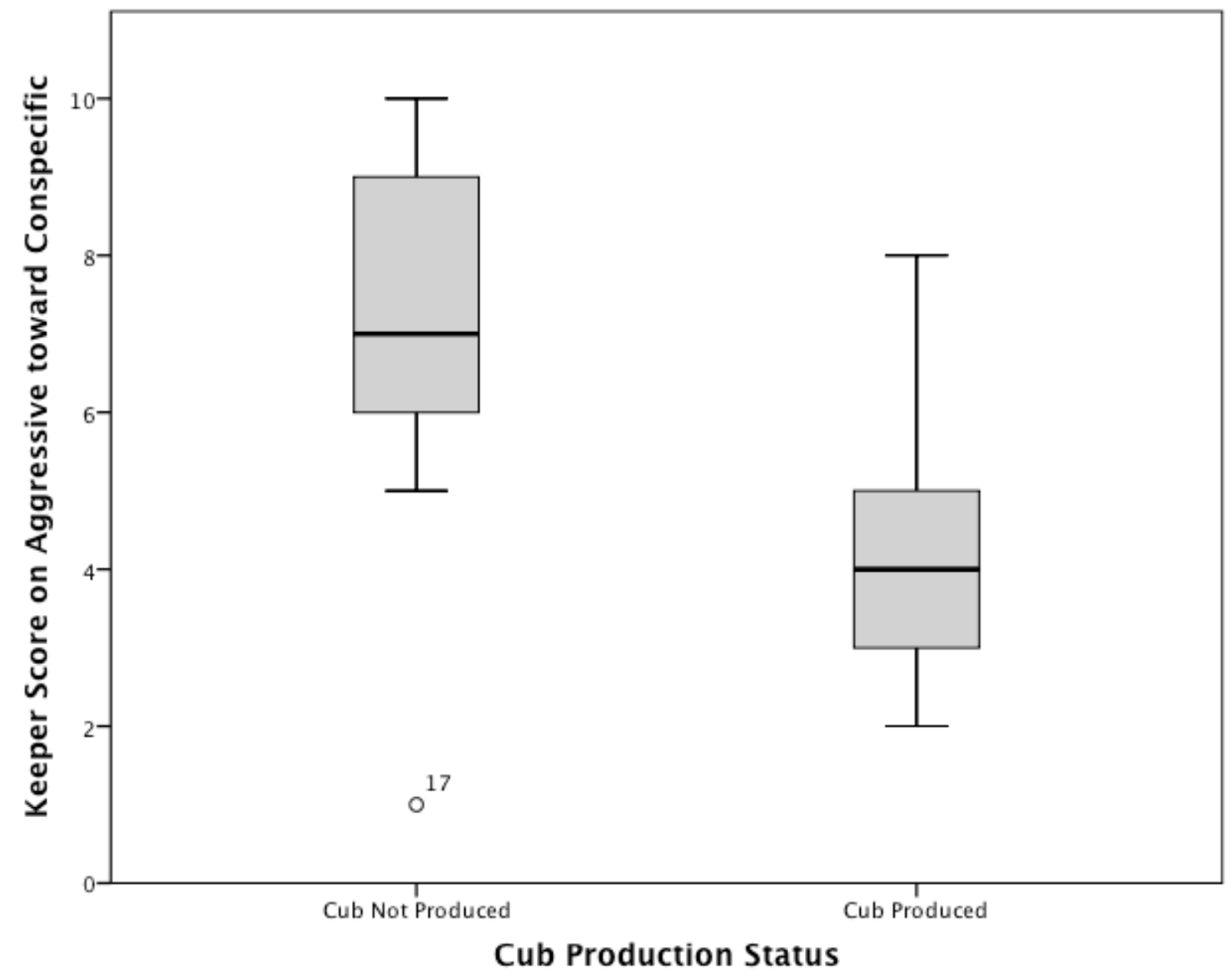

Figure 3.4a. Average keeper scores on 'aggressive toward conspecifics' questionnaire factor for female giant pandas that produced cubs and did not produce cubs. 


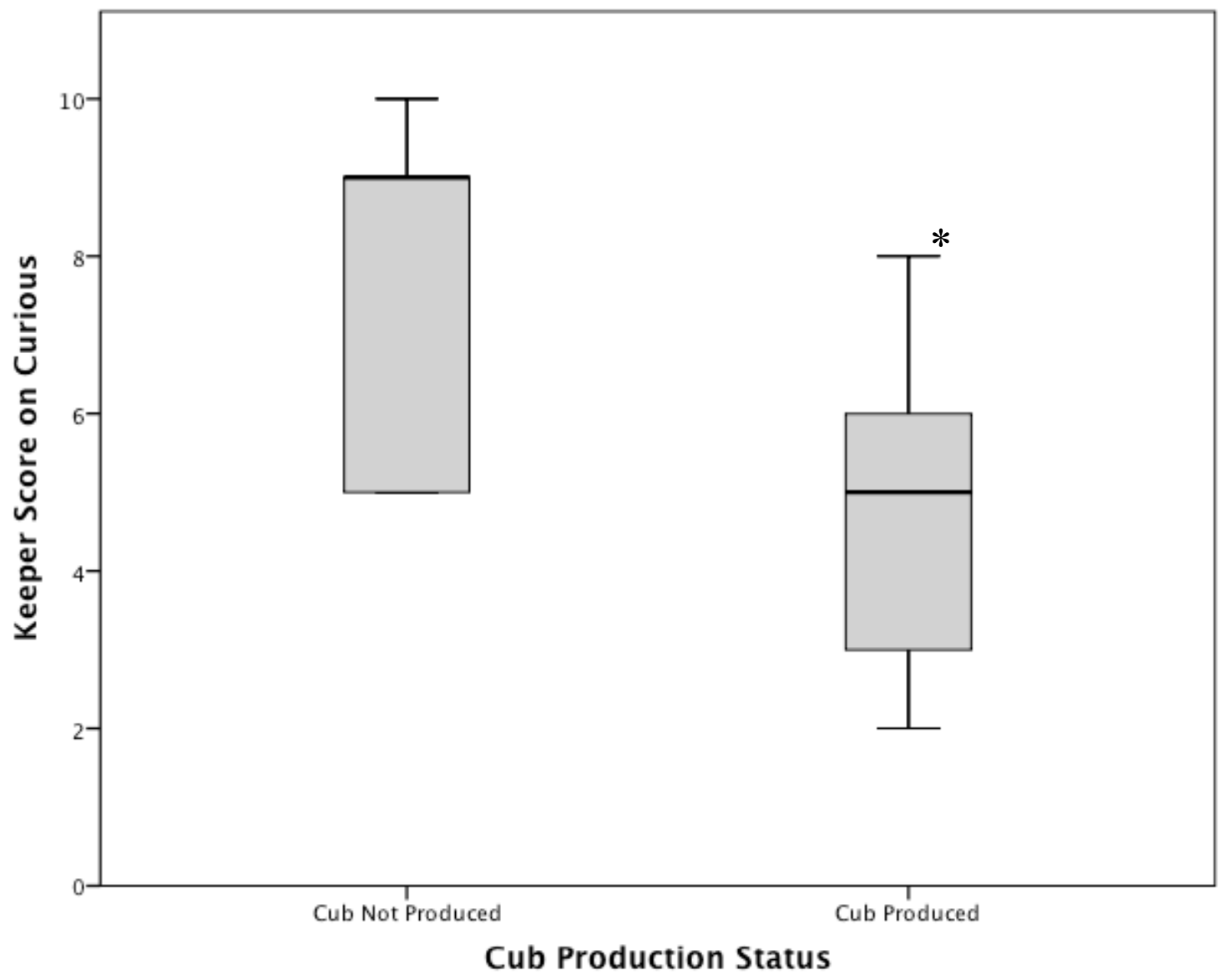

Figure 3.4b. Average keeper scores on 'curious' questionnaire factor for female giant pandas that produced cubs and did not produce cubs. 


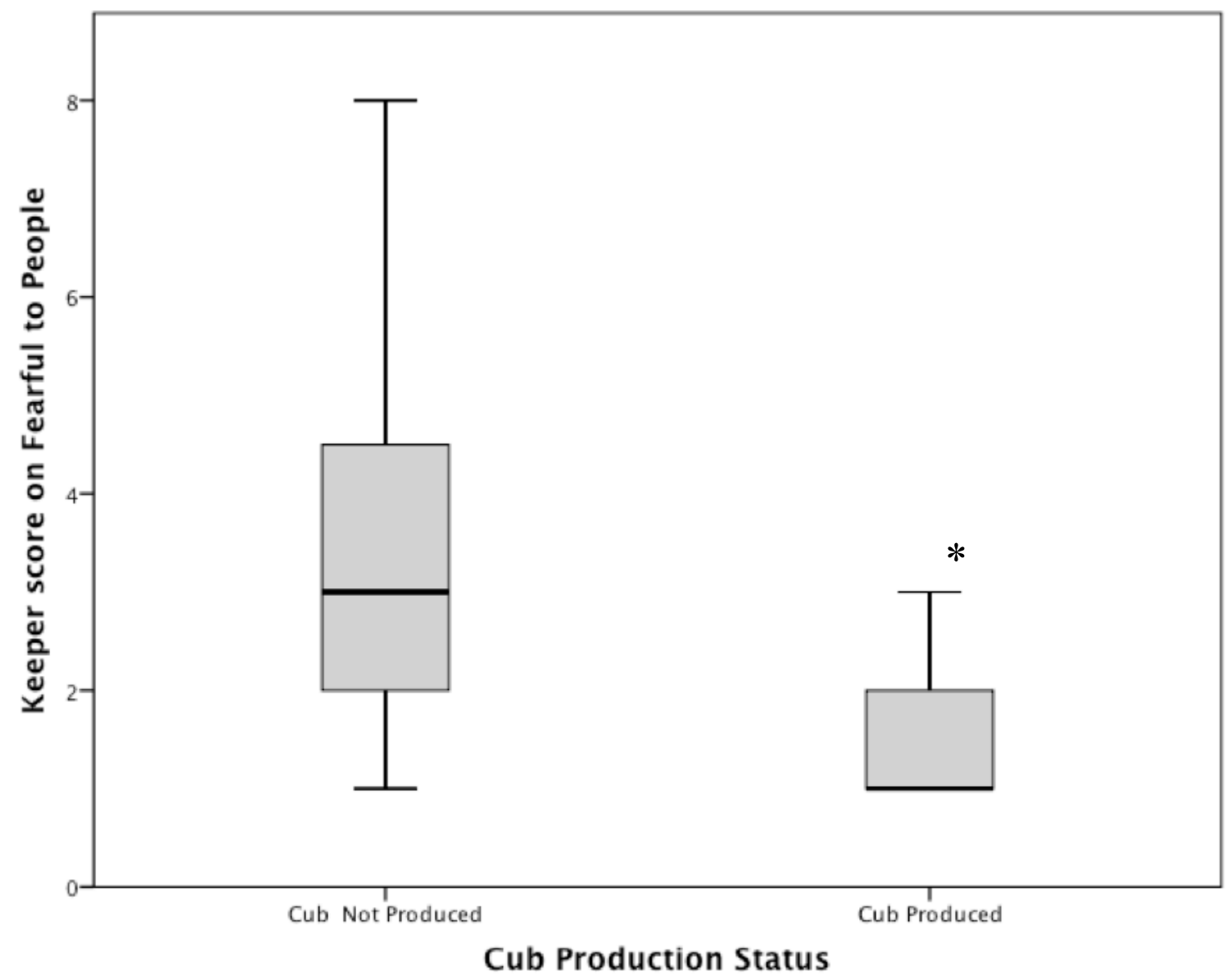

Figure 3.4c. Average keeper scores on 'fearful of people' questionnaire factor for female giant pandas that produced cubs and did not produce cubs. 


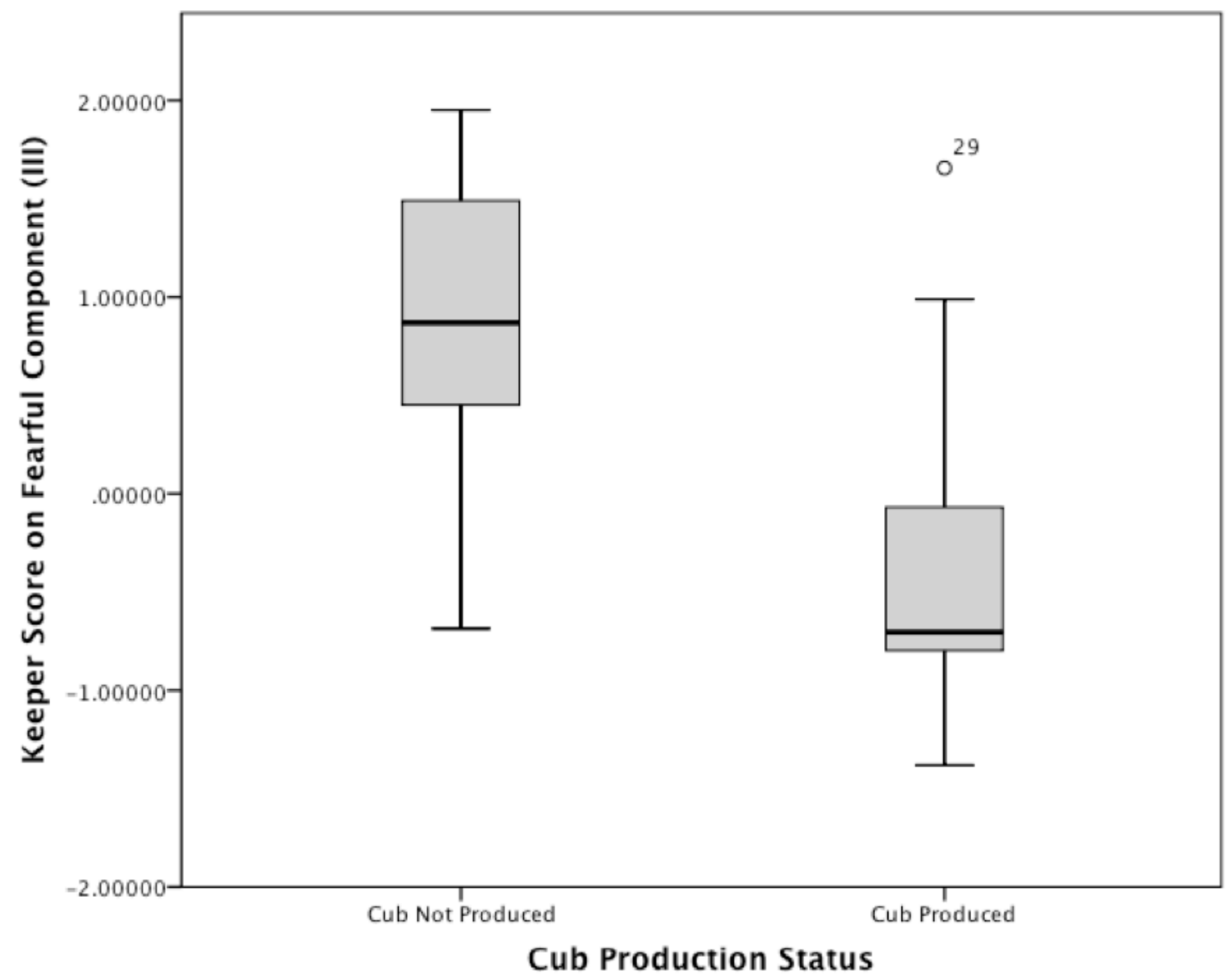

Figure 3.4d. Average scores on fearful component (III) for female giant pandas that produced cubs and did not produce cubs. 


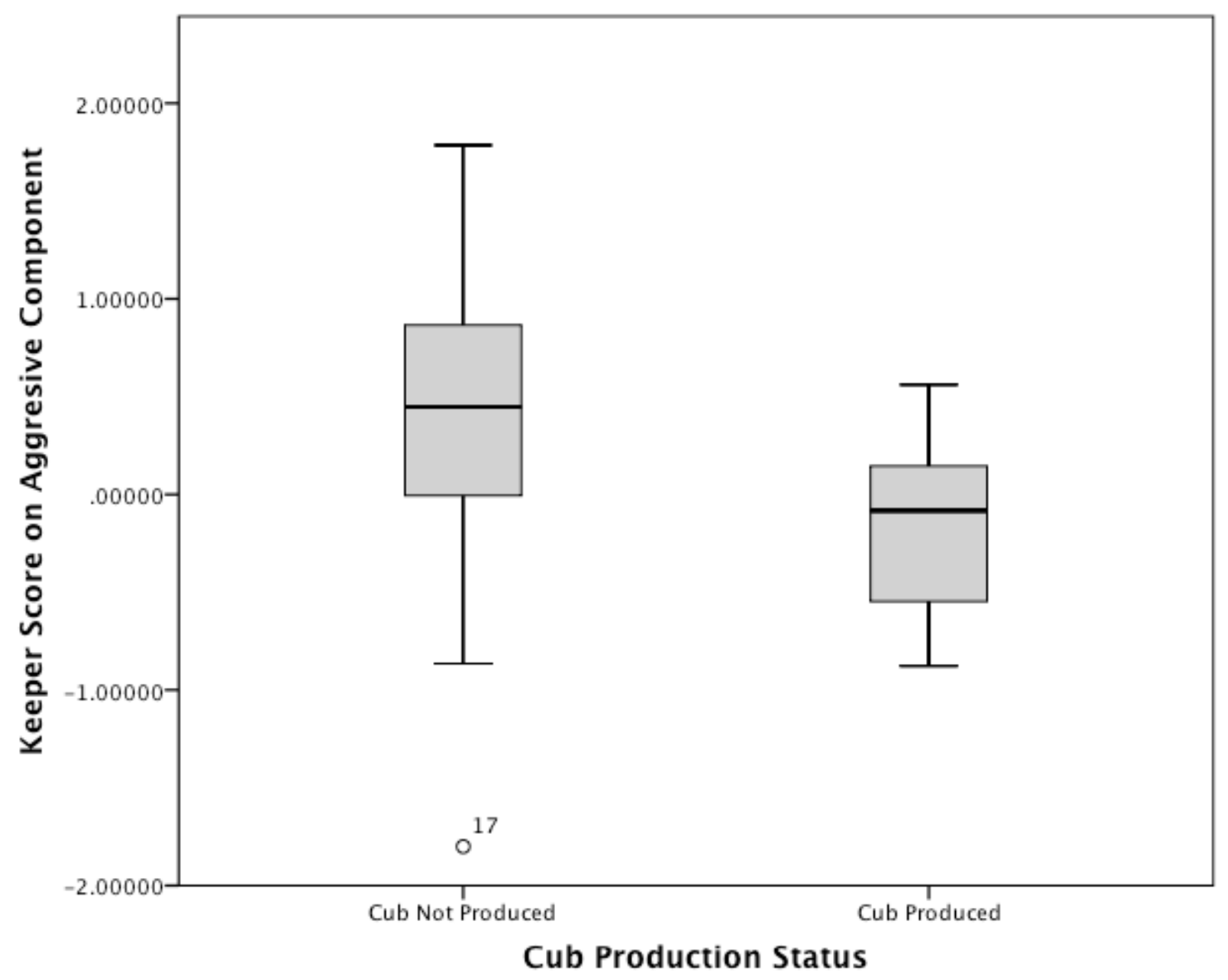

Figure 3.4e. Average scores on aggressive component (I) for female giant pandas that produced cubs and did not produce cubs. 


\section{CHAPTER 4: DO OPPOSITES ATTRACT? PERSONALITY DISSIMILARITY \\ IN BREEDING PAIRS OF CAPTIVE GIANT PANDAS INCREASE REPRODUCTIVE PERFORMANCE}

Running head: Mate pair personality dissimilarity and reproductive performance in giant pandas

Keywords: personality, temperament, reproductive performance, captive breeding, giant panda, mate compatibility, mate matching

Word Count: 6445

Page Count: 24 


\begin{abstract}
Recent studies, mainly in birds, suggest that mate compatibility within personality types can improve reproductive performance measurements such as mating success, offspring survivorship, and offspring quality. We investigated the role of personality matching on reproductive performance in male and female giant pandas as measured by intromission success and litter production. We conducted these studies on giant pandas at the China Conservation and Research Center for the Giant Panda (Bifengxia, Sichuan, China.) from February-May in 2011, 2012, and 2013. We used keeper surveys to personality-type male and female giant pandas on four major personality characteristics obtained from principal component analysis from our previous study; aggressive, playfulclever, fearful, and stereotypical-excitable (Chapter 3). Here we further investigate the effect of personality compatibility on reproductive performance by analyzing mate dyad similarity (similar versus dissimilar), specific personality combinations, and relative personality scores (male scores higher or lower than female scores). The specific personality combinations were; females and males that were both low on the personality component (L_L), females were low but males were high $\left(\mathrm{L}_{-} \mathrm{H}\right)$, females were high but males were low $\left(\mathrm{H} \_\right.$L), and females and males were both high $\left(\mathrm{H}_{-} \mathrm{H}\right)$. Our results suggest that giant pandas appear to be assortatively mating based on aggressive, fearful, and stereotypical-excitable personality traits. Mate dyads that were dissimilar on the stereotypical-excitable component score were more likely to have successful intromission than pairs that were similar. Further analyses indicated that specific combinations of personalities conferred increased reproductive performance measurements. Mate dyads composed of females ranked low and males ranked high ( $\mathrm{L}_{-} \mathrm{H}$ mate dyads) on both the
\end{abstract}


aggressive and stereotypical-excitable personality traits had increased intromission success and cub production than all possible personality combinations. In addition, mate dyads that were composed of males and females both ranked 'low' $\left(L_{-} L\right)$ on fearfulness had higher intromission success but not higher cub production than all other possible personality combinations. Mate dyads composed of males ranked high on the aggressive component relative to the female resulted in higher reproductive performance and cub production. No other relative personality-types conferred reproductive benefits to a mating pair. Our results suggest that integrating genetic information with behavioral measures of personality may increase the reproductive output of the giant panda captivebreeding program. 


\section{Introduction}

Conservation breeding to restore and/or supplement wild populations is an increasingly important tool for the recovery of threatened and endangered species. However, efforts to create self-sustaining populations of endangered species through captive breeding have been largely disappointing. Lees and Wilcken (2009b) analyzed 87 mammal populations in zoos and found that $52 \%$ were not breeding to replacement and that $67 \%$ fell below the threshold of 200 animals recommended to sustain genetically healthy populations by Baker (2007). In captive-breeding programs, breeding pairs are typically chosen on the basis of minimizing inbreeding and maintaining founder representation. Though these goals are widely recognized as important, they have emphasized genetic diversity sometimes at the expense of behavioral diversity (Rabin 2003; Swaisgood \& Schulte 2010). Such breeding techniques rely heavily on behavioral compatibility of the genetically recommended male and females. If a pair is behaviorally incompatible and refuses to mate than the unique genetic contribution of that pair is entirely lost to the captive population. Thus, improving behavioral mate compatibility to increase reproductive performance is particularly important in endangered species captive breeding where program success may mean the difference between survival of a species or extinction.

Recent research suggests that mate compatibility between partners in both physiological (Choudhury et al. 1996; Marzluff et al. 1996) and behavioral measurements (Both et al. 2005; Dingemanse et al. 2004; Dingemanse \& Réale 2005; Gabriel \& Black 2012), may confer a fitness benefit to compatible pairs versus non-compatible pairs. Of the few studies that exist which assess the link between personality and sexual selection, 
evidence exists for negative- and positive-assortative mating for certain personality combinations (typically similar personalities; reviewed in Schuett et al. 2010) and increased reproductive performance, offspring quality, and offspring survivorship (Both et al. 2005; Budaev 1999; Gabriel \& Black 2012; Sinn et al. 2006). For example, intermediate and bold dumpling squid, Euprymna tasmanica, are more likely to reproduce if they have behaviorally similar mates (Sinn et al. 2006). Similarly, convict cichlid pairs that reproduce show more similar behavioral types to one another than nonreproducing pairs (Budaev 1999). Mate pairs of great tits that display similar exploratory behaviors, Parus major, raise chicks in better condition (Both et al. 2005) and recruit more offspring than dissimilar pairs (Dingemanse et al. 2004). Finally, Stellar jay mate pairs composed of males and females that are similar in 'explorative tendencies' and in 'willingness to take risks' initiated nests earlier and were more likely to fledge offspring than dissimilar pairs (Gabriel \& Black 2012). Mates may choose partners with specific personality traits because of genetic compatibility, because some personality characteristics indicate quality, or certain personality types might be able to provide more effective parental care (Schuett et al. 2011). Taken together, these findings suggest that animals may non-randomly choose mates based on personality and these choices may have reproductive consequences.

This applies as well for captive breeding and recovery programs of endangered mammalian species. Personality characteristics have also been associated with increased reproductive performance for captive management and breeding of mammals in zoos and breeding facilities (Carlstead et al. 1999a; Carlstead et al. 1999b; Gold \& Maple 1994; Mendl et al. 1992; Powell \& Svoke 2008). For example, male black rhinoceros that are 
scored as being more dominant to conspecifics by their keepers have lower reproductive performance (Carlstead et al. 1999b) and nonbreeding captive cheetahs were rated by their keepers as significantly more tense-fearful than breeding cheetahs (Wielebnowski 1999). McDougall et al. (2006) believe that breeding programs that ignore temperament risk leading the population towards domestication because certain personalities may be more likely to prosper in captive settings. Therefore, the knowledge gained from personality studies can be useful in optimizing captive reproduction and increasing reintroduction success (McDougall et al. 2006). However, studies investigating the role of personality matching between mating pairs have not been conducted in captive endangered species despite the clear utility of such an approach.

Thus, in the present study, we investigated giant panda personalities obtained from keeper surveys to evaluate whether pairs with similar personality characteristics had higher reproductive performance than pairs that were dissimilar. We have recently shown that less fearful giant pandas have higher long-term reproductive performance, as measured by natural intromission success and cub production, than their more fearful counterparts (Chapter 3). Also that aggressive female giant pandas were more likely to have natural mating success but not more likely to produce cubs than nonaggressive females (Chapter 3). A previous study by Powell and Svoke (2008) also showed that shyer female pandas were more likely to be aggressive during mating and were less likely to display interest in the males with which they were paired. In addition, Ellis et al. (2006) showed that high aggression in giant pandas correlated with being a "prime breeder' as measured by grouping long-term natural mating success, cub production, and offspring survivorship. However, none of these studies looked at the relative personality 
of a mate pair and subsequent reproductive performance. The current study specifically addresses whether mate pairings that have certain personalities have higher reproductive performance than pairs that do not. We performed our study at the China Conservation and Research Center for the Giant Panda in Bifengxia, Sichuan, China and used personality scores obtained from our previous study (Chapter 3 ) to evaluate a mate pair's compatibility and reproductive performance.

\section{Methods}

\section{Study Site and Species}

We conducted personality studies on 19 female and 10 male giant pandas during the non-breeding season (May-October) of 2012 and 2013. Ages ranged from 6 to 18 years. Individuals had to be sexually mature and introduced to a member of the opposite sex on at least three different occasions for mating purposes. Housing and animal husbandry characteristics are described in our previous paper (Chapter 2). Animal care and use guidelines of the American Society of Mammalogists (Animal Care and Use Committee 1998; Assurance \#: A3675-01) were followed by all facility operators.

\section{Personality Typing}

We assessed personality of giant pandas through zookeeper personality surveys after the methods from Wielebnowski (1999), Powell and Svoke (2008), and Shepherdson et al. (2013). Personality has traditionally been assessed through direct observations carried out by one observer or by various observers that have been trained and tested for inter-observer reliability. We have previously shown that keeper surveys of panda personality have relevant and equivalent correlations with personality traits 
observed by novel object tests (Chapter 3), thus in the current study we used only personality traits obtained through keeper surveys. Though many scientists would claim that subjective animal keeper surveys are unreliable, recent research has shown that these surveys can predict real and important phenomena (Carlstead et al. 1999a; Carlstead et al. 1999b) and have been proven to be a validated as a tool for assessing animal personality (Carlstead et al. 1999b; Momozawa et al. 2003; Wielebnowski 1999).

\section{Animal Keeper Surveys}

We used keeper surveys as a tool for assessing personality in our analyses for two reasons, 1) managers are more likely to use this method because it is simple and does not involve formal behavioral assessment, 2) keeper surveys are comprehensive, integrating sources of information in many different contexts over years, whereas novel object tests assess personality only at one point in time. We conducted interviews with the primary keepers for each panda and had them complete a survey that included 23 behavioral adjectives (Chapter 3, Table 3.1). We considered the keeper who spent most time with the panda as the "primary" rater and used his/her responses in all data analyses. The 23 adjectives on the keeper survey were rated on a 10-point Likert scale (see Chapter 3). Likert scales are commonly used in surveys and typically ask participants to score a trait from 1 to 10 with a 1 indicating a low score and a 10 indicating a high score. Before rating the keepers were given an explanation of the rating procedure and the definitions of given adjectives. Keepers were instructed to score the panda compared to all pandas they have ever known.

\section{Mating Procedure}

We define mate pairings as the introduction of a specific male to a specific female 
for the purpose of breeding and mate dyads as a specific male and female giant panda pair $(\mathrm{N}=44)$. In the wild and captivity, breeding season for the panda is from February to May. We began assessing mate pairing of adult giant pandas in mid-February and continued until the end of April in 2012 and 2013 (we had two females enter estrus in May over the course of the study). The assignment of individuals to mate pairs was based on a captive breeding and genetic management plan determined by breeding managers and designed to maximize genetic health of the captive population. Mating procedures utilized by the CCRCGP are described in Chapter $2 \& 3$ ). We monitored mating success (i.e. copulation occurred with intromission) or failure and cub production $(1,0)$ of a mating pair.

\section{Data Analysis}

Principal component analysis was run on the raw data scores of the 23 adjectives obtained from keeper surveys to reduce variables into a smaller set of variables that were uncorrelated and therefore considered distinctively unique (see Chapter 3). PCA was carried out on a Varimax rotation. Scree plots were examined and components above the elbow were retained for interpretation and were labeled according to the two variables that showed highest positive loading for this component. The resultant PCA components were: 1) aggressive, 2) playful-clever, 3) fearful, and 4) stereotypical-excitable.

Giant pandas were ranked 'high' or 'low' within each PCA component based on the mean score within a sex (Table 4.1). For example, males that were ranked 'high' on the aggressive PCA component were above the mean of all males on the aggressive PCA component and males labeled 'low' were below the mean. We compared how well a mate dyad matched on personality via three separate, but related, analyses; 1) pair 
similarity/dissimilarity based on personality, 2) pair personality combinations, and 3) relative male and female personality within a pair.

To analyze a mate dyads similarity based on personality, we scored all mate dyads as similar or dissimilar. Mate dyads within the similar group were composed of males and females that were both ranked 'low' (L-L) or both ranked 'high' (H-H) on PCA component scores. Mate dyads within the dissimilar group were composed of males and females that were ranked in opposition to each other on 'low' and 'high' PCA component scores; the female was 'low' and the male was 'high' (L_H) or the female was 'high' and the male was 'low' (H_L). Because similar and dissimilar groupings resulted in mate dyads whose personalities could be substantially different (i.e. $L_{-} L$ and $\mathrm{H} \_\mathrm{H}$ were combined into the 'similar' category), we further analyzed mate dyad personality combinations by grouping mate dyads based on their personality combinations resulting in four categories for each PCA component: 1) both the male and female in a mate dyad were ranked 'low' (L_L), 2) the female was ranked 'low' but the male was ranked 'high' $\left.\left(L_{-} H\right), 3\right)$ the female was ranked 'high' but the male was ranked 'low' (H_L), and 4) both the male and female were ranked 'high' (H_H). Here and throughout the text and tables we designated the female ranking first and the male ranking second. In addition, because 'low' and 'high' was determined from scores above the mean ('high') or below the mean ('low') within sexes, theses categories did not necessarily give a relative score (i.e. whether the a male was lower or higher than a female on a given PCA component), thus, we also categorized pairings based on the male's PCA score in reference to the female's score. This relative categorization resulted in two groups: 1) males had lower scores than females on the PCA component, and 2) males had higher scores than females on the PCA 
component.

In addition to analyzing the four component scores, we also applied the three different analyses described above to raw keeper personality ratings obtained from the surveys. Pandas were ranked into 'high' (keeper rating of 6-10) or 'low' (keeper rating of 1-5). We grouped mate dyads based on their behavioral combinations for keeper ratings as described above resulting in: 1) dissimilar versus similar groups, 2) the four categories listed above (L_L, L_H, H_L, and H_H), and 3) the male's rank relative to the female's rank groups. If the male and female were ranked exactly the same by keepers (e.g. female and male were both ranked " 5 " on 'shy') the pair was excluded from analysis on that personality trait because they could not be categorized into any of the groupings described above. We analyzed rankings from keepers on 23 behavioral adjectives described in (Chapter 3, Table 3.1).

We predicted that if pairs' similarity, combinations of personalities, or relative personality contributed to mate compatibility, then certain groupings of mate pairs within personality types would have enhanced reproductive performance. We analyzed the relationship of personality to two measures of reproductive performance. The first response variable was whether a mating attempt resulted in copulation (binary response variable: yes $=1$, no $=0$ ). The second response variable was whether cubs were produced (binary response variable: yes $=1$, no $=0$ ). All data were assessed for normality and homogeneity of variance. We investigated direction and strength of relationships between personality traits and reproductive performance from related effect sizes obtained from Chi-squared tests, unpaired ANOVAs or Kruskal-Wallis tests. If significant differences were found, Tukey HSD tests were used for post hoc analyses. 
Mate dyads were considered to be statistically independent as the same male and female were never paired together more than once in our data set. Statistical significance was assumed at the level of $p<0.05$ for all tests. However, because significance testing based on critical $p$ values often does not well represent biological relevance, especially in studies of behavioral ecology (Garamszegi 2006; Nakagawa 2004), we performed post hoc tests on marginally significant results as well $(p<0.08)$. Analyses were performed in SPSS 19.0 for Mac OS X.

\section{Results}

\section{PCA Component Mate Compatibility}

Four components of the PCA personality assessment accounted for $53.3 \%$ of the observed variance (Chapter 3, Table 3.3). These components were labeled according to the variables showing the highest positive loading: aggressive (component I), playfulclever (component II), fearful (component III), stereotypical-excitable (component IV). Component I showed high positive loadings on aggressive toward conspecifics, aggressive toward humans, and solitary and high negative loadings on friendly toward conspecifics, friendly toward humans, and shy. Component II showed high positive loadings on playful, self-confident, clever, and innovative and high negative loadings on solitary. Component III showed high positive loadings on fearful of humans, fearful of conspecifics, not interested, and nervous and high negative loadings on curious, friendly towards humans, and self-confident. Component IV showed high positive loadings on stereotypical, excitable, vigilant and nervous. All negative loadings for this variable were low. 


\section{Mate dyad similarity in personality}

To determine if assortative mating in giant pandas conferred reproductive benefits, we grouped dyads into 'similar personalities' and 'dissimilar personalities' based on the PCA components. Chi-squared tests revealed that mate dyads with similar in aggressive, playful-clever, and fearful personalities were no more likely to have successful intromission or produce cubs than mate dyads with dissimilar personalities (Table 4.2). However, chi-squared tests revealed that mate dyads with dissimilar personalities in stereotypical-excitable were more likely to have successful intromission (Figure 4.1a) but not more likely to produce cubs (Figure 4.1b) than mate dyads with dissimilar personalities (Table 4.2).

\section{Mate dyad personality combinations}

Because similar and dissimilar pairs may not necessarily distinguish between the possibility of assortative mating based on other mate combinations (i.e. L_L, L_H, H_L, H_H) we grouped dyads further into these combinations to evaluate reproductive performance (Table $4.2 \& 4.3$ ). Personality combinations within the PCA aggressive component (I) showed a trend toward different intromission success and significantly different cub production between groups (Table 4.2). Tukey HD post hoc tests indicated that the L_H group on the PCA aggressive component had more successful intromissions and produced cubs more often (Figure 4.2; Table 4.3). L_H dyads had significantly more intromission successes than L_L and H_L, but not H_H mate personality combinations (Figure 4.2a). For cub production, L_H mate dyads produced significantly more cubs than L_L and H_L, but not H_H mate personality combinations (Figure 4.1b). Personality combinations within the PCA playful-clever component (II) were not 
significantly different in intromission success or cub production between $\mathrm{L}_{-} \mathrm{L}, \mathrm{L} \_\mathrm{H}$, H_L, or H_H groups so post hoc tests were not conducted (Table 4.2). Personality combinations within the PCA fearful component (III) had significantly different intromission success but not cub production between the four groups (Table 4.2). Tukey HD post hoc tests indicated the H_L dyads had significantly more intromission successes than $\mathrm{H}_{-} \mathrm{H}$, trending toward significantly more intromissions than $\mathrm{L} \_\mathrm{H}$, but was not significantly different from L_L mate personality combinations (Figure 4.3; Table 4.3). Additionally, $\mathrm{L}_{-} \mathrm{L}$ mate dyads had significantly more intromissions than $\mathrm{H} \_\mathrm{H}(p=0.03)$ but not $\mathrm{H} \_\mathrm{L}$ or $\mathrm{L} \_\mathrm{H}$ mate personality combinations. Post hoc analyses were not conducted on cub production as ANOVAs were not significant. Personality combinations within the PCA stereotypical-excitable component (I) had significantly different intromission success but not cub production between groups (Table 4.2). Tukey HD post hoc tests indicated that L_H dyads had significantly more intromission successes than $\mathrm{H} \_\mathrm{H}$ dyads, trended towards more intromission success than $\mathrm{L} \_\mathrm{L}$, but did not show a difference from $H_{-} \_$mate personality combinations (Figure 4.4; Table 4.3). Post hoc analyses were not conducted on cub production as ANOVAs were not significant.

\section{Mate dyad relative personality scores}

Because the above groupings did not give us an indication of the male and female's personality ranking relative to the opposite sex in individual pairings, we further evaluated the mate compatibility of the dyads on PCA components by grouping the dyads into mate pairings where the male's PCA component scores was lower than the female's PCA component score and dyads where the male's PCA component score was higher. 
This grouping allowed us to compare aggressive scores of giant pandas relative to their mates. Chi-square tests revealed that dyads composed of males with higher aggressive PCA component scores than females had significantly more intromission success and cub production than dyads composed of males with lower aggressive PCA component scores than females (Figure 4.5; Table 4.2). Relative differences in male-female personality scores for playful-clever, fearful, and stereotypical-excitable PCA component scores were not significantly associated with measures of reproductive performance.

\section{Keeper Rating Mate Compatibility}

We further analyzed mate dyad compatibility based on raw keeper ratings. Only four personality keeper scores showed significant differences between $L_{-} L, L \_H, H \_L$, and $\mathrm{H}_{-} \mathrm{H}$ dyad groupings, 'active', 'calm', 'aggressive toward conspecifics', and 'innovative'. All other keeper rating scores showed no significant differences between groups (See Appendix D). Mate dyads divided by 'active' keeper ratings were significantly different between groups in intromission success and cub production with L_H mate pairings showing more successful intromission and producing cubs more often (See Appendix D). There was also a significant difference between mate dyad groups based on the 'calm' keeper ratings in both intromission success and cub production. Neither the 'active' or 'calm' keeper rating loaded on any of our PCA components. Pairs that were ranked as L_H 'aggressive toward conspecifics' keeper ratings were significantly different from other groups in intromission success and cub production showing a similar trend to the aggressive component as described above (See Appendix D). The keeper rating 'aggressive toward conspecifics' positively loaded on the aggressive PCA component score. L_L dyads based on 'innovative' keeper rating had 
more successful intromissions and produced cubs more often (See Appendix D). The keeper rating 'innovative' positively loaded on the playful PCA component score as explained above.

\section{Discussion}

For clarity, our findings on personality-types are summarized in Table 4.4 based on statistically significant differences found between groups. Giant pandas appear to be mating assortatively on aggressive, fearful, and stereotypical-excitable personality traits. Measures of reproduction were higher when high aggression males were mated with low aggression females and when high stereotypical-excitable males were mated to low stereotypical-excitable females (negative-assortatively mating with regard to personality traits). By contrast, intromission rates were higher when males low in fearfulness were mated to females low or high in fearfulness (both positive and negative assortative mating).

For aggressiveness, there was no difference between mate dyads when we grouped pairs into 'dissimilar' and 'similar' aggressive personalities. However, mate dyads where females were ranked 'low' on aggressiveness and males were ranked 'high' (H_L mate dyads) showed significantly higher intromission success and cub production than all other combinations of mate dyads ranked on this personality characteristic. This relationship was also evident when we ranked individuals as 'low' or 'high' relative to the opposite sex conspecific paired for mating (i.e. mate dyads with males ranked higher than females showed more reproductive performance than the reciprocal). Additionally, pairs rated $\mathrm{L}_{-} \mathrm{H}$ on the raw keeper rating of 'aggressive toward conspecifics' had more 
intromission success and produced more cubs. Taken together, these findings strongly suggest that giant pandas are assortatively mating based on dissimilar aggressive personalities and aggressiveness relative to a mating partner. This relative aggressiveness is important for reproductive performance measurements and our data suggest males must be ranked higher than females in aggression. Our recent research found that aggressiveness is an important component of long-term cub production for female giant pandas (Chapter 3). In this previous study, females that were rated low by keepers as 'aggressive toward conspecifics' and on the aggressive PCA component score produced more cubs long-term than females rated high on these aggressiveness scores (Chapter 3). This finding is in agreement with our current study as mate dyads composed of females with low scores produced more cubs, however, it also expanded on our knowledge of fearfulness within specific mating pairs. Both studies are in agreement with Ellis et al. (2006) who found that aggressiveness was an important predictor of long-term breeding success.

The current study allowed more differentiation from our last study (Chapter 3) through evaluating the effect of personality on reproductive performance of a specific mate pairing. We were also able to test Ellis et al.'s (2006) claim that "the presence of only [one] assertive male or female (but not both) did not enhance reproductive success". Our research suggests this may not be the case, instead, pairs where males were more aggressive than females had the highest reproductive performance. However, mate dyads where males and females were both 'high' on aggressiveness ratings had the second highest intromission success (though not significant) and may also show increased reproductive performance with an increase in sample size. Our findings 
parallel wild giant panda ecology. In the wild, male giant pandas compete aggressively for females and the dominant male retains breeding rights to the female (Nie et al. 2012b; Schaller et al. 1985). Aggression is often central to establishing dominance (Ellis 1995) in other species such as coho salmon (Fleming \& Gross 1993), rats and mice (Blanchard et al. 1984; Dewsbury 1984), lizards (Fox 1983), and primates (DeVore 1965) and this increased aggressiveness of dominant individuals increases reproductive performance measurements. Likewise, Carlstead et al. (1999b) found a relationship between dominant and aggressive individuals and increased reproductive performance in black rhinoceros, though the relationship was opposite to our findings (i.e. dominant females paired with non-dominant males had highest reproductive performance). Aggression linked to dominance appears to exist in wild giant pandas as well. Nie et al. (2012b) found that congregating males displayed high levels of aggression and dominant males maintained high levels of aggression toward rival males throughout the competition period until copulation was successful. Surprisingly, they found no relationship between testosterone levels and dominance status in male giant pandas (Nie et al. 2012b) but in a concurrent study the same group found that in general male testosterone levels increase during mating congregations (Nie et al. 2012c). Taken together, these findings lend support to our suggestion that aggressiveness in male giant pandas is helpful for successful reproduction and may be mediated by increased testosterone during the mating season (though relative increases in testosterone to rival males does not appear to determine male dominance rank). Future studies should investigate levels of testosterone in captive male giant pandas as they approach breeding season and within the breeding season around mate introductions. During mate introductions, captive male giant pandas are 
denied access to rival males, which may be reducing their overall aggressiveness during important reproductive periods. Limiting intra-sexual competition between males could inadvertently lower reproductive performance through lowering aggression levels. Future studies should investigate how male-male competition effects aggression and subsequent reproductive performance.

Hyper-aggression during mate pairings has been implicated in reproductive failure in giant panda captive breeding programs (Ellis et al. 2006). Zhang and colleagues (2004a) suggest that when male giant pandas exhibit hyper-aggression during mate introductions those mating attempts often fail. Combining results from our previous and current study indicates that males must be ranked high on levels of aggression in order to be successful breeders and that females also must show an appropriate level of aggression for successful cub production but too much is detrimental to the mate pairing (see Figure 2; $H_{-} H$ pairs had lower success than $L_{-} L$ and $L_{-} H$ ). Ellis et al. (2006) suggest that there is probably some 'optimal' level of aggression for giant panda mating pairs. The average aggressive score for female giant pandas in the $\mathrm{L} \_\mathrm{L}$ groups was $-0.31 \pm 0.31, \mathrm{~L} \_\mathrm{H}$ group was $-0.59 \pm 0.60, \mathrm{H} \_L$ groups was $0.19 \pm 0.13$, and $\mathrm{H} \_\mathrm{H}$ group was $0.67 \pm 0.48$. The average aggressive score for male giant pandas in the $\mathrm{L} \_\mathrm{L}$ groups was $-1.2 \pm 0.19, \mathrm{~L} \_\mathrm{H}$ group was $0.48 \pm 0.62, \mathrm{H} \_L$ groups was $1.10 \pm 0.24$, and $\mathrm{H} \_\mathrm{H}$ group was $0.75 \pm 0.84$. H_L and $\mathrm{H} \_\mathrm{H}$ groups had the highest reproductive performance and because these numbers spanned the whole range of aggression scores for females (-0.59-0.67) this would indicate that there is no 'optimal' level of overall aggressiveness for females. However, our results suggest that males should be $>0.24$ on our aggressive component for optimal reproductive performance and males should always be anywhere from 0.1- 
0.51 above females on our aggressive component score.

We have recently shown that fearfulness was an important predictor of long-term reproductive performance in giant pandas (Chapter 3). While the current study looked specifically at annual mate pairings in the year personality tests were conducted, our previous study used these same personality factors to determine long-term reproductive performance. Both studies were in agreement that fearfulness negatively impacted reproductive performance measurements both of long-term and on an individual mating basis. Reduced fearfulness was an important indicator of intromission success for both male and female giant pandas. Pairs ranked low on fearfulness (i.e. bolder male and female pairs) had the highest reproductive performance followed closely by pairs where males were ranked low on fearfulness but females were ranked high (H_L mate dyads). Similar to aggression levels, this implicates male fearfulness as being more important than female fearfulness in leading to reproductive failure. However, whereas intromission success seemed to be determined by both male and female fearfulness, cub production was only affected by fearfulness of female giant pandas. Female giant pandas that produced fewer cubs long-term (or none at all) scored significantly higher on 'fear towards human' scores and on the fearful PCA component score (Chapter 3). This indicates that fearfulness in female giant pandas may be a very important predictor of long-term reproductive performance due to factors discussed previously that may impact fertilization and pregnancy rates (e.g. increased cortisol levels with increased fearfulness leading to lower ovulation and pregnancy rates in females and sperm production in males; Chapter 3).

Our findings are in agreement with Powell and Svoke (2008) who found female 
giant pandas ranked as shy or timid have poorer socio-sexual performance than bolder, confident females. However, their study did not follow giant panda reproductive performance through to cub production, thus, our current study extends the findings through to cub production. Cub production was not affected by fearfulness when we looked at reproductive performance in terms of compatibility of mates but did impact a female's overall long-term cub production (Chapter 3). This is similar to studies in other species, such as cheetahs, where non-breeders were more tense-fearful than breeders (Wielebnowski 1999). In addition, it has recently been shown that fearful wild great tits prioritize survival over reproductive investment (Cole \& Quinn 2014), suggesting that personality traits may reflect life-history variation in risk responsiveness. As Powell and Svoke (2008) suggest, efforts to reduce shyness/fearfulness may indeed increase reproductive performance but we would caution against such efforts as the long-term effects of altering personality has not been well studied in giant pandas. Current research suggests that differences in captive and wild population personalities may influence the likelihood that individuals can be reestablished in the wild (Elsbeth McPhee 2004; McDougall et al. 2006; Smith \& Blumstein 2008) and, thus, the repercussions of attempting to alter personalities of giant pandas or focusing breeding efforts on cultivating certain personalities are not well understood. If managers attempt to alter personality profiles of individual giant pandas this could affect the overall reproductive performance of a captive population and risks an evolutionary shift in the captive stock genetics (McDougall et al. 2006). More research needs to be conducted on genetic correlates with personality and learned components of personality to more fully inform a discussion on possible repercussions of altering personality traits in captivity to increase 
reproductive performance. However, breeding managers could incorporate personality profiles to alter breeding environments such that certain personality types would still reproduce, thus, maintaining behavioral diversity in captivity. Though cub production is a more reliable indicator of fitness than intromission success, we suggest that offspring in the current study should be reassessed for survivorship and breeding ability at adulthood similar to studies done on house mice (Drickamer et al. 2000a; Gowaty et al. 2003) to get a better indicator of the consequences of personality on male and female giant panda long-term fitness.

Giant panda mate dyads that were ranked as dissimilar on the stereotypicalexcitable component had more successful intromissions but not more cubs. This finding suggests that giant pandas may be negatively assortatively mating based on this personality characteristic. Similar to the aggressive component, mate dyads composed of females ranked 'low' on the stereotypical-excitable component and males ranked 'high' (L_H dyads) had increased intromission success and produced more cubs. To our knowledge, this is the first study that suggests giant pandas may be differentiating between stereotypical-excitable mate partners and non-stereotypical-excitable mate partners. Recent studies have implicated increased stereotypical behavior in both increased and decreased reproductive performance implying that the directional effect of this factor may be species dependent and sex dependent. For example, a study evaluating enriched and non-enriched male mink found that highly stereotypic males gained fewer copulations than their non-stereotypic counterparts (Díez-León et al. 2013). Likewise, a similar study showed in striped mice (Rhabdomys) that stereotypic females, but not stereotypic males, had more total number of offspring than nonstereotypic 
females (Jones et al. 2010). For giant pandas, the relationship seems to be the opposite in that higher stereotypical-excitable males had more intromission and cub production than lower stereotypical-excitable males. It is possible that males labeled as highly stereotypical-excitable may increase their sexual behaviors (including aggressive behaviors) during pre-mate observations and mate pairings (exciting events) ultimately resulting in a perceived, or real, increase in interest toward the potential female mate. Testosterone and cortisol levels in conjunction with more fine-tuned behavioral measurements of stereotypical behavior and excitability during mate introductions would help further inform our findings.

The relationship we found between stereotypical males and higher reproductive performance is harder to interpret with the current data set. The presence of stereotypical behaviors have been implicated as an indicator of poor welfare in captivity (Mason \& Latham 2004). However, the literature is divided on this issue with some studies suggesting stereotypies indicate poor welfare (Garner \& Mason 2002; Mason 1991; Rushen et al. 1993) and other studies pointing out that stereotypies do not always indicate poor welfare and may be a coping mechanism for unnatural and/or suboptimal environments (Wechsler 1995). For example in farmed mink stereotypic animals had lower corticostereoid levels than animals that exhibited no stereotypies (EC 2001; Mason \& Latham 2004). Giant pandas often display stereotypical behavior in captivity such as pacing, pirouetting, tongue flicking, and vocal stereotypes (Liu et al. 2003; Swaisgood et al. 2001). Previous studies have proposed that stereotypic behavior patterns in captive pandas are a compensation for, or adaptation to, a restricted, unenriched environment (Mason 2006). The typical method of determining whether stereotypical behavior is an 
indicator of poor welfare is through measuring corticosteroid levels as a proxy for increased stress and correlating this with stereotypical behavior. To our knowledge there is only one study evaluating the links between corticosteroids and stereotypical behavior in giant pandas. Liu et al. (2006) found a correlation between cortisol levels and stereotypical behavior during estrus in female giant pandas. We have no way of predicting whether males high on the stereotypical-excitable personality component had higher or lower cortisol than nonstereotypical males. However, there are two possible explanations for higher reproductive performance if either of these options exist. If males in our study that are high on the stereotypical-excitable personality component had low cortisol levels compared to nonstereotypical males this would indicate that stereotypical behavior may serve as a coping mechanism in giant pandas reducing cortisol to levels acceptable for increased reproductive performance. However, if stereotypical-excitable males have higher cortisol levels than nonstereotypical males this would indicate that increased cortisol levels confer a reproductive benefit in giant pandas. Future studies should examine correlations between cortisol levels and stereotypical behavior both outside and within the breeding season. To further elucidate the effects of stereotypical-excitable personality characteristics on sexual motivation and reproductive performance measurements further studies must be performed specifically on stereotypical and excited behaviors during pre-mating behavioral evaluations, behaviors in mate introductions, intromission success, and cub production.

The results from this study have significant implications for captive breeding programs of endangered species and suggest that relative personality traits of males and females in giant panda mate dyads affect reproductive performance of the pair. Our 
findings indicate that giant pandas pairs are not assortatively mating based on personality similarities, but rather based on dissimilarities for aggression and stereotypical-excitable personality traits. Mating dyads composed of males 'high' on these personality traits and females ranked 'low' had the highest reproductive performance out of all possible mate combinations. Conversely, the fearfulness component showed the opposite relationship with only mate dyads where both sexes were ranked low resulting in higher reproductive performance. In conjunction with our previous study (Chapter 3) it appears that behavioral personality assessments may allow breeding managers to more accurately predict an individual's ability to reproduce in captive environments and these assessments could be used as a tool by breeding managers to increase reproductive performance in captive settings. $100 \%$ of L_H stereotypical-excitable dyads and $80 \%$ of L_H aggressive dyads had intromission with approximately $70 \%$ of these pairings resulting in cubs. If we extend these findings to the number of mating attempts conducted each year ( 83), and assume that we could accurately pair giant pandas based on personality, $90 \%$ of these pairings ( 74$)$ would have successful intromission. The current intromission success rate is $23 \%$. Even if managers were only able to accurately pair giant pandas based on personality $50 \%$ of the time we would still see a dramatic increase in intromission success in the giant panda breeding program. Therefore, our data suggests that in addition to selecting mate pairs based on genetic compatibility, we suggest breeding managers incorporate behavioral measures of personality to alter breeding environments that would maximize the reproductive performance of the captive breeding program. 


\section{Acknowledgments}

We thank the CCRCGP giant panda keepers, researchers and staff for all their help and support. This work was supported by an Animal Behavior Society Student Research Grant, an Oregon Zoo Future for Wildlife Grant, PDXWildlife, and a Portland State University’s Forbes Lea Grant. We thank our hard working interns, Arina Woolery, Kim Render, Stephanie McMahon, Amelie LeRoy, and Grace Russell - without their hard work none of this would have been accomplished. 
Table 4.1. Means and ranges for female and male giant pandas ranked 'low' and 'high' based on principal components obtained from keeper ratings of giant panda personalities.

Rank

\begin{tabular}{|c|c|c|}
\hline Variable & Low & High \\
\hline \multicolumn{3}{|c|}{ Aggressive Component } \\
\hline \multicolumn{3}{|c|}{ Females } \\
\hline Mean & -0.49 & 0.50 \\
\hline Range & $-1.80--0.05$ & $-0.01-1.59$ \\
\hline \multicolumn{3}{|l|}{ Male } \\
\hline Mean & -1.13 & 0.70 \\
\hline Range & $-1.49--0.84$ & $0.09-2.30$ \\
\hline \multicolumn{3}{|c|}{ Playful Component } \\
\hline \multicolumn{3}{|c|}{ Females } \\
\hline Mean & -0.55 & 0.98 \\
\hline Range & $-1.49--0.3$ & $0.31-1.81$ \\
\hline \multicolumn{3}{|l|}{ Male } \\
\hline Mean & -0.33 & 1.06 \\
\hline Range & $-1.15--0.13$ & $0.55-2.18$ \\
\hline \multicolumn{3}{|c|}{ Fearful Component } \\
\hline \multicolumn{3}{|c|}{ Females } \\
\hline Mean & -0.66 & 1.15 \\
\hline Range & $-1.38--0.07$ & $0.42-1.95$ \\
\hline \multicolumn{3}{|l|}{ Male } \\
\hline Mean & -1.05 & -0.26 \\
\hline Range & $-1.38--0.86$ & $-0.38-0.33$ \\
\hline \multicolumn{3}{|c|}{ Stereotypical Component } \\
\hline \multicolumn{3}{|c|}{ Females } \\
\hline Mean & -1.00 & 0.62 \\
\hline Range & $-2.08--0.56$ & $0.25-1.20$ \\
\hline \multicolumn{3}{|l|}{ Male } \\
\hline Mean & -0.75 & 0.53 \\
\hline Range & $-1.58--0.16$ & $0.30-1.26$ \\
\hline
\end{tabular}


Table 4.2. Statistical analyses of reproductive performance for mate dyads of giant pandas grouped by personality similarity, combinations of personalities, and relative personalities based on component (PCA) scores for aggressive, playful-clever, fearful, and stereotypical-excitable. Bold numbers indicate significant difference between PCA personality groups at $p<0.05$

\begin{tabular}{|c|c|c|c|c|}
\hline \multirow[b]{2}{*}{ Variable } & \multicolumn{4}{|c|}{ PCA Component } \\
\hline & Aggressive & Playful-clever & Fearful & $\begin{array}{c}\text { Stereotypical- } \\
\text { Excitable }\end{array}$ \\
\hline \multicolumn{5}{|l|}{ Similarity in personalities } \\
\hline Intromission success & $1.83(0.18)^{\mathrm{a}}$ & $0.14(0.71)^{\mathrm{a}}$ & $1.22(0.27)^{\mathrm{a}}$ & $6.79(0.009)^{\mathrm{a}}$ \\
\hline Cub production & $0.55(0.46)^{\mathrm{a}}$ & $0.36(0.55)^{\mathrm{a}}$ & $0.02(0.88)^{\mathrm{a}}$ & $1.08(0.30)^{\mathrm{a}}$ \\
\hline \multicolumn{5}{|l|}{ Combinations of personalities } \\
\hline Intromission & $2.47(0.06)^{\mathrm{b}}$ & $0.90(0.83)^{b}$ & $11.66(0.009)^{c}$ & $7.51(0.05)^{c}$ \\
\hline Cub production & $3.75(0.05)^{b}$ & $2.92(0.40)^{\mathrm{b}}$ & $2.06(0.13)^{\mathrm{b}}$ & $0.63(0.60)^{b}$ \\
\hline \multicolumn{5}{|l|}{ Relative personalities } \\
\hline Intromission & $5.13(0.02)^{\mathrm{a}}$ & $0.83(0.77)^{\mathrm{a}}$ & $1.66(0.20)^{\mathrm{a}}$ & $0.03(0.86)^{\mathrm{a}}$ \\
\hline Cub production & $3.95(0.05)^{\mathrm{a}}$ & $0.07(0.80)^{\mathrm{a}}$ & $1.91(0.17)^{\mathrm{a}}$ & $0.18(0.67)^{\mathrm{a}}$ \\
\hline
\end{tabular}

${ }^{a}$ Chi-squared tests; $\chi^{2}$ ( $p$ value)

${ }^{\mathrm{b}}$ ANOVA; $F_{3,26}$ ( $p$ value)

${ }^{\mathrm{c}}$ Kruskall-Wallis; $\chi^{2}$ ( $p$ value) 
Table 4.3. Values and measures of reproductive performance for mate dyads of giant pandas grouped by personality combinations based on principal component (PCA) scores for aggressive, playful-clever, fearful, and stereotypical-excitable. Female and male component scores are reported in means \pm standard deviation. Bold numbers indicate groups with higher reproductive performance measurements. Superscripts indicate differences between groups at $p<0.05$; different superscripts groups that were statistically different from each other and similar superscripts groups that were not.

\begin{tabular}{|c|c|c|c|c|}
\hline \multirow[b]{2}{*}{ Variable } & \multicolumn{4}{|c|}{ Mate Dyad Group } \\
\hline & $\mathrm{L} \_\mathrm{L}$ & L_H & H_L & $\mathrm{H} \_\mathrm{H}$ \\
\hline \multicolumn{5}{|l|}{ Aggressive PCA Component Score } \\
\hline Total mate pairings & 7 & 11 & 6 & 6 \\
\hline Total successful intromissions & $2^{\mathrm{a}}$ & $9^{b}$ & $2^{\mathrm{a}}$ & $4^{\mathrm{a}, \mathrm{b}}$ \\
\hline Cubs produced & $1^{\mathrm{a}}$ & $7^{\mathrm{b}}$ & $1^{\mathrm{a}}$ & $3^{\mathrm{a}, \mathrm{b}}$ \\
\hline Cubs maternally reared & 0 & 7 & 1 & 3 \\
\hline Female & $-0.31 \pm 0.31$ & $-0.59 \pm 0.60$ & $0.19 \pm 0.13$ & $0.67 \pm 0.48$ \\
\hline Male & $-1.2 \pm 0.19$ & $0.48 \pm 0.62$ & $-1.10 \pm 0.24$ & $0.75 \pm 0.84$ \\
\hline \multicolumn{5}{|c|}{ Playful-Clever PCA Component Score } \\
\hline Total mate pairings & 7 & 9 & 6 & 8 \\
\hline Total successful intromissions & 3 & 5 & 4 & 5 \\
\hline Cubs produced & 2 & 4 & 1 & 5 \\
\hline Cubs maternally reared & 2 & 4 & 0 & 5 \\
\hline Female & $-0.45 \pm 0.38$ & $-0.55 \pm 0.41$ & $0.97 \pm 0.59$ & $0.98 \pm 0.50$ \\
\hline Male & $-0.40 \pm 0.34$ & $0.99 \pm 0.52$ & $-0.26 \pm 0.09$ & $0.94 \pm 0.55$ \\
\hline \multicolumn{5}{|l|}{ Fearful PCA Component Score } \\
\hline Total mate pairings & 4 & 6 & 9 & 11 \\
\hline Total successful intromissions & $4^{\mathrm{a}}$ & $2^{\mathrm{a}, \mathrm{b}}$ & $8^{a}$ & $3^{\mathrm{b}}$ \\
\hline Cubs produced & 3 & 1 & 5 & 3 \\
\hline Cubs maternally reared & 3 & 1 & 5 & 2 \\
\hline Female & $-0.54 \pm 0.23$ & $-0.74 \pm 0.41$ & $1.31 \pm 0.56$ & $\begin{array}{c}1.01 \pm 0.60 \\
-0.26 \pm\end{array}$ \\
\hline $\begin{array}{l}\text { Male } \\
\text { Stereotypical-excitable PCA } \\
\text { Component Score }\end{array}$ & $-1.03 \pm 0.20$ & $-0.23 \pm 0.20$ & $-1.03 \pm 0.20$ & 0.02 \\
\hline Total mate pairings & 8 & 7 & 4 & 12 \\
\hline Total successful intromissions & $3^{\mathrm{a}}$ & $7^{\mathrm{b}}$ & $2^{a, b}$ & $5^{\mathrm{a}}$ \\
\hline Cubs produced & 3 & 4 & 1 & 4 \\
\hline Cubs maternally reared & 3 & 4 & 1 & 3 \\
\hline Female & $-1.04 \pm 0.60$ & $-1.01 \pm 0.54$ & $0.73 \pm 0.29$ & $0.58 \pm 0.27$ \\
\hline Male & $-0.58 \pm 0.61$ & $0.57 \pm 0.38$ & $-0.62 \pm 0.41$ & $0.47 \pm 0.15$ \\
\hline
\end{tabular}


Table 4.4. Significant findings of increased reproductive performance for mate dyads of giant pandas grouped by personality similarity, combinations of personalities, and relative personalities based on component (PCA) scores for aggressive, playful-clever, fearful, and stereotypical-excitable. ' $i$ ' indicates intromission success was higher and ' $c$ ' indicates cub production was higher.

\begin{tabular}{|c|c|c|c|c|}
\hline \multirow[b]{2}{*}{ Variable } & \multicolumn{4}{|c|}{ PCA Component } \\
\hline & Aggressive & Playful-clever & Fearful & $\begin{array}{c}\text { Stereotypical- } \\
\text { Excitable }\end{array}$ \\
\hline \multicolumn{5}{|l|}{ Similarity in personalities } \\
\hline Intromission success & - & - & - & $\mathrm{i}$ \\
\hline Cub production & - & - & - & - \\
\hline \multicolumn{5}{|l|}{ Combinations of personalities } \\
\hline $\mathrm{L}_{-} \mathrm{L}$ & - & - & $\mathrm{i}$ & - \\
\hline $\mathrm{L}_{-} \mathrm{H}$ & $\mathrm{i}, \mathrm{c}$ & - & - & $\mathrm{i}, \mathrm{c}$ \\
\hline H_L & - & - & $\mathrm{i}$ & - \\
\hline H_H & - & - & - & - \\
\hline \multicolumn{5}{|l|}{ Relative personalities } \\
\hline Male lower & - & - & - & - \\
\hline Male higher & $\mathrm{i}, \mathrm{c}$ & - & - & - \\
\hline
\end{tabular}


a)

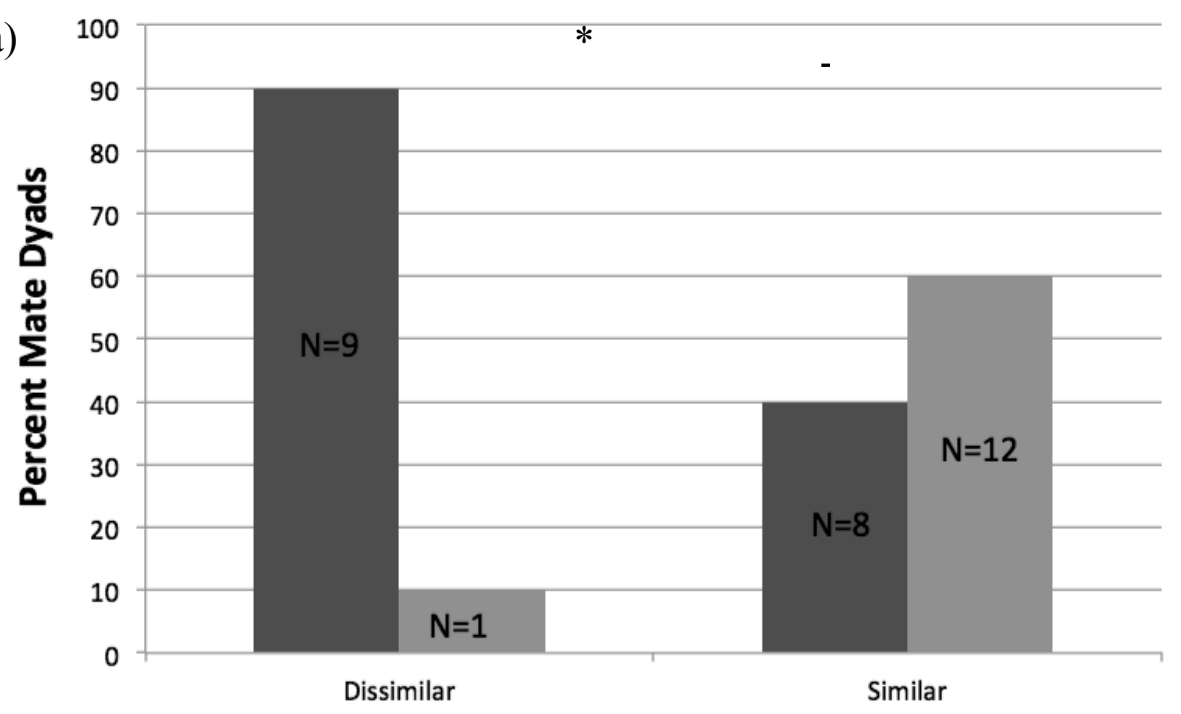

b)

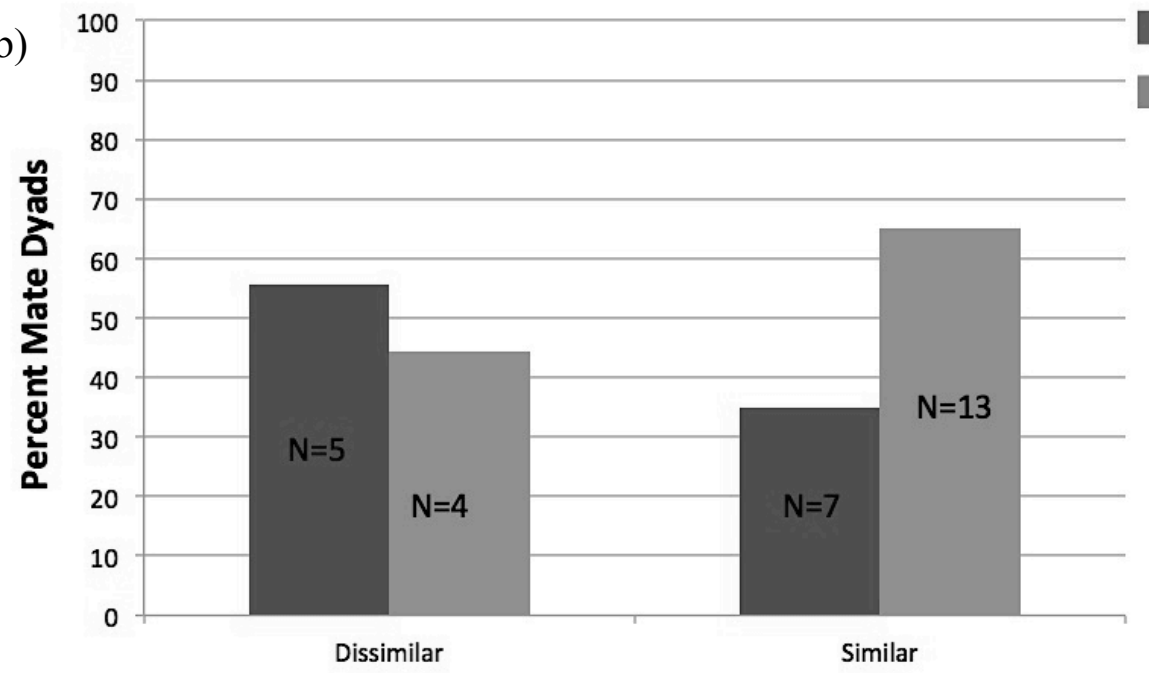

Mate Dyad Stereotypical Component Score
Intromission

No Intromission
Cubs Produced

No Cubs Produced

Figure 4.1. The percent of mate dyads resulting in a) successful intromission and b) cub production for mate pairings composed of females and males ranked similar to each other than females and males ranked dissimilar to each other on the stereotypical PCA component scores. $N$ represents the number of mate dyads. *indicates $p \leq 0.05$ 
a)

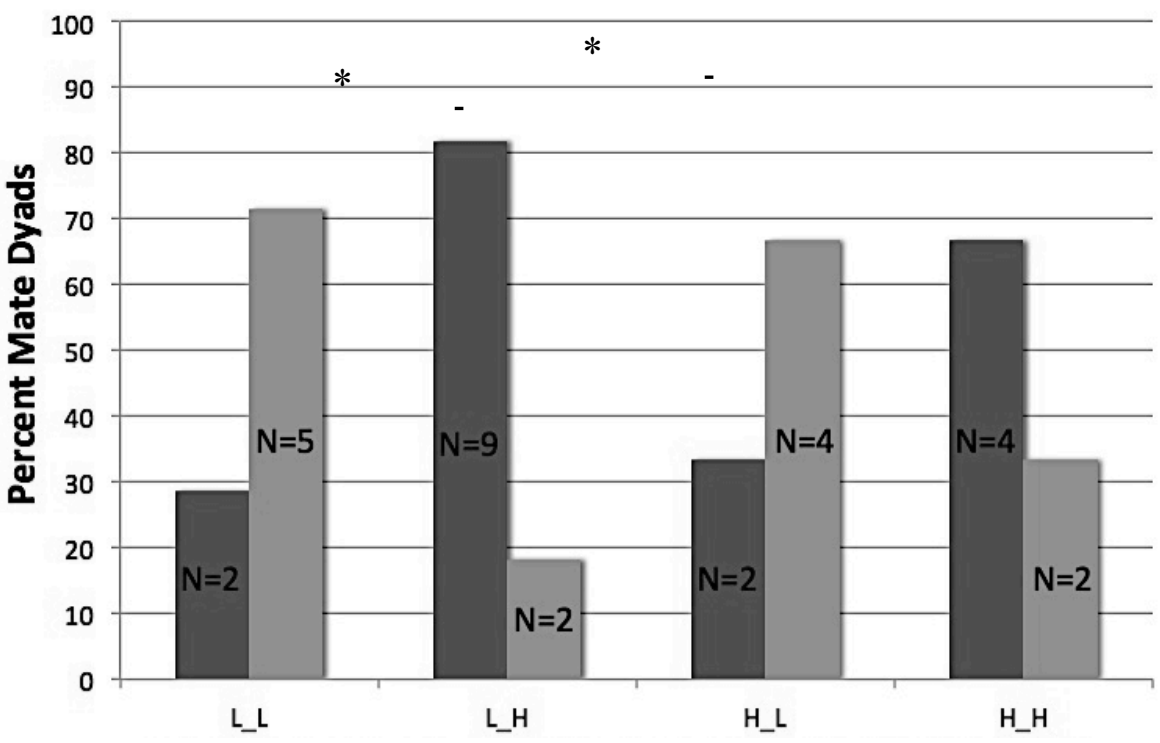

b)

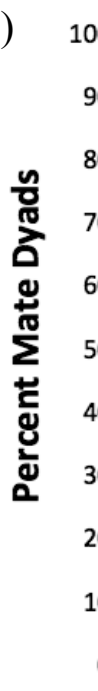

$*$

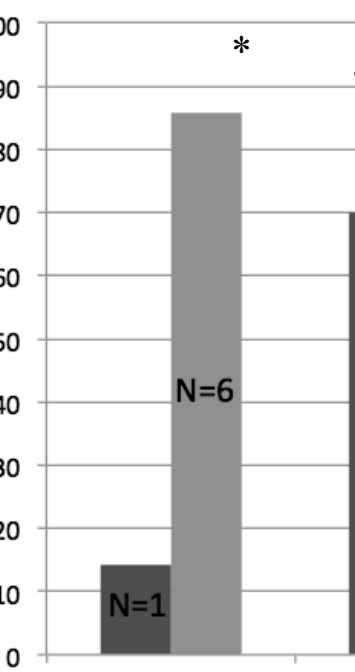

L_L

Intromission

No Intromission

Cubs Produced

No Cubs Produced

Mate Dyad Aggressive Component Compatibility Group

Figure 4.2. The percent of mate dyads resulting in a) successful intromission and b) cub production for mate pairings of females and males that were both ranked low on aggressive component scores $\left(L_{-} L\right)$, of females that were ranked low and males that were ranked high $\left(L_{-} H\right)$, of females that were ranked high and males that were ranked low $\left(H_{-} L\right)$, and of females and males that were both ranked high (H_H). $\mathrm{N}$ represents the number of mate dyads. * indicates $p \leq 0.05$ 
a)

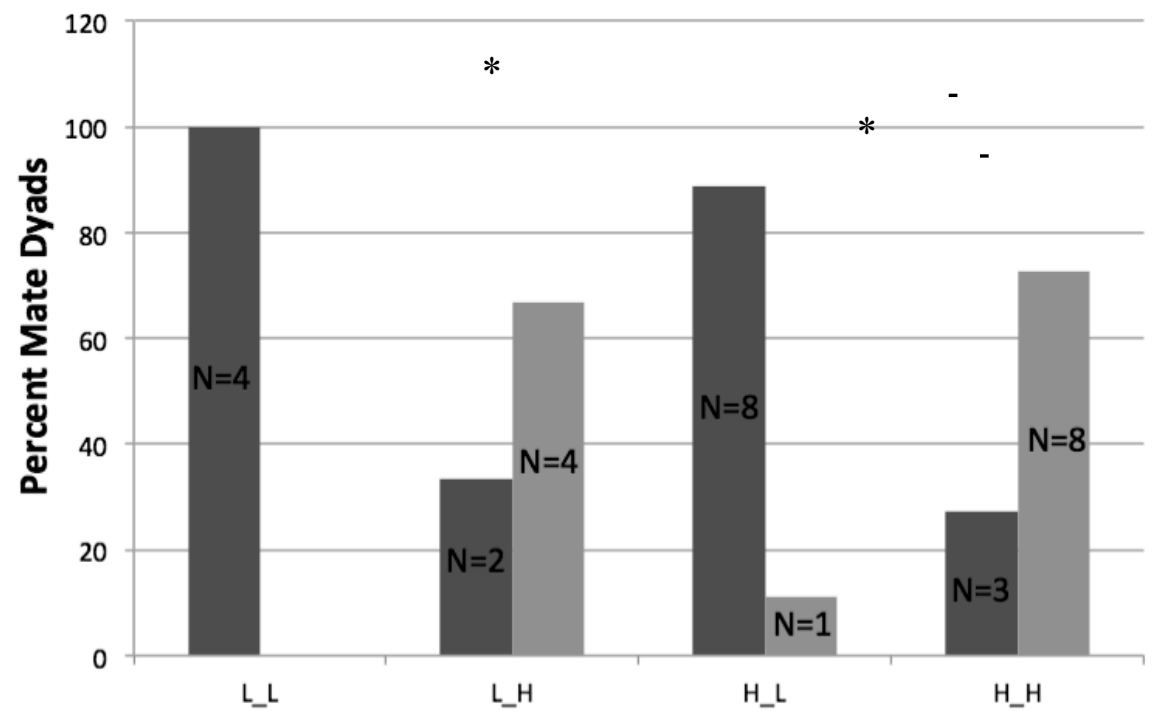

b)

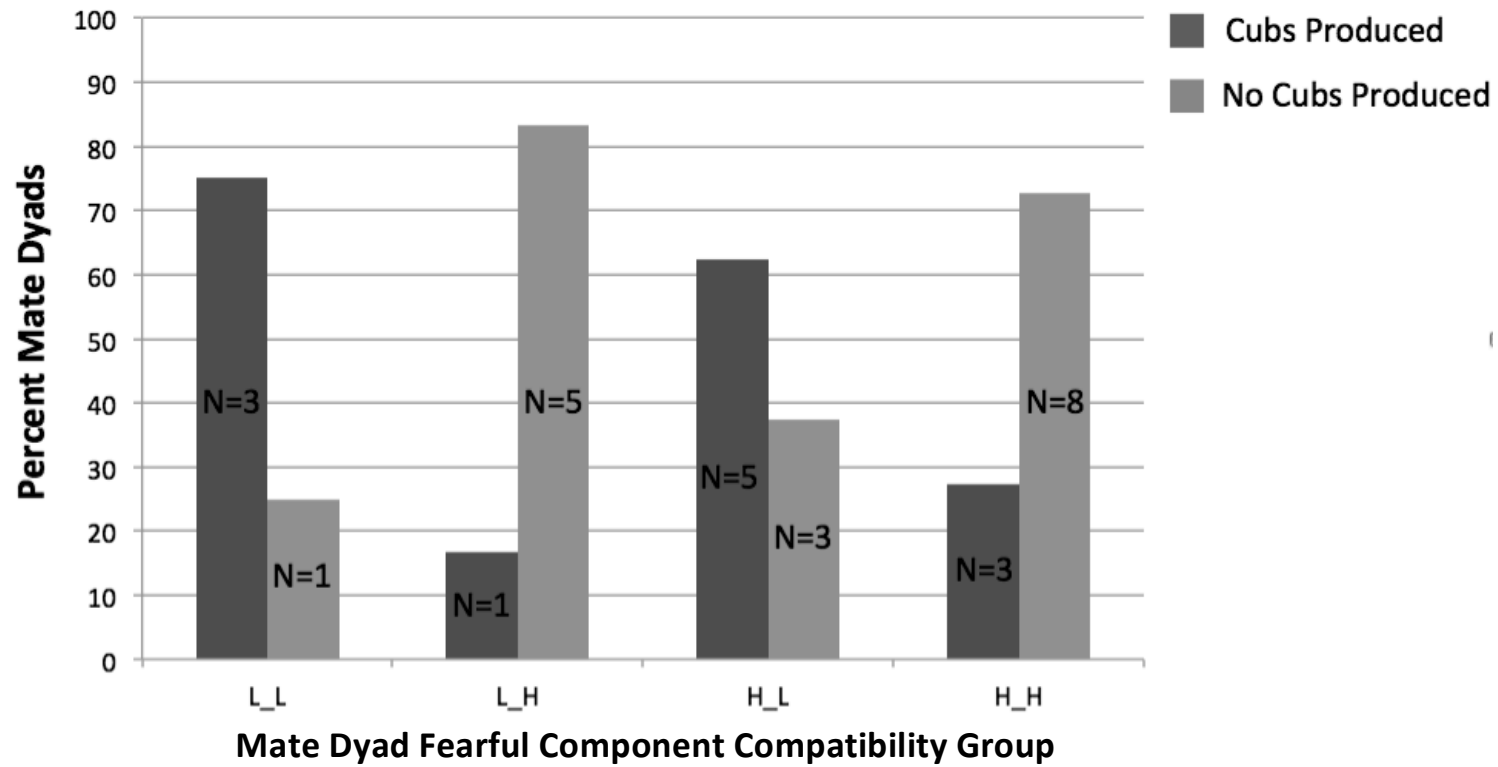

Figure 4.3. The percent of mate dyads resulting in a) successful intromission and b) cub production for mate pairings composed of females and males that were both ranked low on fearful component scores $\left(L_{-} L\right)$, of females that were ranked low and males that were ranked high $\left(L_{-} H\right)$, of females that were ranked high and males that were ranked low $\left(H_{-} L\right)$, and of females and males that were both ranked high $\left(\mathrm{H}_{-} \mathrm{H}\right)$. $\mathrm{N}$ represents the number of mate dyads. *indicates $p \leq 0.05$ 


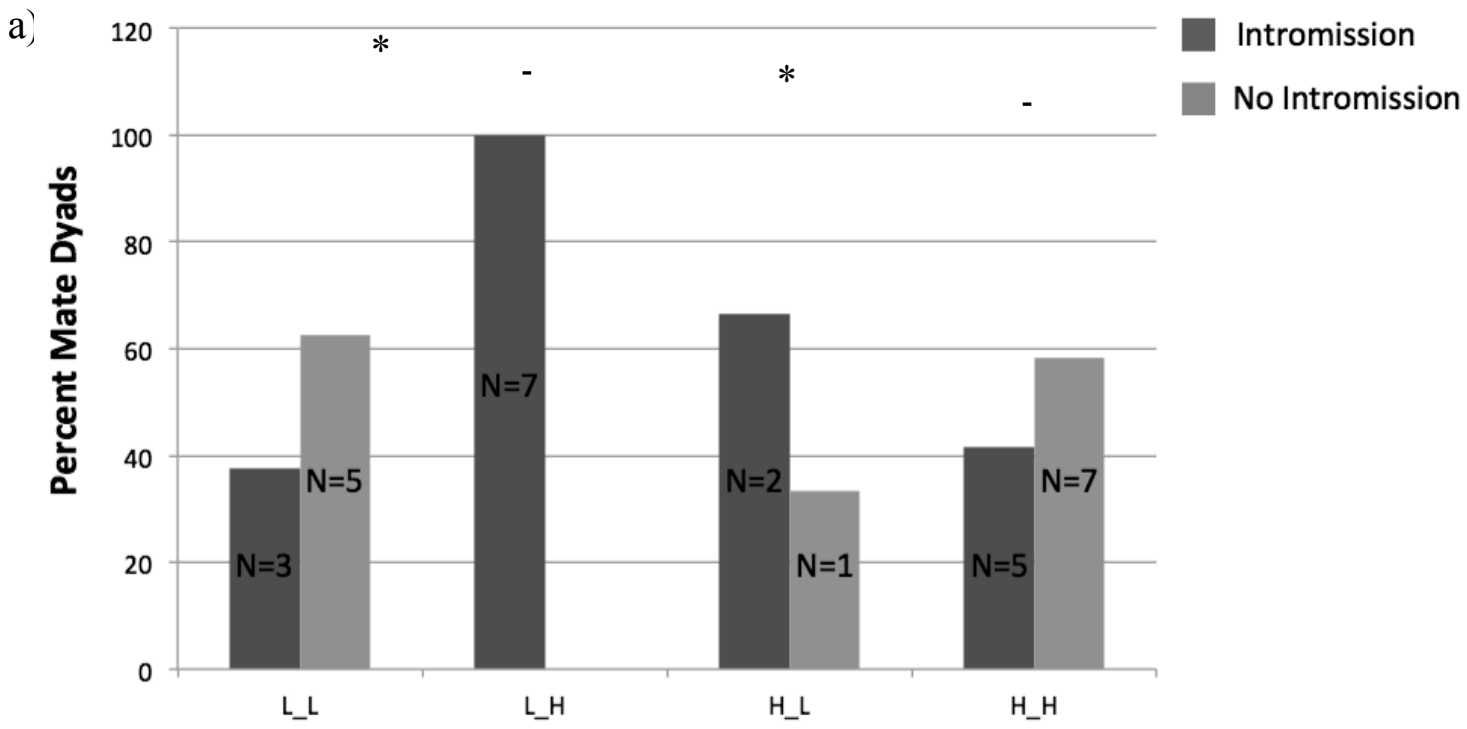

b)

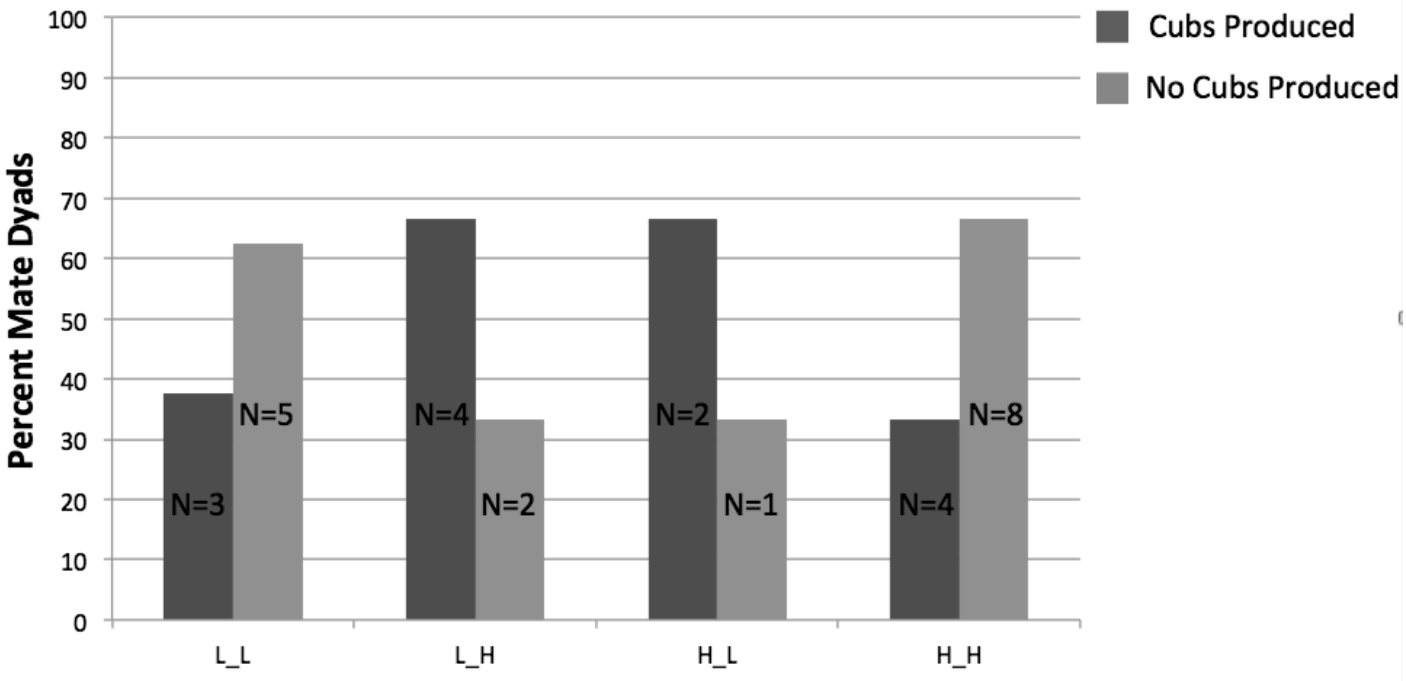

Mate Dyad Stereotypical Component Compatibility Group

Figure 4.4. The percent of mate dyads resulting in a) successful intromission and b) cub production for mate pairings of females and males that were both ranked low on stereotypical-excitable component scores $\left(L_{-} L\right)$, of females that were ranked low and males that were ranked high $\left(L_{-} H\right)$, of females that were ranked high and males that were ranked low $\left(H_{-} L\right)$, and of females and males that were both ranked high $\left(\mathrm{H}_{-} \mathrm{H}\right)$. $\mathrm{N}$ represents the number of mate dyads. * indicates $p \leq 0.05$ 


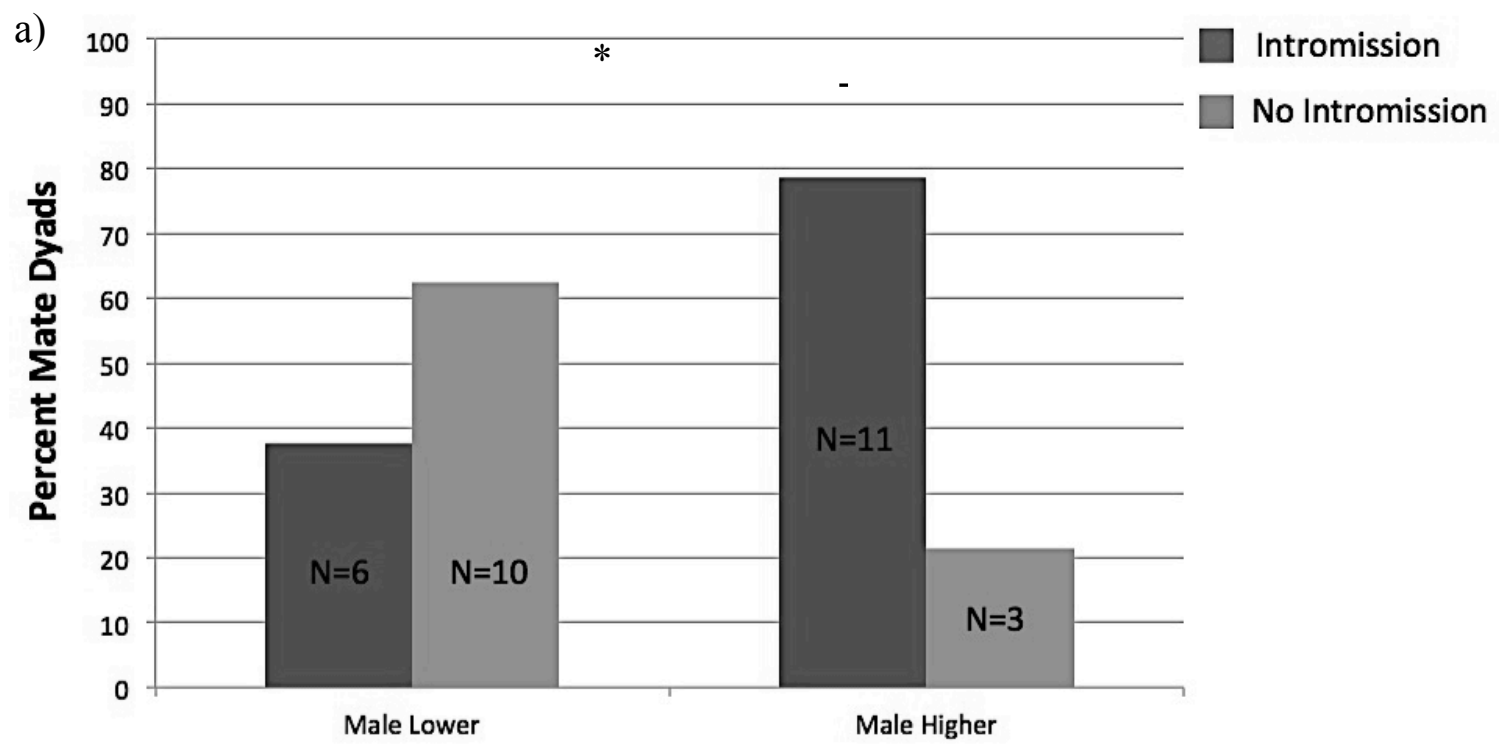

b)

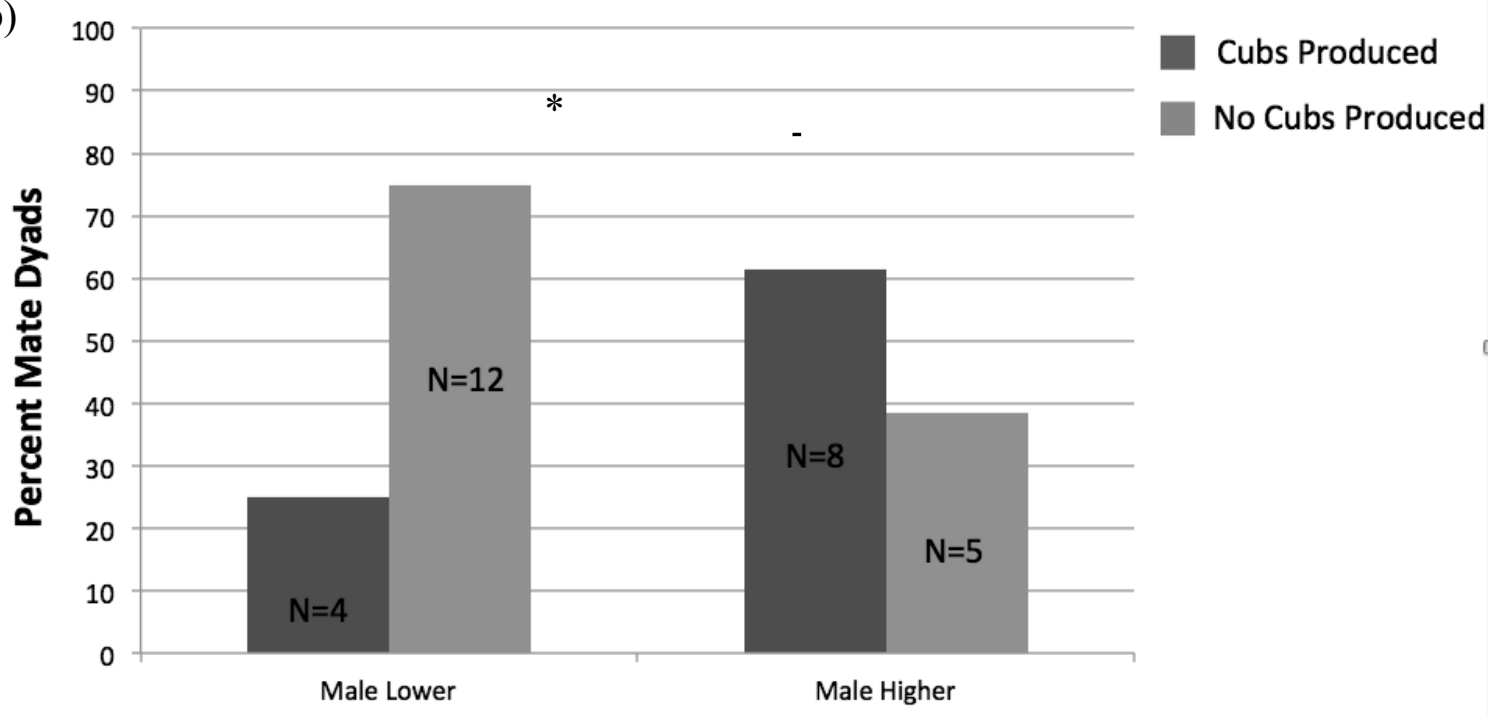

Male Aggressive Component Score Relative to Female

Figure 4.5. The percent of mate dyads resulting in a) successful intromission and b) cub production for mate pairings composed of females mated to males ranked lower than them on aggressive component scores and females mated to males ranked higher than them on aggressive component scores. $\mathrm{N}$ represents the number of mate dyads. *indicates $p \leq 0.05$ 


\section{CHAPTER 5: CONCLUSIONS}

Captive breeding provides an opportunity to augment natural populations where declines in nature may have impaired a species' capacity to recover from environmental stresses, as well as to re-establish extirpated populations (Soulé et al. 1986). To maintain healthy genetic variation, captive breeding programs require that the majority of the reproductively mature adults in the population reproduce successfully. However, in practice many captive-bred individuals fail to reproduce, even when presumably fully reproductive and healthy. Historically, captive breeding programs have focused primarily on pairing animals for breeding based on their pedigree and the future genetic health of the population (Snyder et al. 2002), while largely ignoring the potential importance of behavioral compatibility such as mate preference and personality (Swaisgood \& Schulte 2010). Such methods have been largely unsuccessful at maintaining and/or growing populations of rare mammals (AZA Sustainable Zoo \& Aquarium Populations Report 2013). At present, many questions about how personality and mate preference operate on reproductive performance in the wide variety of species represented in zoos remain unanswered.

Giant pandas are notoriously poor breeders in captivity (Swaisgood et al. 2003b) but in China methodology for their breeding provides an ideal research system to rigorously test hypotheses and samples sizes are large. Because of our collaboration with the CCRCGP we were able to address specific questions regarding the role of mate preference and personality in reproductive performance:

1) Can incorporation of mate preference in breeding assignments increase reproductive performance in managed programs? 
2) Can incorporation of personality traits in assignment of breeding pairs increase reproductive performance?

Through dichotomous choice tests and carefully tracking intromission success and cub production we were able to investigate the effects of mate preference and personality on these reproductive performance measurements. We found that mate preference, individual personality, and certain personality combinations within a mate pair can all increase reproductive performance in the captive giant panda.

\section{Mate preference}

We found that mate preference had the highest correlation with intromission success and cub production out of all the factors we investigated that could affect reproductive performance. Pairing both male and female giant pandas with a preferred partner increased the probability of intromission success and of producing a cub. There was also an increase in intromission success and cub production between mate dyads where there was mutual mate preference (preferred-preferred pairings) versus dyads that did not prefer each other (nonpreferred-nonpreferred) pairings. These findings are in agreement with studies on other species where mate preference increases reproductive performance measurements and offspring fitness (Drickamer et al. 2000a; Drickamer et al. 2003; Gowaty et al. 2003; Martin \& Shepherdson 2012). Our research would indicate that management-induced constraints that inhibit or prevent female and male giant pandas from mating with preferred mates will result in reduced reproductive performance. Mate choice is thought to be an important source of evolutionary change (Drickamer et al. 2000a); hence, it may affect both physiological and morphological traits in progeny as well as the dynamics of social behavior. Future studies should focus on 
applying our findings to the giant panda breeding program and collecting data on whether the program success rate increases. In addition, preference and ways to modify preference should be investigated further. The following questions arose as we were analyzing data: What makes a male or female preferred? Can mate preference be detected via physiological cues? Can these traits be manipulated to increase the reproductive performance of genetically valuable individuals? Are the traits linked with preference (or preference itself) genetically passed on through generations (i.e. do preferred males produce sons and daughters that are chosen as preferred as well)? If we provide more than two choices of mates does reproductive performance increase further? Does a female's mate preference change as they enter estrus? Can we detect preference for opposite sex conspecifics outside of breeding season? Do individuals show stronger preference for specific opposite sex conspecifics as the fertile period approaches or does their preference become more flexible? Do males prefer females in estrus over nonestrous females? Do males prefer females in peak estrus versus females in earlier stages? Does male-male competition around a female increase a male's preference for that female and motivation to breed? All of these questions could potentially impact how and when breeding managers perform pre-mating and mating introductions in giant pandas. To expand my observations to captive breeding populations of endangered species in general, a multi-institutional survey could be conducted using the American Zoo Association definition for sustainable populations on whether sustainably versus nonsustainably captive bred species have free mate choice, limited mate choice, or no mate choice.

Familiarity also increased reproductive performance in a mate dyad. Successfully 
producing a cub in the past with a potential mate increased intromission success in both male and female giant pandas and increased cub production for female giant pandas. This result is consistent with findings in other species that mate familiarity increases mating success and production of offspring (Fisher et al. 2003b; Martin \& Shepherdson 2012; Roberts \& Gosling 2004b). Familiarity measurements in the year prior to breeding and directly leading up to breeding did not have an effect on the reproductive performance of a mate pairing. This would suggest that intensive movement of giant pandas prior to breeding is not necessary for increased reproductive performance and managers may be able to focus less intensely on moving pandas during the breeding season. However, more controlled studies on familiarity should be conducted through experimentally manipulating the amount of time potential mate pairs spend next to each other in the year prior to breeding before breeding management changes are implemented. We suggest setting up an experiment where some females are housed next to potential mates for the year prior to breeding (long-term familiar pairs) and some females are housed next to males solely in the time period directly prior to breeding (nonfamiliar pairs). In addition, the following questions arose as we were analyzing data: When does familiarity arise? Does it take successful cub production or years of constant contact? What signals are used for establishing familiarity? Does chemical communication through scent swapping establish familiarity? What are the movement of wild male and female pandas prior to breeding season and how does this affect their reproductive performance? Should captive settings replicate wild settings in terms of social housing? Is familiarity of conspecifics important for individual welfare, and does disruption of these social relationships cause an increase in stress? 
We found that females mated to larger, heavier males had more intromissions and produced more cubs than females mated to smaller males. In follow-up analyses (Table A.3 in Appendix A), we found that females preferred older males but that male age was highly correlated with male mass in our data set. We must infer a lot in order to make sense of these findings, but we suggest older males may weigh more than younger males and the correlation between male mass and intromission success in our current GLMM may actually be indicating female preference. A more thorough statistical investigation into male mass and preference needs to be conducted before a solid conclusion can be reached. The finding that male mass increases reproductive performance would be consistent with recent experiments on wild pandas that suggests dominant giant panda males that secure breeding rights to females tend to be the largest male present during mating aggregations (Nie et al. 2012a). Male mass has been shown to be important for reproductive performance in many other species (Bisazza \& Marconato 1988; Harari et al. 1999; Hastings 1988; Hoelzer 1990; Howard et al. 1998; Poole 1989).

Surprisingly, for male based intromission success, male mass was not a significant factor. However, mass was correlated with male cub production. We suggest that this finding can be explained via female preference discussed above. It makes sense that females can distinguish between heavier and lighter males and that this would affect intromission success from a choosing female's perspective (i.e. females can readily assess male size in pre-mate introductions; Charlton et al. 2009). However, in the current system where there is no male-male competition, when intromission success is evaluated from the male perspective, small and large males should invest the same amount of energy in courtship behaviors and achieving intromission. Further, ejaculate 
characteristics for larger males could indicate that there may be post-copulatory mechanisms that could confer fitness benefits on males and would explain the cub production success without corresponding intromission success. Clearly the interaction of male mass on reproductive performance needs to be investigated further. Controlling dichotomous mate choice tests for male mass by presenting males of relatively similar mass or highly mismatching the mass in males (one very large and one very small) would help elucidate whether preference or mass is having more of an impact on reproductive performance. In addition, collecting semen samples during the breeding season for large and small males that are given opportunities to mate and correlating semen quality measurements with reproductive performance measurements would further inform the hypothesis that increased male mass increases ejaculate quality (in both ejaculate volume and sperm physiology). The following questions arose surrounding male mass and malemale competition as we were conducting our analyses: in light of wild studies, would larger males "win" competitions in captivity? Is there a clear "winner/loser" in malemale competition? Can we detect this through male-male pre-mating introductions (male-male competition) across mesh barriers? Would we see elevated levels of testosterone and cortisol in "winners" after the male-male pre-mating introductions? Would female preference change if she were allowed to witness male-male competitions? Would male sexual motivation change after having male-male competition opportunities? Would reproductive performance increase if males were given male-male competition? 


\section{Personality}

We investigated the effect of personality on the long-term reproductive performance of giant pandas. The four major personality characteristics determined from PCA were aggressive, playful-clever, fearful, and stereotypical-excitable. We found that both male and female fearful giant pandas had lower average yearly mating behaviors, low long-term natural mating success, and low long-term cub production. Additionally, aggressive female giant pandas had increased long-term natural mating success but decreased long-term cub production. Males did not show these additional relationships between aggressive personalities and reproductive performance. Fearfulness has been linked with decreased fitness in both wild and captive studies and across species, including giant pandas (Ariyomo et al. 2013; Powell et al. 2008; Réale \& Festa-Bianchet 2003; Réale et al. 2000; Smith \& Blumstein 2008). Our findings suggest that husbandry practices should consider personality traits of an individual to increase reproductive performance in the captive population. However, many questions remain to be answered about the relationship of personality to reproductive performance: are personality traits genetically determined? Can they be inherited from generation to generation or are they learned? If they can be inherited are they under selection pressures in captivity and is the population becoming more domesticated? Can altering an individual's personality (i.e. working with fearful animals to make them more bold) increase the reproductive performance of that individual? Does altering mating environments to accommodate personality traits (i.e. quieter pens with less human interaction for fearful individuals) increase reproductive performance of these animals? How may these personality types be affecting the survivorship of reintroduced giant pandas? Answering these questions 
could have important implications for the captive breeding program. Specifically, investigating domestication effects in giant pandas may be highly important for the success of the reintroduction programs that are currently taking place and to further guide future breeding efforts.

We further investigated the effects of personality on the reproductive performance of a specific mate dyad. Our results suggest that giant pandas appear to be mating assortatively on aggressive, fearful, and stereotypical-excitable personality traits. For aggression and stereotypical-excitable personality traits, mate pairs that were dissimilar in personality traits had higher intromission success and cub production. We found pairs where males were ranked high on these traits and females were ranked low (L_H dyads) had the highest reproductive performance. Aggression is cited as important for establishing dominance (Ellis 1995) in other species which confers reproductive benefits (Blanchard et al. 1984; DeVore 1965; Dewsbury 1984; Fleming \& Gross 1993; Fox 1983). Both male and female giant pandas needed to be ranked 'low' on fearfulness to have higher intromission success but mate dyad ranking on 'fearfulness' did not effect cub production. Our findings on 'fearfulness' are consistent with other captive studies on reproductive performance and personality (Powell et al. 2008; Wielebnowski 1999). To our knowledge, this is the first study that indicates stereotypical-excitable personality relative to a mating partner may increase reproductive performance. Our results suggest that integrating genetic information with behavioral measures of personality, specifically in relationship to the individual pandas being paired for mating, may also increase the reproductive output of the giant panda captive-breeding program. As referred to earlier, more research is needed on the effects of personality on reproductive performance to 
fully determine possible consequences. We had the following questions as we wrote up our results: Is personality stable in the breeding and non-breeding seasons? Does human presence affect behaviors of the different personality types during mate introductions? Do individual humans alter the behavioral/personality profiles of giant pandas and is this reflected on those keeper's personality questionnaires? Can we alter the reproductive performance of individuals through pairing them with certain personality types? Do stereotypical animals have higher or lower reproductive performance in captive settings? How does stereotypical behavior effect mate preference? Do stereotypical animals have higher or lower cortisol and testosterone measurements? How is this linked to environmental enrichment in adulthood and during infancy? Research has only recently started to investigate the role of stereotypical behavior on reproductive performance (Díez-León et al. 2013; Jones et al. 2010) and many questions remain unanswered. Clearly, research into personality could produce important information for the success of captive breeding programs and warrants further investigation.

\section{Final Conclusions}

In captive breeding programs, mates are traditionally selected solely on the basis of genetic parameters to minimize loss of genetic diversity and inbreeding coefficients. Our results suggest that integrating genetic with behavioral measures of preference and personality would increase the reproductive output of the giant panda captive-breeding program. These findings could be employed by breeding managers to increase mate compatibility, decrease stress, and enhance welfare and breeding success. Clearly, we must work within the confines of the genetic recommendations to increase reproductive performance in endangered species in order to create self-sustaining captive populations 
that, could in turn, be used as a reservoir for individuals being reintroduced back into the wild. However, with the knowledge gained from this study we will be able to suggest methods for improving the reproductive performance of genetically valuable animals by conducting assessment of mate acceptability before actual animal transfer and designing strategies of intra-institutional management that optimize reproductive performance. We suggest that captive breeding facilities for other endangered species investigate both mate preference and personality to improve the captive breeding methods currently utilized by both zoological and private breeding facilities. For the plethora of species currently endangered or declining, captivity may serve as the only fail-safe for their extinction. Considering the plight of these animals, research into increasing the success of captive breeding programs merits intensive and immediate attention. It seems clear that conservation breeding programs in zoos and breeding centers have much to gain from a more integrated approach, addressing both the importance of genetic management and behavioral analyses. Ultimately, close partnerships between researchers and breeding managers that maintain large populations of captive bred endangered species will ensure that lessons learned from research are rapidly incorporated into management strategies, as adaptive management programs. 


\section{LITERATURE CITED}

Aitken-Palmer, C., R. Hou, C. Burrell, Z. Zhang, C. Wang, R. Spindler, D. E. Wildt, M. A. Ottinger, and J. Howard. 2012. Protracted Reproductive Seasonality in the Male Giant Panda (Ailuropoda melanoleuca) Reflected by Patterns in Androgen Profiles, Ejaculate Characteristics, and Selected Behaviors 1. Biology of reproduction 86.

Anderson, W., K. Yong-Kyu, and P. Gowaty. 2007. Experimental constraints on mate preferences in Drosophila pseudoobscura decrease offspring viability and fitness of mated pairs. Proceedings of the National Academy of Sciences of the United States of America 104:4484-4488.

Aquariums., A. A. o. Z. a. 2013. Sustainable Zoo \& Aquarium Populations Report. AZA, Silver Spring, MD

Ariyomo, T. O., M. Carter, and P. J. Watt. 2013. Heritability of boldness and aggressiveness in the zebrafish. Behavior genetics 43:161-167.

Asa, C., K. Traylor-Holzer, and R. Lacy. 2011. Can conservation-breeding programmes be improved by incorporating mate choice? International Zoo Yearbook 45:203212.

Baker, A. 2007. Animal ambassadors: an analysis of the effectiveness and conservation impact of ex situ breeding efforts. Pages 139-154 in A. Zimmermann, M. Hatchwell, L. A. Dickie, and C. West, editors. Zoos in the 21st Century: Catalysts for Conservation? Cambridge University Press, Cambridge.

Bisazza, A., and A. Marconato. 1988. Female mate choice, male-male competition and parental care in the river bullhead, $<\mathrm{i}>$ Cottus gobio $</ \mathrm{i}>$ L.(Pisces, Cottidae). Animal Behaviour 36:1352-1360.

Blanchard, D. C., C. Fukunaga-Stinson, L. K. Takahashi, K. J. Flannelly, and R. J. Blanchard. 1984. Dominance and aggression in social groups of male and female rats. Behavioural processes 9:31-48.

Both, C., N. J. Dingemanse, P. J. Drent, and J. M. Tinbergen. 2005. Pairs of extreme avian personalities have highest reproductive success. Journal of Animal Ecology 74:667-674.

Boughman, J. W. 2002. How sensory drive can promote speciation. Trends in Ecology \& Evolution 17:571-577.

Budaev, S. V. 1999. Sex differences in the Big Five personality factors: Testing an evolutionary hypothesis. Personality and individual differences 26:801-813.

Campbell, P., T. Pottinger, and J. Sumpter. 1994. Preliminary evidence that chronic confinement stress reduces the quality of gametes produced by brown and rainbow trout. Aquaculture 120:151-169.

Carlstead, K., J. Fraser, C. Bennett, and D. G. Kleiman. 1999a. Black rhinoceros (Diceros bicornis) in US zoos: II. Behavior, breeding success, and mortality in relation to housing facilities. Zoo Biology 18:35-52.

Carlstead, K., J. Mellen, and D. G. Kleiman. 1999b. Black rhinoceros (Diceros bicornis) in US zoos: I. Individual behavior profiles and their relationship to breeding success. Zoo Biology 18:17-34. 
Carlstead, K., and D. Shepherdson. 1994. Effects of Environmental Enrichment on Reproduction. Zoo Biology 13:447-458.

Carson, H. L. 2003. Mate choice theory and the mode of selection in sexual populations. Proceedings of the Royal Society B: Biological Sciences 100:6584-6587.

Charlton, B., Y. Huang, and R. R. Swaisgood. 2009. Vocal discrimination of potential mates by female giant pandas (Ailuropoda melanoleuca). Biology Letters 5:597599.

Cheetham, S. A., M. D. Thom, R. J. Beynon, and J. L. Hurst. 2008. The effect of familiarity on mate choice. Pages 271-280. Chemical Signals in Vertebrates 11. Springer.

Choudhury, S., J. M. Black, and M. Owen. 1996. Body size, fitness and compatibility in Barnacle Geese Branta leucopsis. Ibis 138:700-709.

Christie, M. R., M. L. Marine, R. A. French, and M. S. Blouin. 2012. Genetic adaptations to captivity can occur ina single generation. 109:238-242.

Cloninger, C. R., D. M. Svrakic, and T. R. Przybeck. 1993. A psychobiological model of temperament and character. Archives of general psychiatry 50:975-990.

Cole, E. F., and J. L. Quinn. 2014. Shy birds play it safe: personality in captivity predicts risk.

Conrad, J. L., K. L. Weinersmith, T. Brodin, J. Saltz, and A. Sih. 2011. Behavioural syndromes in fishes: a review with implications for ecology and fisheries management. Journal of Fish Biology 78:395-435.

Crawley, M. J. 2005. Statistics: an introduction using R, 2005. Wiley, ISBN 0 470:1.

Cyr, N. E., and L. Michael Romero. 2007. Chronic stress in free-living European starlings reduces corticosterone concentrations and reproductive success. General and comparative endocrinology 151:82-89.

Darwin, C. 1859. On the Origin of Species. 1. London: Murray.

Darwin, C. 1871. On the Origin of Species by Means of Natural Selection (London, 1859). The Descent of Man.

DeVore, I. 1965. Male dominance and mating behavior in baboons. Sex and behavior 266:289.

Dewsbury, D. A. 1984. Aggression, copulation, and differential reproduction of deer mice (Peromyscus maniculatus) in a semi-natural enclosure. Behaviour:1-23.

Díez-León, M., J. Bowman, S. Bursian, H. Filion, D. Galicia, J. Kanefsky, A. Napolitano, R. Palme, A. Schulte-Hostedde, and K. Scribner. 2013. Environmentally Enriched Male Mink Gain More Copulations than Stereotypic, Barren-Reared Competitors. PloS one 8:e80494.

Dingemanse, N. J., C. Both, P. J. Drent, and J. M. Tinbergen. 2004. Fitness consequences of avian personalities in a fluctuating environment. Proceedings of the Royal

Society of London, Series B: Biological Sciences 271:847-852.

Dingemanse, N. J., C. Both, P. J. Drent, K. Van Oers, and A. J. Van Noordwijk. 2002. Repeatability and heritability of exploratory behaviour in great tits from the wild. Animal Behaviour 64:929-938.

Dingemanse, N. J., and D. Réale. 2005. Natural selection and animal personality. Behaviour 142:9-10. 
Doutrelant, C., and P. K. McGregor. 2000. EAVESDROPPINGAND MATE CHOICE IN FEMALE FIGHTING FISH. Behaviour 137:1655-1669.

Drickamer, L., P. Gowaty, and C. Holmes. 2000a. Free female mate choice in house mice affects reproductive success and offspring viability and performance. Animal Behavior 59.

Drickamer, L., P. Gowaty, and C. Holmes. 2000b. Free female mate choice in house mice affects reproductive success and offspring viability and performance. Animal Behavior 59:371-378.

Drickamer, L., P. Gowaty, and D. Wagner. 2003. Free mutual mate preferences in house mice affect reproductive success and offspring performance. Animal Behavior 65:105-114.

EC. 2001. The welfare of animals kept for fur production. Commision of the European Communities, Brussels, Belgium.

Eizaguirre, C., S. E. Yeates, T. L. Lenz, M. Kalbe, and M. Milinski. 2009. MHC-based mate choice combines good genes and maintenance of MHC polymorphism. Molecular ecology 18:3316-3329.

Ellis, L. 1995. Dominance and reproductive success among nonhuman animals: a crossspecies comparison. Ethology and Sociobiology 16:257-333.

Ellis, S., R. J. Snyder, G. Zhang, R. Wei, W. Zhong, M. Lam, and R. Sims. 2006. Life histories and behavioral traits as predictor of breeding success. Pages 87-100 in D. E. Wildt, Z. Anju, Z. Hemin, D. Janssen, L. , and S. Ellis, editors. Giant Pandas: Biology, Veterinary Medicine and Management. Cambridge University Press, Cambridge.

Elsbeth McPhee, M. 2004. Generations in captivity increases behavioral variance: considerations for captive breeding and reintroduction programs. Biological Conservation 115:71-77.

Fisher, H. S., R. Swaisgood, and H. Fitch-Snyder. 2003a. Odor familiarity and female preferences for males in a threatened primate, the pygmy loris Nycticebus pygmaeus: applications for genetic management of small populations. Naturwissenschaften 90:509-512.

Fisher, H. S., R. Swaisgood, and H. Fitch-Snyder. 2003b. Odor familiarity and female preferences for males in a threatened primate, the pygmy loris Nycticebus pygmaeus: applications for genetic management of small populations. Naturwissenschaften 90:509-512.

Fisher, R. A. 1915. The evolution of sexual preference. The Eugenics Review 7:184.

Flannery, K. V., P. J. UCKO, and G. DIMBLEBY. 1969. Origins and ecological effects of early domestication in Iran and the Near East. The domestication and exploitation of plants and animals.:73-100.

Fleming, I. A., and M. R. Gross. 1993. Breeding success of hatchery and wild coho salmon (Oncorhynchus kisutch) in competition. Ecological Applications:230-245.

Fox, S. 1983. Fitness, home-range quality, and aggression in Uta stansburiana. Lizard ecology: Studies of a model organism:149-168.

Gabriel, P. O., and J. M. Black. 2012. Behavioural syndromes, partner compatibility and reproductive performance in Steller's jays. Ethology 118:76-86. 
Galef, J., G. Bennett, and D. J. WHITE. 1998. Mate-choice copying in Japanese quail,< $\mathrm{i}>$ Coturnix coturnix japonica $</ \mathrm{i}>$. Animal Behaviour 55:545-552.

Garamszegi, L. Z. 2006. Comparing effect sizes across variables: generalization without the need for Bonferroni correction. Behavioral Ecology 17:682-687.

Garner, J. P., and G. J. Mason. 2002. Evidence for a relationship between cage stereotypies and behavioural disinhibition in laboratory rodents. Behavioural Brain Research 136:83-92.

Geber, W. F. 1966. Developmental effects of chronic maternal audiovisual stress on the rat fetus. Journal of embryology and experimental morphology 16:1-16.

Geggel, L. 2014. Triplets! Giant Panda Gives Birth to 'Rare' Trio in China. TechMedia Network, LiveScience

Gilpin, M. E., and M. E. Soule. 1986. Minimum viable populations: Processes of species extinction. Pages 19-34 in M. E. Soule, editor. Conservation Biology: The Science of Scarcity and Diversity. Sinauer, Sunderland, Massachutes.

Gold, K. C., and T. L. Maple. 1994. Personality assessment in the gorilla and its utility as a management tool. Zoo Biology 13:509-522.

Gosling, L. M., and W. J. Sutherland 2000. Behaviour and conservation. Cambridge University Press.

Gosling, S. D. 1998. Personality dimensions in spotted hyenas $(<\mathrm{em}>$ Crocuta crocuta $</$ em $>$ ). Journal of Comparative Psychology 112:107.

Gosling, S. D. 2001. From mice to men: what can we learn about personality from animal research? Psychological bulletin 127:45.

Gowaty, P. A., L. C. Drickamer, and S. Schmid-Holmes. 2003. Male house mice produce fewer offspring with lower viability and poorer performance when mated with females they do not prefer. Animal Behaviour 65:95-103.

Hamilton, W. D., and M. Zuk. 1982. Heritable true fitness and bright birds: a role for parasites? Science 218:384-387.

Harari, A. R., A. M. Handler, and P. J. Landolt. 1999. Size-assortative mating, male choice and female choice in the curculionid beetle $<\mathrm{i}>$ Diaprepes abbreviatus $</ \mathrm{i}>$. Animal Behaviour 58:1191-1200.

Hastings, P. A. 1988. Female choice and male reproductive success in the angel blenny, < $\mathrm{i}>$ Coralliozetus angelica $</ \mathrm{i}>$ (Teleostei: Chaenopsidae). Animal Behaviour 36:115-124.

Hausberger, M., C. Bruderer, N. Le Scolan, and J.-S. Pierre. 2004. Interplay Between Environmental and Genetic Factors in Temperament/Personality Traits in Horses $<$ em $>$ (Equus caballus $)</$ em $>$. Journal of Comparative Psychology 118:434.

Hoelzer, G. A. 1990. Male-male competition and female choice in the Cortez damselfish, $<\mathrm{i}>$ Stegastes rectifraenum $</ \mathrm{i}>$. Animal Behaviour 40:339-349.

Höglund, J., R. V. Alatalo, and A. Lundberg. 1990. Copying the mate choice of others? Observations on female black grouse. Behaviour:221-231.

Hohoff, C., K. Franzen, and N. Sachser. 2003. Female choice in a promiscuous wild guinea pig, the yellow-toother cavy (Galea musteloides). Behavioral Ecology and Sociobiology 53:341-349.

Howard, J., Z. Zhang, D. Li, Y. Huang, R. Hou, G. Li, M. Zhang, Z. Ye, J. Zhang, and S. Huang. 2006. Male reproductive biology in giant pandas in breeding programmes 
in China. Giant pandas: biology, veterinary medicine and management.

Cambridge University Press, Cambridge, UK:159-197.

Howard, R., R. Martens, S. Innis, J. Drnevich, and J. Hale. 1998. Mate choice and mate competition influence male body size in Japanese medaka. Animal Behaviour 55:1151-1163.

Jocklin, V., M. McGue, and D. T. Lykken. 1996. Personality and divorce: a genetic analysis. Journal of personality and social psychology 71:288.

Johnstone, R. A., J. D. Reynolds, and J. C. Deutsch. 1996. Mutual mate choice and sex differences in choosiness. Evolution:1382-1391.

Jones, C., W. Heck, R. Lewis, Y. Mungroo, G. Slade, and T. Cade. 1995. The restoration of the Mauritius Kestrel Falco punctatus population. Ibis 137 (Suppl.):S173S180.

Jones, M. A., M. van Lierop, G. Mason, and N. Pillay. 2010. Increased reproductive output in stereotypic captive $<\mathrm{i}>$ Rhabdomys $</ \mathrm{i}>$ females: Potential implications for captive breeding. Applied Animal Behaviour Science 123:63-69.

Jones, R. B., A. D. Mills, and J.-M. Faure. 1991. Genetic and experiential manipulation of fear-related behavior in Japanese quail chicks $(<\mathrm{em}>$ Coturnix coturnix japonica $</$ em $>$ ). Journal of Comparative Psychology 105:15.

Kleiman, D. G. 1983. Ethology and Reproduction of Captive Giant Pandas (Ailuropoda melanoleuca). Zeitschrift für Tierpsychologie 62:1-46.

Klint, T., and M. Enquist. 1981. Pair formation and reproductive output in domestic pigeons. Behavioral Process 6:57-62.

Kokko, H., and R. Brooks. 2003. Sexy to die for? Sexual selection and the risk of extinction. Annals of Zoology Fenn 40:207-219.

Kraaijeveld-Smit, F. J., R. A. Griffiths, R. D. Moore, and T. J. Beebee. 2006. Captive breeding and the fitness of reintroduced species: a test of the responses to predators in a threatened amphibian. Journal of Applied Ecology 43:360-365.

Le Boeuf, B. J. 1974. Male-male competition and reproductive success in elephant seals. American Zoologist 14:163-176.

Lees, C. M., and J. Wilcken. 2009a. Sustaining the Ark: the challenges faced by zoos in maintaining viable populations. International Zoo Yearbook 43:6-18.

Lees, C. M., and J. Wilcken. 2009b. Sustaining the Ark: the challenges faced by zoos in maintaining viable populations. . International Zoo Yearbook 43:6-18.

Lenkoski, A., T. S. Eicher, and A. E. Raftery. 2014. Two-stage Bayesian model averaging in endogenous variable models. Econometric reviews 33:122-151.

Lewis, O. T., and C. D. Thomas. 2001. Adaptations to captivity in the butterfly Pieris brassicae (L.) and the implications for ex situ conservation. Journal of Insect Conservation 5:55-63.

Lindburg, D. G., X. Huang, and S. Huang. 1998. Reproductive performance of giant panda males in Chinese zoos in A. Zhang, and G. He, editors. International Symposium on the Protection of the Giant Panda (Ailuropoda melanoleuca). Sichuan Publishing House of Science and Technology, Chengdu, China.

Liu, D., Z. Wang, H. Tian, C. Yu, G. Zhang, R. Wei, and H. Zhang. 2003. Behavior of giant pandas (Ailuropoda melanoleuca) in captive conditions: Gender differences and enclosure effects. Zoo Biology 22:77-82. 
Liu, J., Y. Chen, L. Guo, B. Gu, H. Liu, A. Hou, X. Liu, L. Sun, and D. Liu. 2006. Stereotypic behavior and fecal cortisol level in captive giant pandas in relation to environmental enrichment. Zoo Biology 25:445-459.

Lu, Z., W. Pan, and J. Harkness. 1994. Mother-cub relationships in giant pandas in the Qinling Mountains, China, with comment on rescuing abandoned cubs. Zoo Biology 13:567-568.

Marieke Cassia, G., and P. David. 2012. Personality assessment in snow leopards (Uncia uncia). Zoo biology 31:151-165.

Martin, M. S., and D. J. Shepherdson. 2012. Role of Familiarity and Preference in Reproductive Success in Ex Situ Breeding Programs. Conservation Biology 26:649-656.

Marzluff, J. M., G. E. Woolfenden, J. Fitzpatrick, and R. Balda. 1996. Breeding partnerships of two New World jays. OXFORD ORNITHOLOGY SERIES 6:138-161.

Mason, G. 1991. Stereotypies and suffering. Behavioural Processes 25:103-115.

Mason, G. 2006. Stereotypic behaviour in captive animals: fundamentals and implications for welfare and beyond. Stereotypic Animal Behaviour: Fundamentals and Applications to Welfare:325-356.

Mason, G., and N. Latham. 2004. Can't stop, won't stop: is stereotypy a reliable animal welfare indicator? Animal Welfare.

McCullagh, P., and J. A. Nelder 1989. Generalized Linear Models, Second Edition. Taylor and Francis.

McDougall, P., D. Réale, D. Sol, and S. Reader. 2006. Wildlife conservation and animal temperament: causes and consequences of evolutionary change for captive, reintroduced, and wild populations. Animal Conservation 9:39-48.

McGeehan, L., X. Li, L. Jackintell, S. Huang, A. Wang, and N. M. Czekala. 2002. Hormonal and behavioral correlates of estrus in captive giant pandas. Zoo Biology 21:449-466.

Mendl, M., A. J. Zanella, and D. M. Broom. 1992. Physiological and reproductive correlates of behavioural strategies in female domestic pigs. Animal Behaviour 44:1107-1121.

Moller, A. P. 2003. Sexul selection and extinction: why sex matters and why asexual models are insufficient. Annals of Zoology Fenn 40:221-230.

Møller, A. P., and S. Legendre. 2001. Allee effect, sexual selection and demographic stochasticity. Oikos 92:27-34.

Momozawa, Y., T. Ono, F. Sato, T. Kikusui, Y. Takeuchi, Y. Mori, and R. Kusunose. 2003. Assessment of equine temperament by a questionnaire survey to caretakers and evaluation of its reliability by simultaneous behavior test. Applied Animal Behaviour Science 84:127-138.

Monfort, S., K. Dahl, N. Czekala, L. Stevens, M. Bush, and D. Wildt. 1989. Monitoring ovarian function and pregnancy in the giant panda (Ailuropoda melanoleuca) by evaluating urinary bioactive FSH and steroid metabolites. Journal of Reproduction and Fertility 85:203-212.

Myers, R. E. 1975. Maternal psychological stress and fetal asphyxia: a study in the monkey. Am J Obstet Gynecol 122:47-59. 
Nakagawa, S. 2004. A farewell to Bonferroni: the problems of low statistical power and publication bias. Behavioral Ecology 15:1044-1045.

Nie, Y., R. R. Swaisgood, Z. Zhang, Y. Hu, Y. Ma, and F. Wei. 2012a. Giant panda scent-marking strategies in the wild: role of season, sex and marking surface. Animal Behaviour 84:39-44.

Nie, Y., R. R. Swaisgood, Z. Zhang, X. Liu, and F. Wei. 2012b. Reproductive competition and fecal testosterone in wild male giant pandas (Ailuropoda melanoleuca). Behavioral Ecology and Sociobiology 66:721-730.

Nie, Y.-G., Z.-J. Zhang, R. R. Swaisgood, and F.-W. Wei. 2012c. Effects of season and social interaction on fecal testosterone metabolites in wild male giant pandas: implications for energetics and mating strategies. European Journal of Wildlife Research 58:235-241.

Nowicki, S., and K. B. Armitage. 1979. Behavior of Juvenile Yellow-bellied Marmots: Play and Social Integration. Zeitschrift für Tierpsychologie 51:85-105.

Peng, J., Z. Jiang, W. Liu, S. Huang, J. Zhang, and W. Wang. 2001. Growth and development of giant panda (Ailuropoda melanoleuca) cubs at Beijing Zoo. Journal of Zoology 254:261-266.

Peng, J., Z. Jiang, G. Qin, Q. Huang, Y. Li, Z. Jiao, F. Zhang, H. Fang, J. Zhang, and Y. Lu. 2009. Mate choice in giant panda (Ailuropoda melanoleuca). THE ROYAL BELGIAN SOCIETY FOR ZOOLOGY 139:87-92.

Poole, J. H. 1989. Mate guarding, reproductive success and female choice in African elephants. Animal Behaviour 37:842-849.

Powell, D., L. Hong, K. Carlstead, D. Kleiman, H. Zhang, G. Zhang, Z. Zhang, J. Yu, J. Zhang, and Y. Lu. 2008. Relationships between temperament, husbandry, management, and socio-sexual behavior in captive male and female giant pandas (Ailuropoda melanoleuca). Acta Zool Sinica 54:169-175.

Powell, D. M., and J. T. Svoke. 2008. Novel environmental enrichment may provide a tool for rapid assessment of animal personality: a case study with giant pandas (Ailuropoda melanoleuca). Journal of Applied Animal Welfare Science 11:301318.

Price, T., D. Schluter, and N. E. Heckman. 1993. Sexual selection when the female directly benefits. Biological Journal of the Linnean Society 48:187-211.

Quader, S. 2005. Mate choice and its implications for conservation and management Current Science 89:1220-1227.

Rabin, L. 2003. Maintaining behavioural diversity in captivity for conservation: natural behaviour management. Animal Welfare 12:85-94.

Réale, D., and M. Festa-Bianchet. 2003. Predator-induced natural selection on temperament in bighorn ewes. Animal behaviour 65:463-470.

Réale, D., B. Y. Gallant, M. Leblanc, and M. Festa-Bianchet. 2000. Consistency of temperament in bighorn ewes and correlates with behaviour and life history. Animal Behaviour 60:589-597.

Réale, D., S. M. Reader, D. Sol, P. T. McDougall, and N. J. Dingemanse. 2007. Integrating animal temperament within ecology and evolution. Biological reviews 82:291-318. 
Reby, D., M. Hewison, M. Izquierdo, and D. Pépin. 2001. Red Deer (Cervus elaphus) Hinds Discriminate Between the Roars of Their Current Harem-Holder Stag and Those of Neighbouring Stags. Ethology 107:951-959.

Rich, T. J., and J. L. Hurst. 1998. Scent marks as reliable signals of the competitive ability of mates. Animal Behaviour 56:727-735.

Rindos, D., and R. C. Dunnell 1984. The origins of agriculture: an evolutionary perspective. Academic Press New York.

Roberts, S. C., and L. M. Gosling. 2004a. Manipulation of olfactory signaling and mate choice for conservation breeding: a case study of harvest mice. Conservation Biology 18:548-556.

Roberts, S. C., and L. M. Gosling. 2004b. Manipulation of Olfactory Signaling and Mate Choice for Conservation Breeding: a Case Study of Harvest Mice. Conservation Biology 18:548-556.

Rushen, J., A. B. Lawrence, and E. C. Terlouw. 1993. The motivational basis of stereotypies.

Ryan, K. K., and J. Altmann. 2001. Selection for male choice based primarily on mate compatibility in the oldfield mouse, Peromyscus polionotus rhoadsi. Behavioral Ecology and Sociobiology 50:436-440.

Sandvik, M., G. Rosenqvist, and A. Berglund. 2000. Male and female mate choice affects offspring quality in a sex-role-reversed pipefish. Proceedings of the Royal Society B: Biological Sciences 267:2151-2155.

Schaller, G. B., H. Jinchu, P. Wenshi, and Z. Jing 1985. The Giant Pandas of Wolong. University Of Chicago Press, Chicago.

Schuett, W., S. R. Dall, and N. J. Royle. 2011. Pairs of zebra finches with similar 'personalities' make better parents. Animal Behaviour 81:609-618.

Shepherdson, D., K. Carlstead, and N. Wielebnowski. 2004. Cross-institutional assessment of stress responses in zoo animals using longitudinal monitoring of faecal corticoids and behaviour. ANIMAL WELFARE-POTTERS BAR THEN WHEATHAMPSTEAD- 13:S105-S114.

Shepherdson, D., K. D. Lewis, K. Carlstead, J. Bauman, and N. Perrin. 2013. Individual and environmental factors associated with stereotypic behavior and fecal glucocorticoid metabolite levels in zoo housed polar bears. Applied Animal Behaviour Science 147:268-277.

Sih, A., A. Bell, and J. C. Johnson. 2004. Behavioral syndromes: an ecological and evolutionary overview. Trends in Ecology \& Evolution 19:372-378.

Sinn, D., L. Apiolaza, and N. Moltschaniwskyj. 2006. Heritability and fitness-related consequences of squid personality traits. Journal of evolutionary biology 19:14371447.

Small, M. F. 1982. Reproductive failure in macaques. American Journal of Primatology 2:137-147.

Smith, B. R., and D. T. Blumstein. 2008. Fitness consequences of personality: a metaanalysis. Behavioral Ecology 19:448-455.

Snyder, N. F., S. R. Derrickson, S. R. Beissinger, J. W. Wiley, T. B. Smith, W. D. Toone, and B. Miller. 1996a. Limitations of captive breeding in endangered species recovery. Conservation Biology 10:338-348. 
Snyder, N. F. R., S. R. Derrickson, S. R. Beissinger, J. W. Wiley, T. B. Smith, W. D. Toone, and B. Miller. 1996b. Limitations of Captive Breeding in Endangered Species Recovery. Conservation Biology 10:338-348.

Snyder, N. F. R., S. R. Derrickson, S. R. Beissinger, J. W. Wiley, T. B. Smith, W. D. Toone, and B. Miller. 2002. Limitations of Captive Breeding in Endangered Species Recovery. Conservation Biology 10:338-348.

Snyder, R. J., A. J. Zhang, Z. H. Zhang, G. H. Li, Y. Z. Tian, X. M. Huang, L. Luo, M. A. Bloomsmith, D. L. Forthman, and T. L. Maple. 2003. Behavioral and developmental consequences of early rearing experience for captive giant pandas (Ailuropoda melanoleuca). Journal of Comparative Psychology 117:235.

Soulé, M., M. Gilpin, W. Conway, and T. Foose. 1986. The millenium ark: How long a voyage, how many staterooms, how many passengers? Zoo Biology 5:101-113.

Stockwell, C. A., and M. V. Ashley. 2004. Rapid adaptation and conservation. Conservation Biology 18:272-273.

Stockwell, C. A., A. P. Hendry, and M. T. Kinnison. 2003. Contemporary evolution meets conservation biology. Trends in Ecology \& Evolution 18:94-101.

Stoebel, D., and G. Moberg. 1982. Effect of adrenocorticotropin and cortisol on luteinizing hormone surge and estrous behavior of cows. Journal of dairy science 65:1016-1024.

Swaisgood, R. R., S. Ellis, D. L. Forthman, and D. J. Shepherdson. 2003a. Commentary: Improving well-being for captive giant pandas: Theoretical and practical issues. Zoo Biology 22:347-354.

Swaisgood, R. R., D. G. Lindburg, and X. Zhou. 1999. Giant pandas discriminate individual differences in conspecific scent. Animal Behaviour 57:1045-1053.

Swaisgood, R. R., D. G. Lindburg, X. Zhou, and M. A. Owen. 2000. The effects of sex, reproductive condition and context on discrimination of conspecific odours by giant pandas. Animal Behaviour 60:227-237.

Swaisgood, R. R., and M. A. Owen. 2007. Giant Panda Research \& Husbandry Information Packet. San Diego Zoo, San Diego, CA.

Swaisgood, R. R., M. A. Owen, N. M. Czekala, N. Mauroo, K. Hawk, and J. C. L. Tang. 2006. Evaluating stress and well-being in the giant panda: a system for monitoring in D. E. Wildt, editor. Giant pandas: biology, veternariy medicine and management. Cambridge University Press, Cambridge.

Swaisgood, R. R., and B. A. Schulte. 2010. Applying knowledge of mammalian social organization, mating systems, and communication to management in D. G. Kleiman, K. V. Thompson, and C. K. Baer, editors. Wild mammals in captivity : principles and techniques for zoo management. The University of Chicago Press, Chicago; London.

Swaisgood, R. R., A. M. White, X. Zhou, H. Zhang, G. Zhang, R. Wei, V. J. Hare, E. M. Tepper, and D. G. Lindburg. 2001. A quantitative assessment of the efficacy of an environmental enrichment programme for giant pandas. Animal Behaviour 61:447-457.

Swaisgood, R. R., X. Zhou, G. Zhang, D. G. Lindburg, and H. Zhang. 2003b. Application of behavioral knowledge to conservation in the giant panda. International journal of comparative psychology 16. 
Trut, L. 1999. Early Canid Domestication: The Farm-Fox Experiment Foxes bred for tamability in a 40-year experiment exhibit remarkable transformations that suggest an interplay between behavioral genetics and development. American Scientist 87:160-169.

Watters, J. V., and D. M. Powell. 2012. Measuring animal personality for use in population management in zoos: suggested methods and rationale. Zoo biology 31:1-12.

Wechsler, B. 1995. Coping and coping strategies: a behavioural view. Applied Animal Behaviour Science 43:123-134.

Wedekind, C., R. Muller, and H. Spicher. 2001. Potential genetic benefits of mate selection in whitefish. Journal of Evolutionary Biology 14:980-986.

Weiss, A., J. E. King, and R. M. Enns. 2002. Subjective well-being is heritable and genetically correlated with dominance in chimpanzees $(<\mathrm{em}>$ Pan troglodytes $</$ em $>$ ). Journal of Personality and Social Psychology 83:1141.

Weiss, A., J. E. King, and A. J. Figueredo. 2000. The heritability of personality factors in chimpanzees (Pan troglodytes). Behavior genetics 30:213-221.

White, A., R. Swaisgood, and H. Zhang. 2002. The highs and lows of chemical communication in giant pandas (\&lt;SMALL\&gt;Ailuropoda melanoleuca\&lt;/SMALL\&gt;): effect of scent deposition height on signal discrimination. Behavioral Ecology and Sociobiology 51:519-529.

Wielebnowski, N., and J. L. Brown. 1998. Behavioral correlates of physiological estrus in cheetahs. Zoo Biology 17:193-209.

Wielebnowski, N. C. 1999. Behavioral differences as predictors of breeding status in captive cheetahs. Zoo Biology 18:335-349.

Zajitschek, S. R., J. P. Evans, and R. Brooks. 2006. Independent effects of familiarity and mating preferences for ornamental traits on mating decisions in guppies. Behavioral Ecology 17:911-916.

Zhang, G., R. R. Swaisgood, and H. Zhang. 2004a. Evaluation of behavioral factors influencing reproductive success and failure in captive giant pandas. Zoo biology $23 \quad$ (1), p. 15. 23:15-31.

Zhang, G., R. R. Swaisgood, and H. Zhang. 2004b. Evaluation of behavioral factors influencing reproductive success and failure in captive giant pandas. Zoo Biology 23:15-31.

Zhang, H., D. Li, C. Wang, and V. Hull. 2009. Delayed implantation in giant pandas: the first comprehensive empirical evidence. Reproduction 138:979-986.

Zhang, Y. P., O. A. Ryder, Q. G. Zhao, Z. Y. Fan, G. X. He, A. J. Zhang, H. M. Zhang, T. M. He, and C. Yucun. 1994. Non-invasive giant panda paternity exclusion. Zoo Biology 13:569-573.

Zhi, L., P. Wenshi, Z. Xiaojian, W. Dajun, and W. Hao. 2000. What has the panda taught us? CONSERVATION BIOLOGY SERIES-CAMBRIDGE-:325-334. 


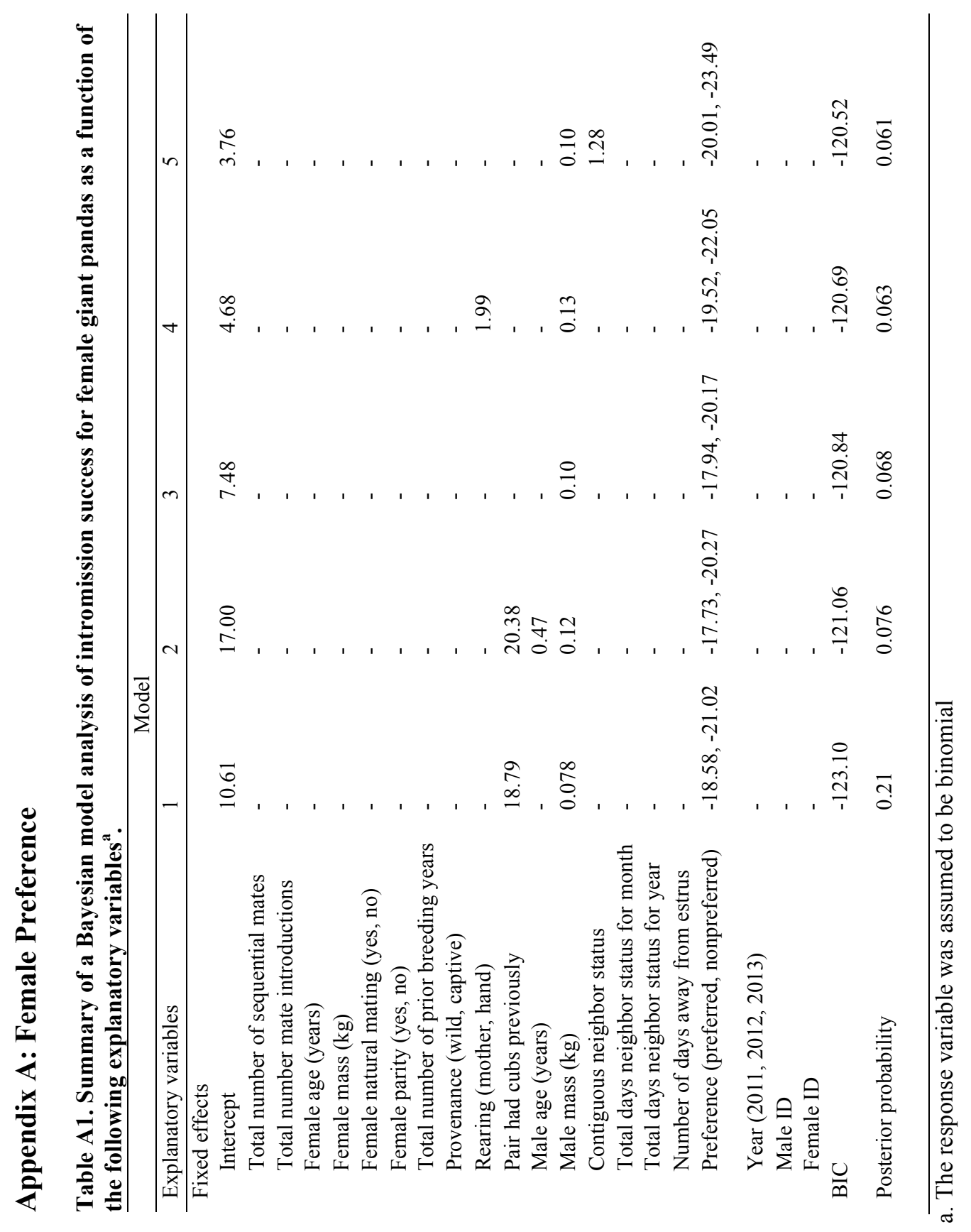




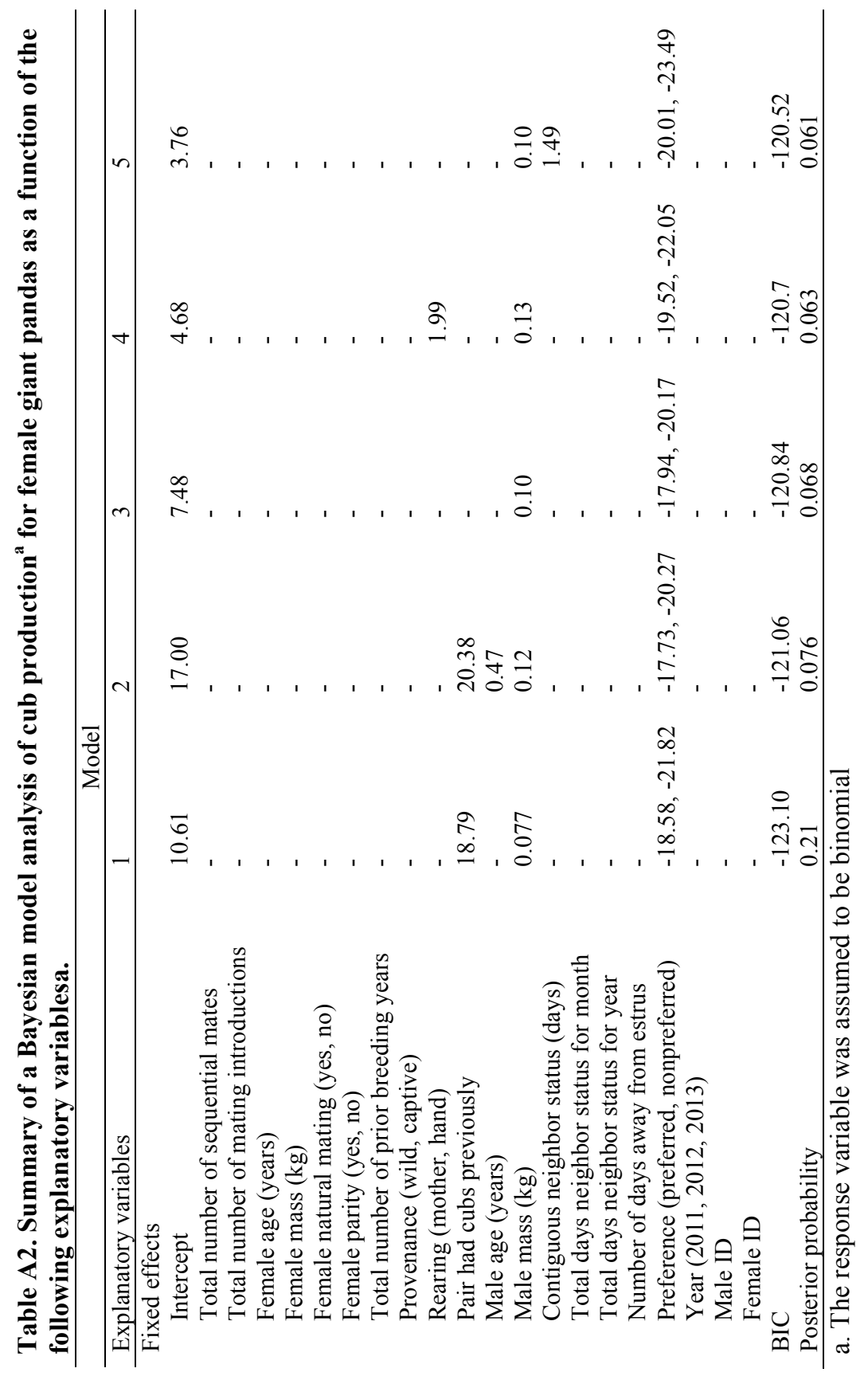




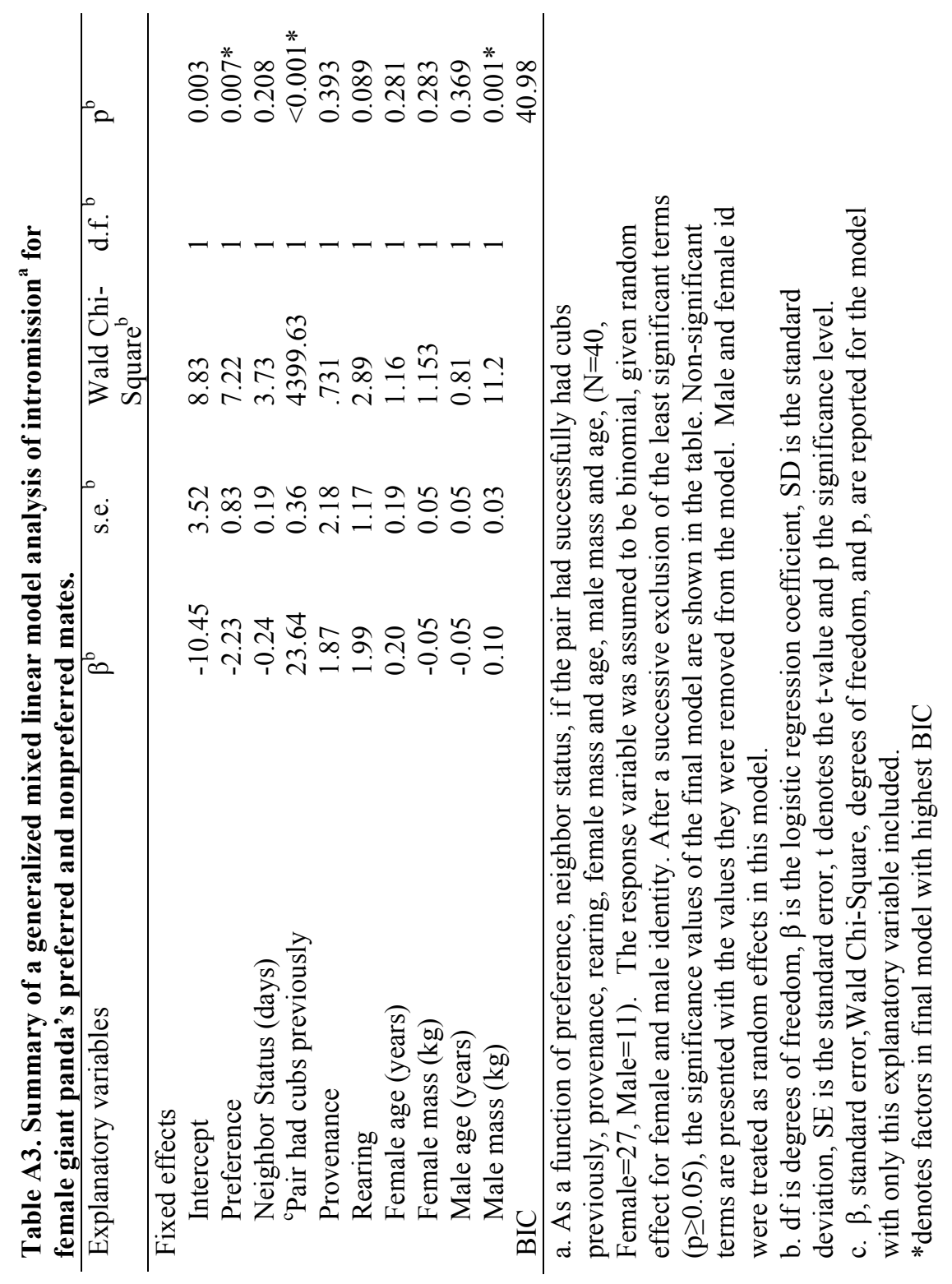




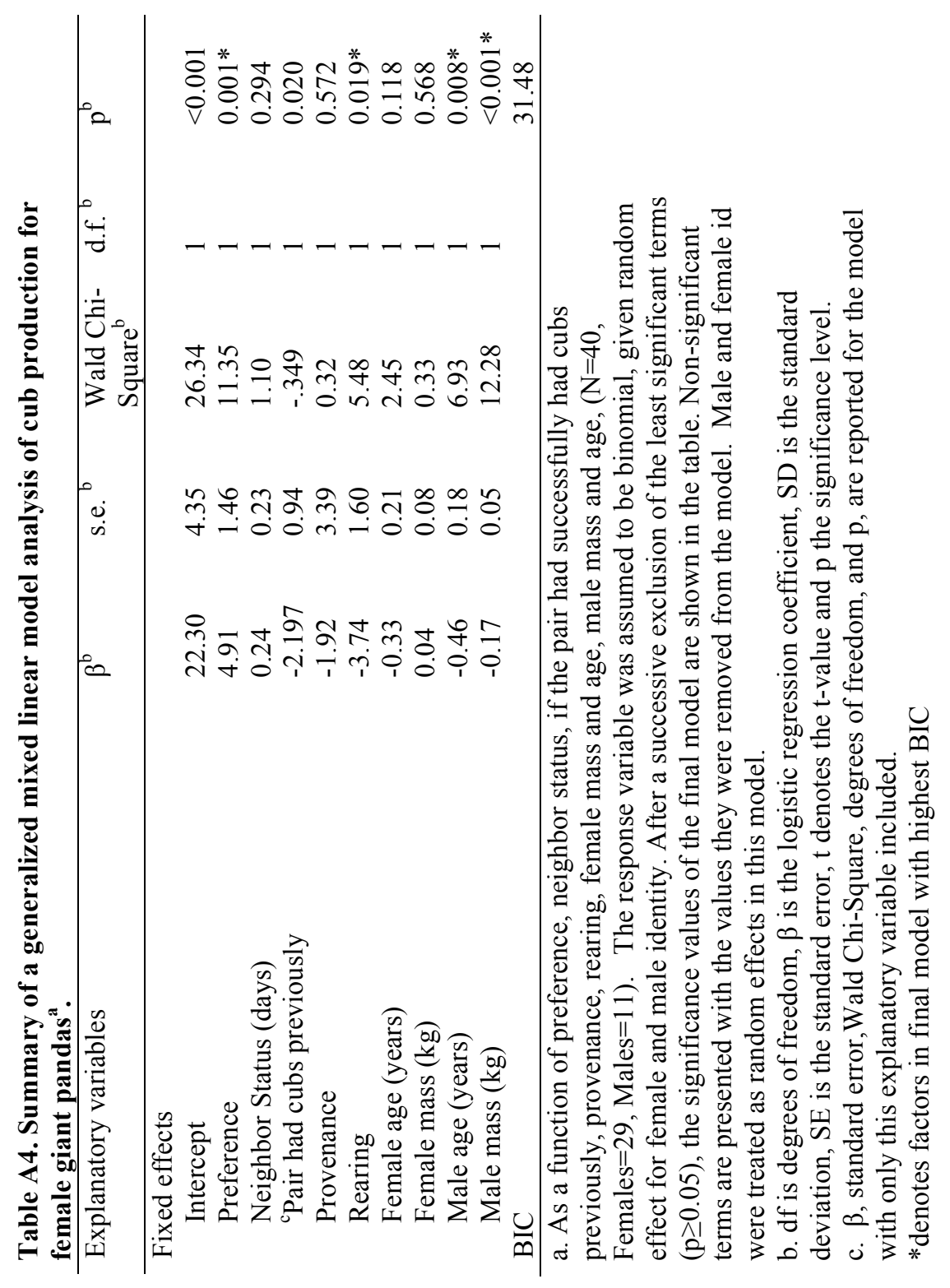




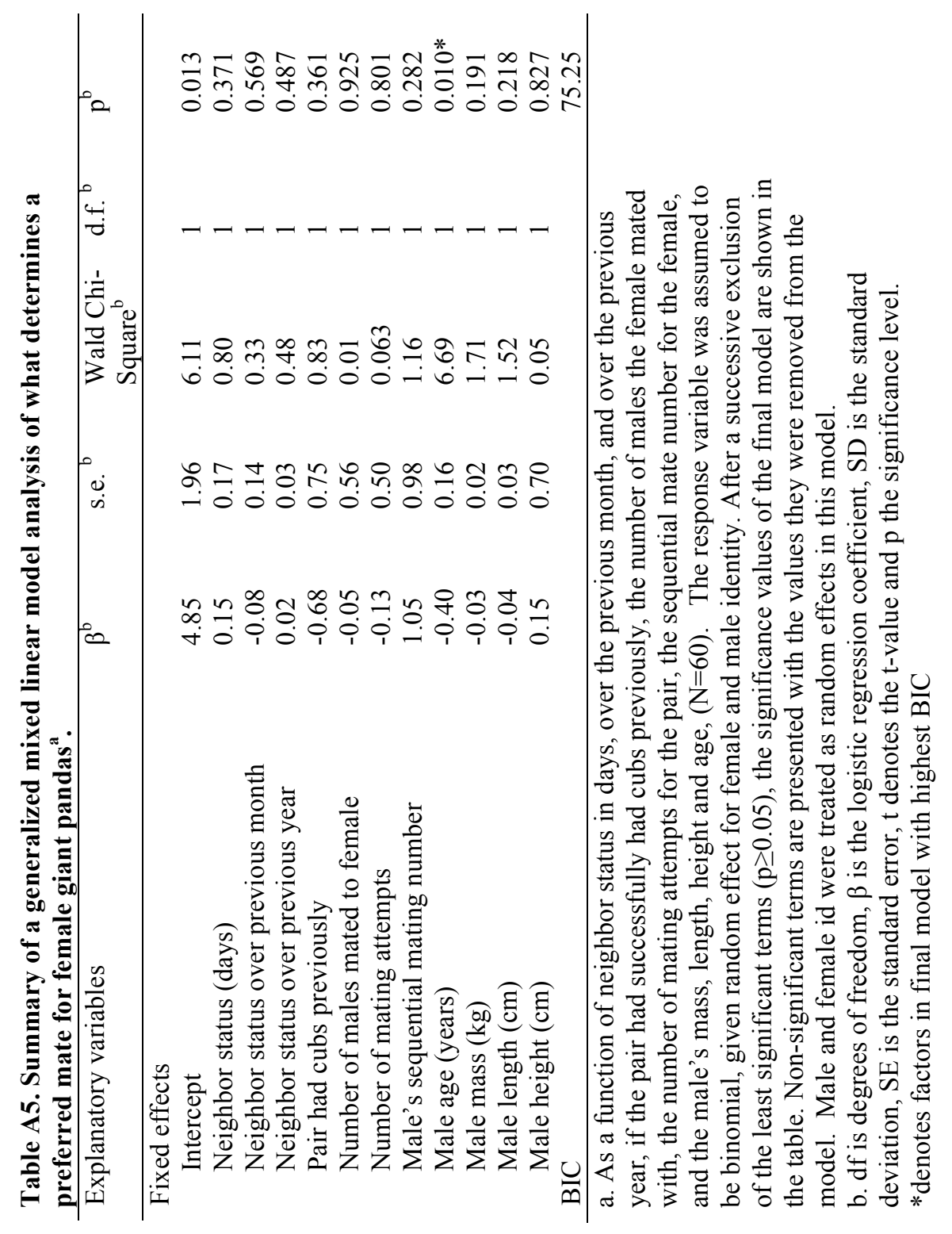




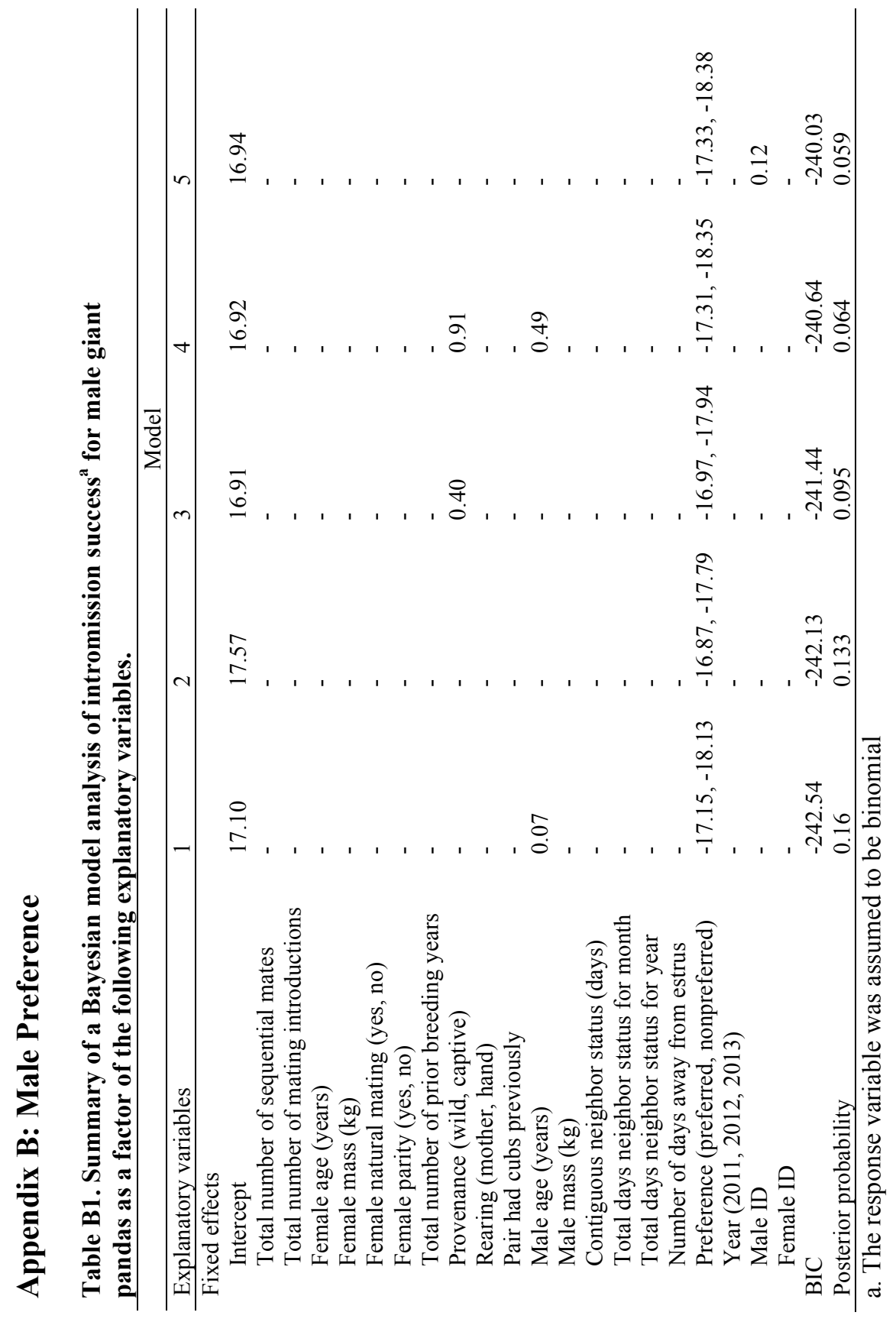




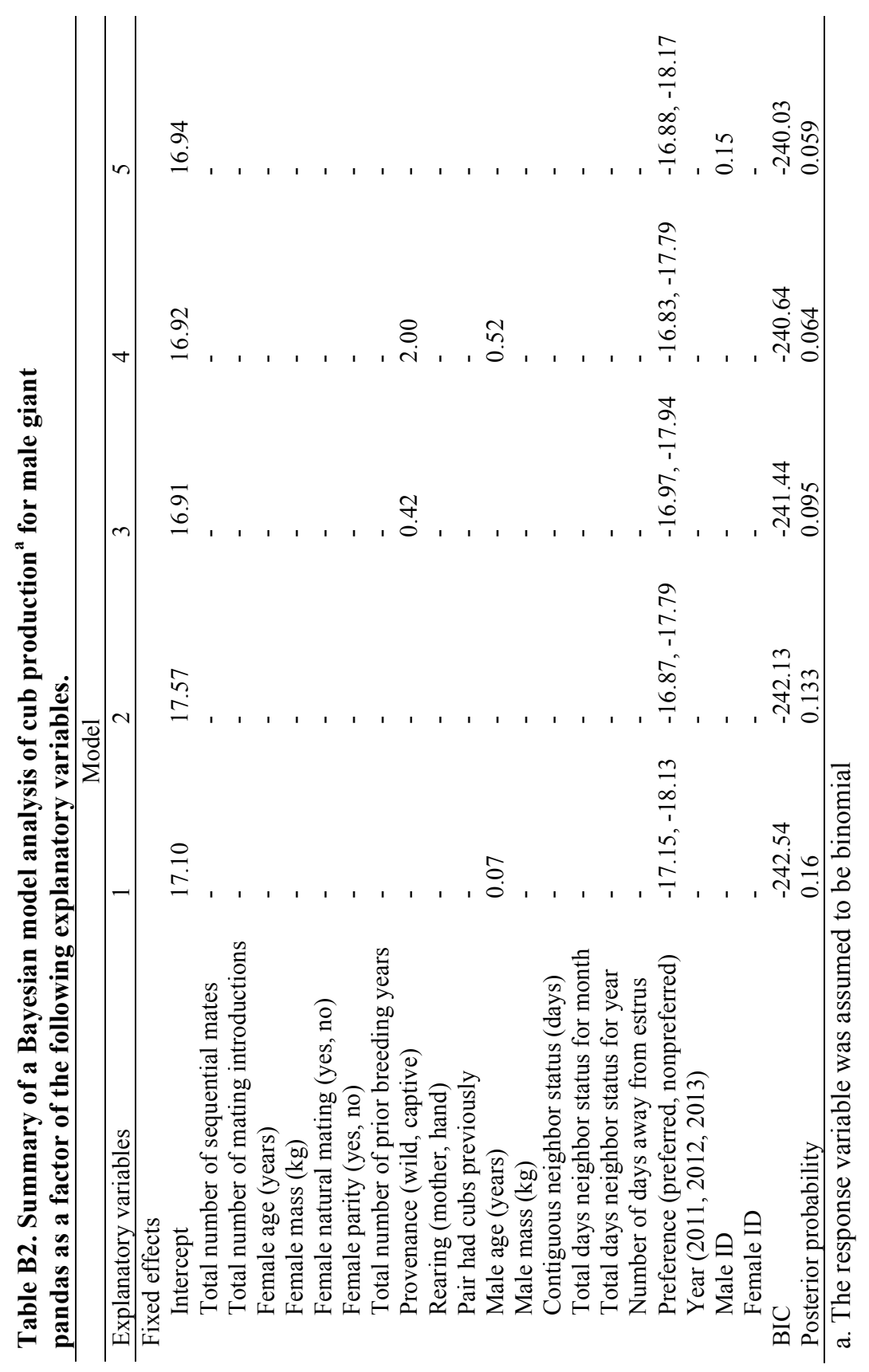




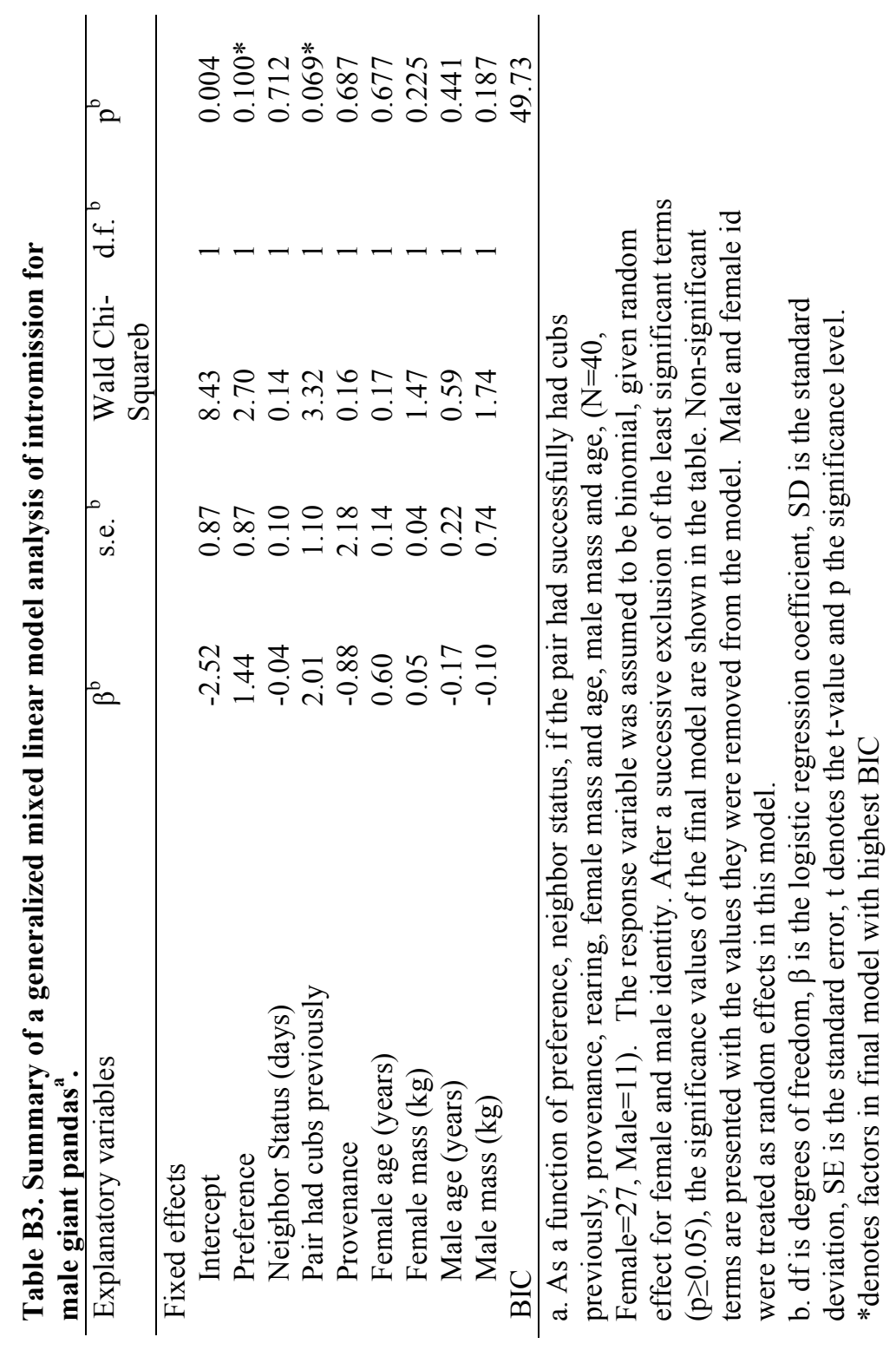




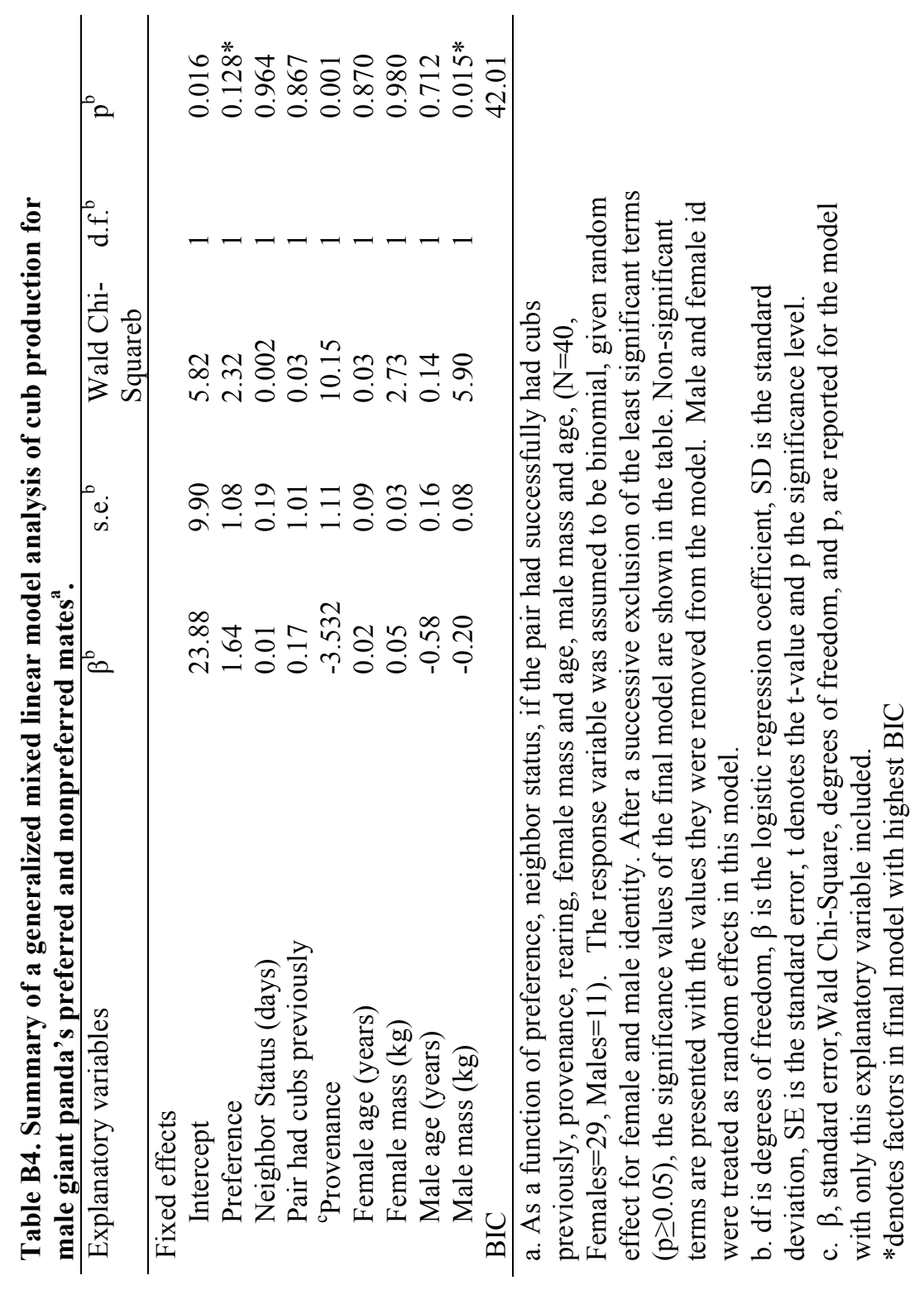




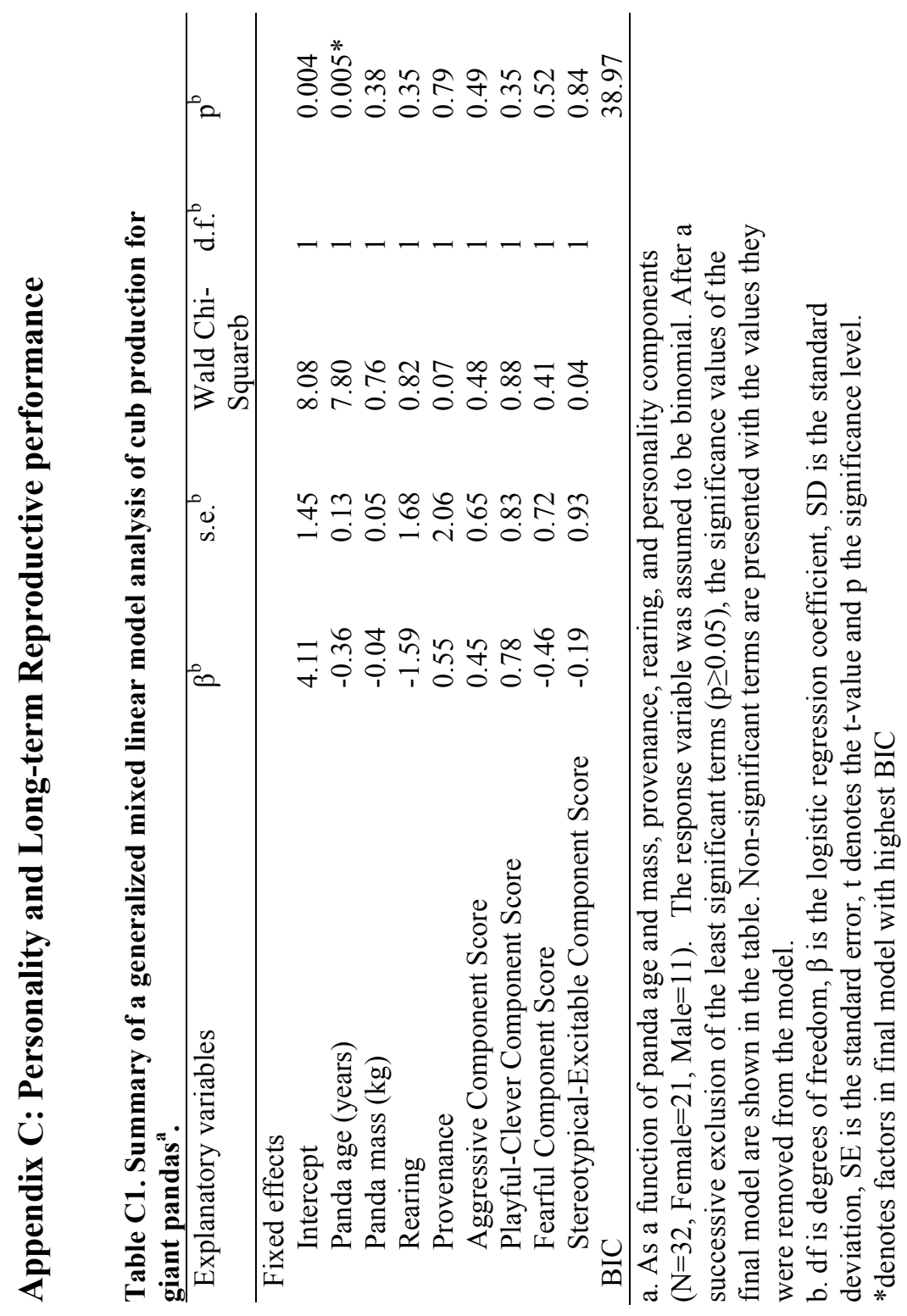




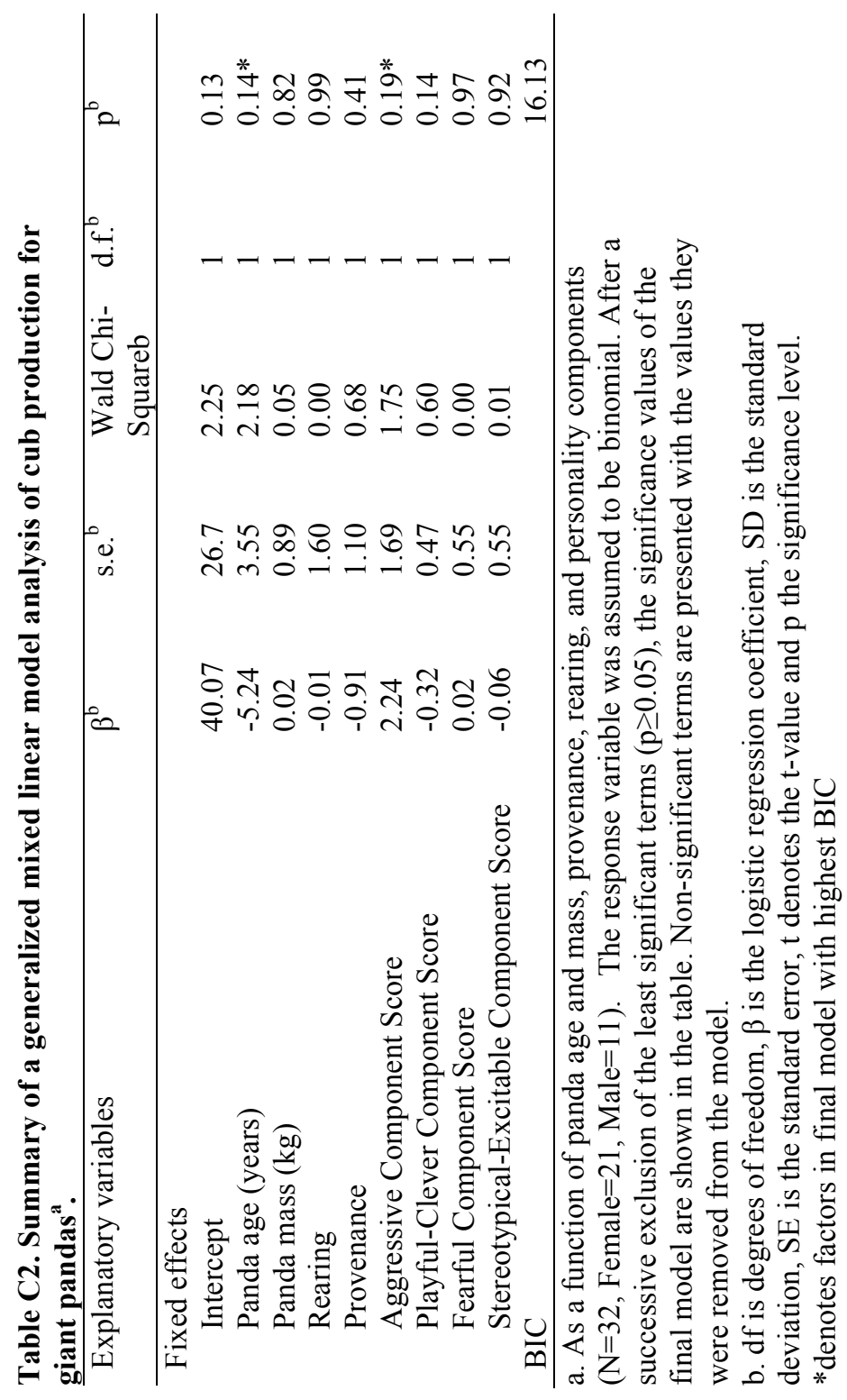




\section{Appendix D. Raw keeper personality scores and specific personality combinations within a mate dyad}

Mate dyads divided by 'active' keeper ratings were significantly different between groups in intromission success $\left(\chi^{2}(2)=6.09, \mathrm{p}=0.05\right)$ and cub production $\left(F_{2,27}=4.65, p=.02\right)$ but the H_L group had to be excluded due to inadequate sample sizes. Tukey HD post hoc tests indicated that $\mathrm{L}_{-} \mathrm{H}$ mate pairings on the keeper rating of 'active' had more successful intromissions and produced cubs more often. For intromission success, 'active' L_H dyads had marginally significantly more intromission success than L_L $(p=0.07)$ but not $\mathrm{H}_{-} \mathrm{H}(p=$ 0.38). For cub production, L_H mate dyads produced significantly more cubs than H-H $(p=0.03)$, but not L_L $(p=0.11)$.

The keeper rating 'aggressive toward conspecifics' positively loaded on the aggressive PCA component score as explained above. Whether a pair was compatible based on 'aggressive toward conspecifics' keeper ratings was significantly different between groups on intromission success $\left(\chi^{2}(3)=8.15, p=\right.$ $0.04)$ and in cub production $\left(\mathrm{F}_{3,25}=3.62, \mathrm{p}=.03\right)$. Tukey HD post hoc tests indicated that L_H mate pairings on the keeper rating of 'aggressive toward conspecifics' had more successful intromissions than L_L $(p=0.03)$ but not H_L mate dyads $(p=0.14)$ or H_H $(p=0.42)$. Tukey HD post hoc tests indicated that L_H mate pairings on the keeper rating 'aggressive toward conspecifics' had more successful cub production than L_L $(p=0.03)$ but not H_L mate dyads $(p=0.14)$ or H_H $(p=0.42)$.

Whether a pair was compatible based on 'calm' keeper ratings was significantly different between groups on intromission success and cub production. 
Sample sizes did not permit comparison between $\mathrm{H}_{-} \mathrm{L}$ and $\mathrm{H} \_\mathrm{H}$ groups.

Independent samples t-tests indicated that $\mathrm{L}_{-} \mathrm{L}$ mate pairings on the keeper rating of 'calm' had more successful intromissions than $\mathrm{L}_{-} \mathrm{H}(t(25)=2.68, \mathrm{p}=0.01)$. Independent samples t-tests indicated that $\mathrm{L}_{-} \mathrm{L}$ mate pairings on the keeper rating of 'aggressive toward conspecifics' had more successful cub production than $\mathrm{L}_{-} \mathrm{H}$ $(t(24)=3.19, \mathrm{p}=0.004)$

The keeper rating 'innovative' positively loaded on the playful PCA component score as explained above. Mate dyads divided by 'innovative' keeper ratings were significantly different between groups in intromission success $\left(\chi^{2}(3)=\right.$ $14.83, p=0.002)$ and cub production $\left(\chi^{2}(3)=8.70, \mathrm{p}=0.03\right)$. Tukey HD post hoc tests indicated that L_L dyads on 'innovative' had more successful intromissions and produced cubs more often. For intromission success, L_L dyads had significantly more intromission successes than $\mathrm{L}_{-} \mathrm{H}(p=0.002)$ and $\mathrm{H} \_\mathrm{H}(p=$ 0.003 ) dyads but not H_L dyads $(p=0.99)$. Further, post hoc tests indicated that L_H dyads had significantly less intromission success from $\mathrm{H} \_\mathrm{L}$ dyads $(p=0.01)$ but not $\mathrm{H} \_\mathrm{H}$ dyads $(p=0.92)$. H_L dyads had significantly more intromission success than H_H dyads $(p=0.02)$. Tukey HD post hoc tests indicated that for cub production, $\mathrm{L} \_\mathrm{H}$ mate dyads produced significantly more cubs than $\mathrm{H} \_\mathrm{L}(p=$ $0.03)$, but not L_L $(p=0.13)$ or $\mathrm{H}_{-} \mathrm{H}(p=0.10)$. 